NIST Handbook 152

\title{
Recommended Practice; Symbols, Terms, Units and Uncertainty Analysis for Radiometric Sensor Calibration
}

Clair L. Wyatt, Victor Privalsky, and Raju Datla

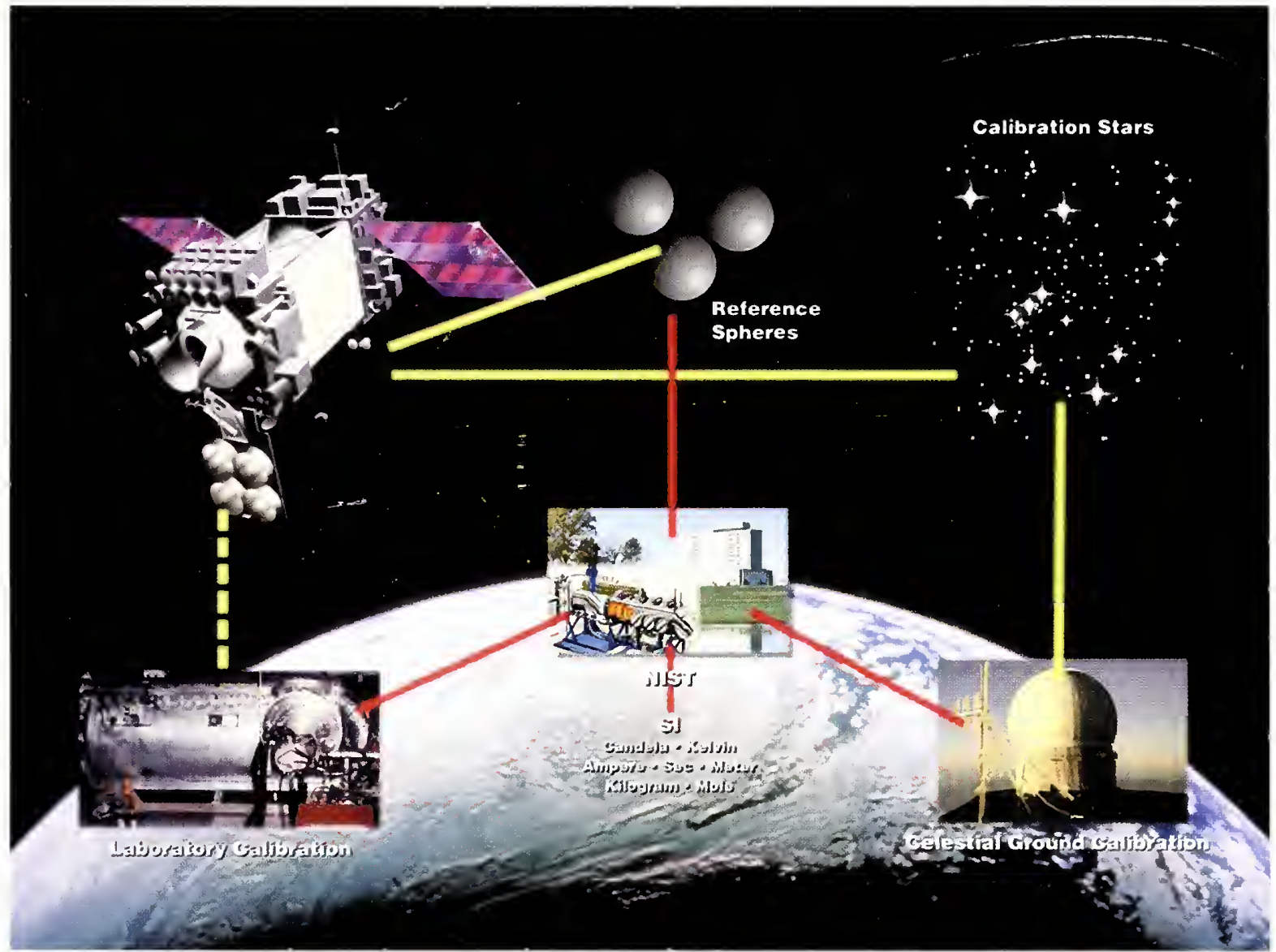

:partment of Commerce

4. logy Administration

1

il Institute of Standards and Technology 
he National Institute of Standards and Technology was established in 1988 by Congress to "assist industry in

the development of technology ... needed to improve product quality, to modernize manufacturing processes, to ensure product reliability ... and to facilitate rapid commercialization ... of products based on new scientific discoveries."

NIST, originally founded as the National Bureau of Standards in 1901, works to strengthen U.S. industry's competitiveness; advance science and engineering; and improve public health, safety, and the environment. One of the agency's basic functions is to develop, maintain, and retain custody of the national standards of measurement, and provide the means and methods for comparing standards used in science, engineering, manufacturing, commerce, industry, and education with the standards adopted or recognized by the Federal Government.

As an agency of the U.S. Commerce Department's Technology Administration, NIST conducts basic and applied research in the physical sciences and engineering, and develops measurement techniques, test methods, standards, and related services. The Institute does generic and precompetitive work on new and advanced technologies. NIST's research facilities are located at Gaithersburg, MD 20899, and at Boulder, CO 80303. Major technical operating units and their principal activities are listed below. For more information contact the Publications and Program Inquiries Desk, 301-975-3058.

\section{Office of the Director}

- National Quality Program

- International and Academic Affairs

Technology Services

- Standards Services

- Technology Partnerships

- Measurement Services

- Technology Innovation

- Information Services

\section{Advanced Technology Program}

- Economic Assessment

- Information Technology and Applications

- Chemical and Biomedical Technology

- Materials and Manufacturing Technology

- Electronics and Photonics Technology

\section{Manufacturing Extension Partnership}

Program

- Regional Programs

- National Programs

- Program Development

\section{Electronics and Electrical Engineering} Laboratory

- Microelectronics

- Law Enforcement Standards

- Electricity

- Semiconductor Electronics

- Electromagnetic Fields'

- Electromagnetic Technology'

- Optoelectronics ${ }^{1}$

\section{Chemical Science and Technology}

Laboratory

- Biotechnology

- Physical and Chemical Properties ${ }^{2}$

- Analytical Chemistry

- Process Measurements

- Surface and Microanalysis Science
Physics Laboratory

- Electron and Optical Physics

- Atomic Physics

- Optical Technology

- Ionizing Radiation

- Time and Frequency ${ }^{1}$

- Quantum Physics'

\section{Materials Science and Engineering} Laboratory

- Intelligent Processing of Materials

- Ceramics

- Materials Reliability

- Polymers

- Metallurgy

- NIST Center for Neutron Research

\section{Manufacturing Engineering}

Laboratory

- Precision Engineering

- Automated Production Technology

- Intelligent Systems

- Fabrication Technology

- Manufacturing Systems Integration

\section{Building and Fire Research}

Laboratory

- Structures

- Building Materials

- Building Environment

- Fire Safety Engineering

- Fire Science

\section{Information Technology Laboratory}

- Mathematical and Computational Sciences ${ }^{2}$

- Advanced Network Technologies

- Computer Security

- Information Access and User Interfaces

- High Performance Systems and Services

- Distributed Computing and Information Services

- Software Diagnostics and Conformance Testing

\footnotetext{
'At Boulder, $\mathrm{CO} 80303$.

${ }^{2}$ Some elements at Boulder, CO.
} 


\section{Recommended Practice; Symbols, Terms, Units and Uncertainty Analysis for Radiometric Sensor Calibration}

Dr. Clair L. Wyatt, Professor Emeritus

Electrical Engineering Department

and

Dr. Victor Privalsky, Sr. Scientist

Space Dynamics Laboratory

Utah State University

and

Dr. Raju Datla

Optical Technology Division

Physics Laboratory

National Institute of Standards and Technology

Gaithersburg, MD 20899-0001

September 1998

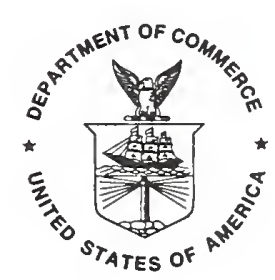

U.S. DEPARTMENT OF COMMERCE, William M. Daley, Secretary

Technology Administration, Gary R. Bachula, Acting Under Secretary for Technology National Institute of Standards and Technology, Raymond G. Kammer, Director 
National Institute of Standards and Technology Handbook 152

Natl. Inst. Stand. Technol. Handb. 152, 120 pages (Sept. 1998)

CODEN: NIHAE2

U.S. GOVERNMENT PRINTING OFFICE

WASHINGTON: 1998

For sale by the Superintendent of Documents, U.S. Government Printing Office, Washington, DC 20402-9325 


\section{FOREWARD}

Uniform terminology and common practices of uncertainty analysis are absolutely crucial for the ground based or space based radiometry projects of the National Aeronautics and Space Administration (NASA), the National Oceanographic and Atmospheric Administration (NOAA) and the Department of Defense (DoD) to exchange scientific data and results without the need for duplication and repetition. The economic impact is even greater for exchanging data and results around the world on global studies which is only possible through uniformity of terminology and data analysis standards.

This need for developing a common practice for quantities, symbols, units and uncertainty analysis has been recognized by scientists and engineers around the world. The first step taken to my knowledge recently was the establishment of Space Based Observation Systems Committee on Standards (SBOS COS) in 1988 by the American Institute of Aeronautics and Astronautics (AIAA). As a historical perspective, the letter by Christopher Stevens of Jet Propulsion Laboratory that shows various meetings in this endeavor and the overview on "AIAA activities in Calibration Standards" by Edward Koenig of ITT Aerospace/ Communications Division is reproduced in Appendix 1. It also lists the members of the subcommittee on sensor systems. I would like to join with Clair Wyatt, the principal author of this document, in acknowledging the efforts of various people in that list who helped in preparing this document. It is being published as a NIST Handbook recommending it to be a common practice for optical radiation metrology. It primarily deals with terms, symbols and definitions in radiometry based on the International Standards Organization (ISO) definitions of basic radiometric quantities. The sensor systems calibration methodology is based on the measurement equation approach that has been in practice from the beginning at the National Institute of Standards and Technology (NIST). The uncertainty analysis is based on the ISO Guide to the Expression of Uncertainty in Measurement, ISO/TAG $4 W G 3$.

\section{Raju Datla, Optical Technology Division, NIST}




\section{PREFACE}

This recommended practice introduces several new entities. Of concern are the terms, symbols, and units (nomenclature) used to describe sources, sensor performance analysis, calibration, and uncertainty analysis of radiometric sensors. The definitions given in this document are limited to those that apply to radiometric calibration and do not include illumination terms. It has been the authors' dream to create a document like this to facilitate communication and dissemination of knowledge throughout the optical community. It is heartening to note that one of the authors, Dr. V. Privalsky was already chosen by the Russian Space Agency to translate this document into Russian.

The contents of this document were presented as a tutorial at the Fifth Infrared Radiometric Sensor Calibration Symposium that was held by Space Dynamics Laboratory /Utah State University in Logan, Utah, in May 1995. The document was revised based on the comments of the participants to its present form.

Authors.

Key Words: Radiometry, Sensor Calibration, Uncertainty Analysis 
TABLE OF CONTENTS

FORWARD

PREFACE

INTRODUCTION

1. PART 1: SYMBOLS, TERMS, AND UNITS 2

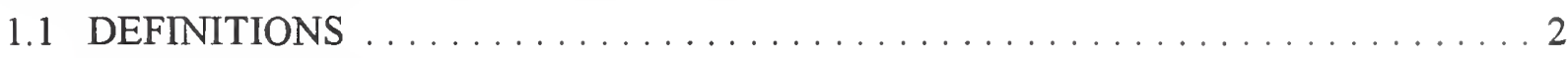

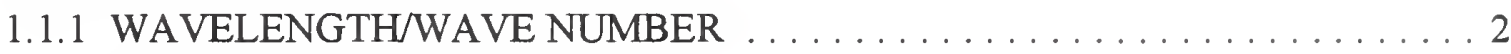

1.1 .2 FLUX . . . . . . . . . . . . . . . . . . . . . . . . 3

1.2 GEOMETRICAL PROPERTIES OF SOURCES $\ldots \ldots \ldots \ldots \ldots \ldots \ldots \ldots$

1.2.1 RADIANCE/PHOTON RADIANCE .................... 3

1.2.2 RADIANT EXITANCE/PHOTON EXITANCE . . . . . . . . . . . . . 4

1.2.3 RADIANT INTENSITY/PHOTON INTENSITY . . . . . . . . . . . . 4

1.2.4 IRRADIANCE/PHOTON IRRADIANCE . . . . . . . . . . . . . 6

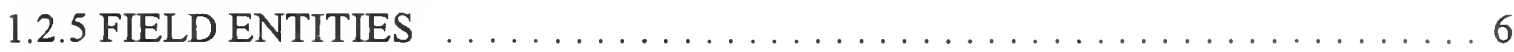

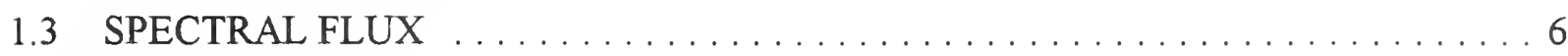

1.4 THE GEOMETRY OF RADIATION TRANSFER $\ldots \ldots \ldots \ldots \ldots \ldots \ldots \ldots$

1.4.1 PROJECTED AREA . . . . . . . . . . . . . . . . . . . . . . . 7

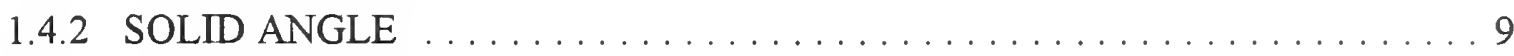

1.4 .3 PROJECTED SOLID ANGLE $\ldots \ldots \ldots \ldots \ldots \ldots \ldots \ldots \ldots \ldots \ldots \ldots \ldots \ldots \ldots$

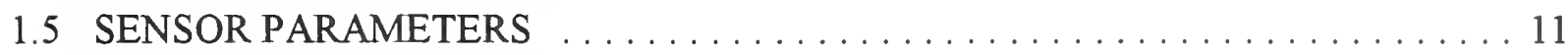

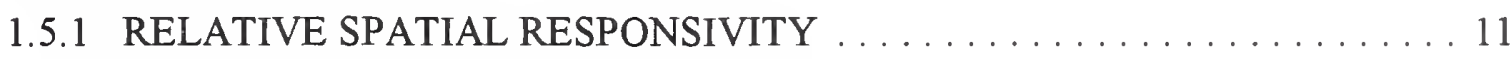

1.5.2 ENCIRCLED (ENSQUARED) ENERGY . . . . . . . . . . . . 12

1.5.3 THROUGHPUT AND RELATIVE APERTURE . . . . . . . . . . 12

2. PART II: THE RADIOMETRIC SENSOR CALIBRATION $\ldots \ldots \ldots \ldots \ldots \ldots \ldots$

2.1 THE MEASUREMENT EQUATION . . . . . . . . . . . . . . . . . . . 15 


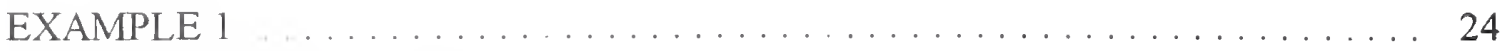

2.2 CALIBRATION EQUATIONS ........................... 26

2.2.1 RADIOMETER RADIANCE CALIBRATION EQUATION . . . . . . . . . 28

2.2.2 RADIOMETER IRRADIANCE CALIBRATION EQUATION . . . . . . . . . 30

2.2.3 SPECTROMETER CALIBRATION EQUATION . . . . . . . . . . . 31

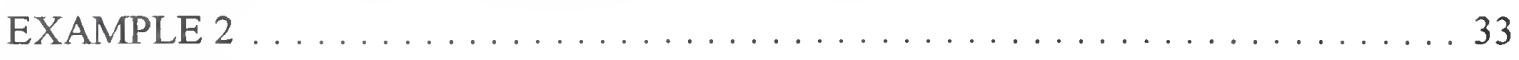

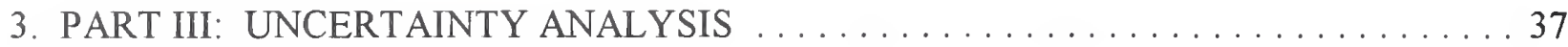

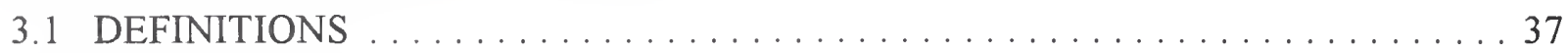

3.2 UNCERTAINTY ANALYSIS FOR SENSOR CALIBRATION . . . . . . . . . . . 39

3.2 .1 CALIBRATION SNAPSHOTS ......................... 39

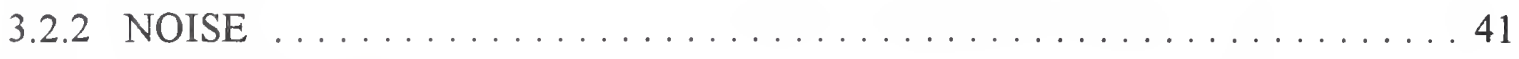

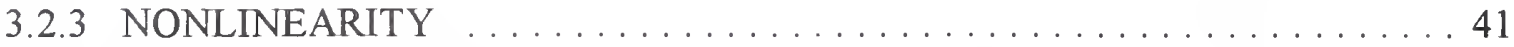

3.2 .4 NONUNIFORM AREA RESPONSIVITY . . . . . . . . . . . . . . . 44

3.2 .5 ANGULAR SPATIAL RESPONSIVITY . . . . . . . . . . . . . . . . . . 46

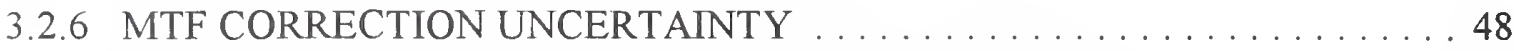

3.2.7 CALIBRATION STANDARD SOURCE UNCERTAINTY . . . . . . . . . 49

3.2 .8 ABSOLUTE RESPONSIVITY UNCERTAINTY . . . . . . . . . . . 50

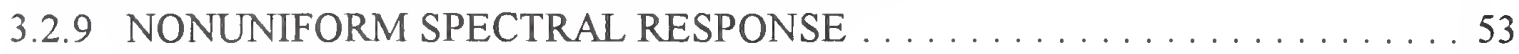

3.2 .10 BAND-TO-BAND UNCERTAINTY . . . . . . . . . . . . . . . 57

3.3 PROPAGATION OF UNCERTAINTIES - COMBINED STANDARD

UNCERTAINTY . . . . . . . . . . . . . . . . . . . . . . 58

3.3.1 OLD TERMINOLOGY AND RECOMMENDED PRACTICE . . . . . . . . 59

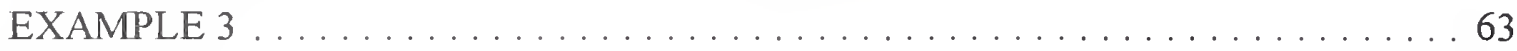

4. REFERENCES ................................... 73 


\section{TABLES}

TABLE 1. BASIC RADIOMETRIC TERMS, SYMBOLS, AND UNITS $\ldots \ldots \ldots \ldots .5$

TABLE 2. SOURCE SPECTRAL TERMS, SYMBOLS, AND UNITS $\ldots \ldots \ldots \ldots \ldots . .$.

TABLE 3. GONIOMETRIC TERMS AND UNITS . . . . . . . . . . . . . . . 14

TABLE 4. SYSTEM PERFORMANCE TERMS, SYMBOLS, AND UNITS . . . . . . 22

TABLE 5. SYSTEM CALIBRATION TERMS, SYMBOLS, AND UNITS . . . . . . . . . 29

TABLE 6. UNCERTAINTY ANALYSIS SYMBOLS AND TERMS . . . . . . . . 40

FIGURES

FIGURE 1. ILLUSTRATION OF THE PROJECTED AREA $\ldots \ldots \ldots \ldots \ldots \ldots$

FIGURE 2. ILLUSTRATION OF SOLID ANGLE AND PROJECTED SOLID ANGLE . 10 FIGURE 3. SCHEMATIC FOR A SIMPLE OPTICAL SYSTEM ILLUSTRATING THE HALF-ANGLE FIELD-OF-VIEW $\Theta$, AND THE CONE HALF-ANGLE $\alpha \ldots 13$

FIGURE 4. RELATIVE RESPONSE AND SPECTRAL RADIANCE OF A FILTER RADIOMETER

FIGURE 5. ILLUSTRATION OF DATA LINEARIZATION. THE ORIGINAL DATACIRCLE, LINEARIZED DATA-SQUARE, SOLID CURVE IS THE IDEAL LINEAR RESPONSE.

FIGURE 6. ISOMETRIC 3-DIMENSIONAL VIEW OF THE SPATIAL RESONSE OF AN INFRARED DETECTOR.

FIGURE 7. POINT (---) AND LINE-SPREAD (---) FUNCTION MTF FOR

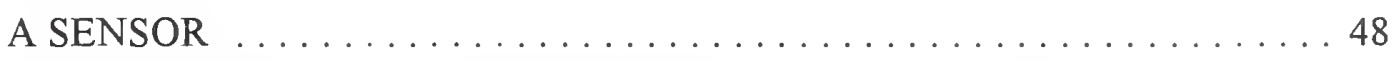

FIGURE 8. TYPICAL BANDPASS INTERFERENCE FILTER TRANSMITTANCE . . . 55

FIGURE 9. STANDARD UNCERTAINTY (1-SIGMA GIVEN IN PERCENT) OF THE EXTENDED- AREA SOURCE ABSOLUTE RESPONSIVITY CALIBRATION . 
FIGURE 10. RELATIVE SPECTRAL RESPONSE $\rho_{\mathrm{L}}(\lambda)$ FOR THE EXTENDED-AREA SOURCE ABSOLUTE CALIBRATION . . . . . . . . . . . . 66

FIGURE 11. SCANS OBTAINED FOR THE POINT-SOURCE ABSOLUTE IRRADIANCE RESPONSIVITY CALIBRATION ..................66

FIGURE 12. STANDARD UNCERTAINTY (1- SIGMA) OF THE POINT-SOURCE ABSOLUTE IRRADIANCE RESPONSIVITY CALIBRATION . . . . . . . . . 67

FIGURE 13. RELATIVE SPECTRAL RESPONSE $\rho_{\mathrm{L}}(\lambda)$ FOR THE POINT-SOURCE ABSOLUTE CALIBRATION

FIGURE 14. SCANS OBTAINED FOR THE EXTENDED-AREA SOURCE ABSOLUTE RADIANCE RESPONSIVITY CALIBRATION

\section{APPENDIX}

APPENDIX 1 . 


\section{INTRODUCTION}

This handbook provides recommendations for nomenclature, terms, symbols, units and uncertainty analysis associated with the calibration of radiometric sensor systems. The scope includes the radiant properties of sources; the geometry of radiation transfer; the measurement equation used to predict sensor response; the calibration equation used to convert sensor response to engineering units (radiance, irradiance, etc.); and the uncertainty analysis.

The contents are organized to correspond, somewhat, to the normal flow of flux (source to sensor) and of analysis (predicted performance to generation of calibration equations and uncertainty analysis). This document expands on the current practice of radiometry as described in a recent NIST Technical Note [1].

The definitions of radiometric terms, symbols, and units in this document conform to the definitions accepted by the International Standards Organizations (ISO)[2]. These standards include quantities that are functions of wavelength (frequency or wavenumber); they may be designated by the same term preceded by the adjective spectral and by the same symbol followed by $\lambda$, $v$, or $\sigma$ in parenthesis; for example spectral emissivity $\epsilon(\lambda)$. On the other hand, if the spectral power density, or spectral power concentration [3] is considered, it may also be designated by the name of the quantity and by the symbol for the quantity with the subscript $\lambda$ ( $v$, or $\sigma$ ); for example the spectral radiance,

$$
L_{\lambda}=\frac{\mathrm{d} L}{\mathrm{~d} \lambda} .
$$

Note that $L_{\lambda}\left[\mathrm{W} /\left(\mathrm{m}^{3} \mathrm{sr}\right)\right]$ corresponds to watts per unit area per unit wavelength $\left[\left(\mathrm{W} /\left(\mathrm{m}^{2} \mathrm{sr}\right)\right) / \mu \mathrm{m}\right]$ rather than watts per unit volume. Generally, wavelength is expressed in micrometers $(\mu \mathrm{m})$ for infrared and in nanometers ( $\mathrm{nm}$ ) for ultraviolet and visible regions of the spectrum. The integrated quantity is given by

$$
L=\int L_{\lambda} \mathrm{d} \lambda
$$

with units $\left[\mathrm{W} /\left(\mathrm{m}^{2} \mathrm{sr}\right)\right]$. In this document the NIST Guide for the Use of the International System 
of Units (SI) is followed [4]. Also, the SI base units for quantities are in square brackets when they are introduced for the first time.

The terms used for uncertainty analysis conform to the ISO Guide to the Expression of Uncertainty in Measurement [5]. Based on the ISO Guide, NIST developed the guidelines for uncertainty analysis. The document describing these guidelines is added as Appendix 2 [6].

Those aspects of the ISO Guide that impinge upon this document are as follows. The standard uncertainty refers to components of uncertainty including both random and systematic effects. Note that the term random is used rather than the term "precision," and that the term systematic is used rather than the term "bias." The term combined standard uncertainty is used rather than the term "accuracy" and has reference to propagated uncertainties. Finally, the term uncertainty analysis is used rather than the term "error analysis."

\section{PART 1: SYMBOLS, TERMS, AND UNITS}

\subsection{DEFINITIONS}

As indicated above, the scope of this document is limited to those symbols, terms, and units frequently used in the calibration of radiometric and spectrometric systems. Consequently, there is no attempt to create an exhaustive list of terms.

In order to avoid large or small numerical values, decimal multiples and sub-multiples of the SI units are added to the system making use of the standard prefixes [7]; for example, centimeter with a factor of $10^{-2}$ and a symbol of $\mathrm{cm}$, nanometer with a factor of $10^{-9}$ and a symbol of $\mathrm{nm}$, and micrometer with a factor of $10^{-6}$ and a symbol of $\mu \mathrm{m}$.

The ISO standard also addresses the question of alternative names and symbols for various terms. It also recognizes a class of "supplementary" units like the radian and steradian as a class of dimensionless units [8].

\subsubsection{WAVELENGTH/WAVENUMBER}

The wavelength $\lambda[\mathrm{m}]$ is defined as the distance between two adjacent points in a periodic wave having the same phase. The wavenumber $\sigma\left[\mathrm{m}^{-1}\right]$ is the number of waves in a given length interval. 


\subsubsection{FLUX}

The radiant energy flux $\Phi_{\mathrm{e}}[\mathrm{J} / \mathrm{s}$ or $\mathrm{W}]$ is the power emitted, transferred or received; $\Phi_{\mathrm{p}}\left[\mathrm{s}^{-1}\right]$ is the quanta-rate emitted, transferred or received. The subscripts e and $p$ refer to energy and photon rates respectively. The symbol $\Phi$ is used without subscripts when it is clear from the context.

\subsection{GEOMETRICAL PROPERTIES OF SOURCES}

Sources are characterized in terms of geometrical properties to facilitate calculations using the geometry of radiation transfer [9]. Table 1 provides a list of terms, units, and symbols for characterizing sources. Also indicated in the table are the types of geometrical information inherent in the entity: positional and/or directional. Definitions are given, in the sections to follow, for each of the source characterizations listed in Table 1.

\subsubsection{RADIANCE/PHOTON RADIANCE}

The average radiance $L_{\text {ave }}$ of a source is the ratio of the total flux [W] to the product of the projected source area $A_{S} \cos \theta$ and the solid angle $\omega_{S}$ into which the radiation is emitted. The subscript $s$ refers to the source. This definition also holds for average photon radiance except the total flux has units of photons per unit time $\left[\mathrm{s}^{-1}\right]$. The radiance $L$ at a point on the source in a certain direction is given by

$$
L=\lim _{\Delta A_{\mathrm{s}}, \Delta \omega_{\mathrm{s}} \rightarrow 0}\left[\frac{\Delta \Phi}{\Delta A_{\mathrm{s}} \cos \theta \Delta \omega_{\mathrm{s}}}\right]=\frac{\mathrm{d}^{2} \Phi}{\mathrm{d} A_{\mathrm{s}} \cos \theta \mathrm{d} \omega_{\mathrm{s}}}
$$

The radiance is a measure of the flux of a source per unit area per unit solid angle at a point and in the direction of propagation. Thus the radiance provides the most general description of the source since it contains both positional and directional information. The total flux is given by

$$
\Phi=\iint L \cos \theta \mathrm{d} \omega_{\mathrm{s}} \mathrm{d} A_{\mathrm{s}}
$$




\subsubsection{RADIANT EXITANCE/PHOTON EXITANCE}

The average radiant exitance $M_{\text {ave }}$ of a source is the ratio of the total flux [W] to the total area of the source $A_{\mathrm{s}}$. This definition also holds for average photon exitance except the total flux has units of photons per unit time $\left[\mathrm{s}^{-1}\right]$. The limiting value of the average exitance of a small portion of the source as the area is reduced to a point is the radiant exitance $M$ of the source at a point and is given by

$$
M=\lim _{\Delta A_{\mathrm{s}} \rightarrow 0}\left[\frac{\Delta \Phi}{\Delta A_{\mathrm{s}}}\right]=\frac{\mathrm{d} \Phi}{\mathrm{d} A_{\mathrm{s}}}
$$

The radiant exitance is a positional measure of the emitted flux of a source per unit area at a point. The total flux is given by

$$
\Phi=\int M \mathrm{~d} A_{\mathrm{s}}
$$

\subsubsection{RADIANT INTENSITY/PHOTON INTENSITY}

The average radiant intensity $I_{\text {ave }}$ is the ratio of the total flux $[\mathrm{W}]$ to the total solid angle $\omega_{S}$ about the source. This definition also holds for average photon intensity except the total flux has units of photons per unit time $\left[\mathrm{s}^{-1}\right]$. For an isotropic source the flux is radiated into $4 \pi \mathrm{sr}$ (a sphere) and for a flat surface into $2 \pi \mathrm{sr}$ (a hemisphere). The limiting value of the average radiant intensity as the solid angle is reduced in value about a particular direction is the radiant intensity $I$ in that direction and is given by

$$
I=\lim _{\Delta \omega_{\mathrm{s}} \rightarrow 0}\left[\frac{\Delta \Phi}{\Delta \omega_{\mathrm{s}}}\right]=\frac{\mathrm{d} \Phi}{\mathrm{d} \omega_{\mathrm{s}}}
$$

The total flux is given by

$$
\Phi=\int I \mathrm{~d} \omega_{\mathrm{s}}
$$


TABLE 1

BASIC RADIOMETRIC TERMS, SYMBOLS, AND UNITS

(With geometrical information where appropriate)

\begin{tabular}{lll}
\hline Wavelength & $\lambda$ & {$[\mathrm{m}]$} \\
Wavenumber & $\sigma$ & {$\left[\mathrm{m}^{-1}\right]$} \\
& & \\
Radiant energy flux, Radiant power & $\Phi, \Phi_{\mathrm{e}}, P$ & {$[\mathrm{~W}]$ or $[\mathrm{J} / \mathrm{s}]$} \\
Photon flux & $\Phi_{\mathrm{p}}, \Phi$ & {$\left[\mathrm{s}^{-1}\right]$} \\
& & \\
Radiance (positional-directional) & $L, L_{\mathrm{e}}$ & {$\left[\mathrm{W} /\left(\mathrm{m}^{2} \mathrm{sr}\right)\right]$} \\
Photon radiance (positional- directional) & $L_{\mathrm{p}}, L$ & {$\left[\mathrm{~s}^{-1} /\left(\mathrm{m}^{2} \mathrm{sr}\right)\right]$ or } \\
& & {$\left[\mathrm{s}^{-1} \mathrm{~m}^{-2} \mathrm{sr}{ }^{-1}\right]$} \\
& & \\
Radiant exitance (positional) & $M, M_{\mathrm{e}}$ & {$\left[\mathrm{W} / \mathrm{m}^{2}\right]$} \\
Photon exitance (positional) & $M_{\mathrm{p}}, M$ & {$\left[\mathrm{~s}^{-1} / \mathrm{m}^{2}\right]$ or $\left[\mathrm{s}^{-1} \mathrm{~m}^{-2}\right]$} \\
& & \\
Irradiance (positional) & $E, E_{\mathrm{e}}$ & {$\left[\mathrm{W} / \mathrm{m}^{2}\right]$} \\
Photon irradiance (positional) & $E_{\mathrm{p}}, E$ & {$\left[\mathrm{~s}^{-1} / \mathrm{m}^{2}\right]$ or $\left[\mathrm{s}^{-1} \mathrm{~m}^{-2}\right]$} \\
& & \\
Radiant intensity (directional) & $I, I_{\mathrm{e}}$ & {$[\mathrm{W} / \mathrm{sr}]$} \\
Photon intensity (directional) & $I_{\mathrm{p}}, I$ & {$\left[\mathrm{~s}^{-1} / \mathrm{sr}\right]$ or $\left[\mathrm{s}^{-1} \mathrm{sr}^{-1}\right]$} \\
\hline
\end{tabular}

Note: Subscripts $e$ and $p$ as are not used when it is clear from the context. 


\subsubsection{IRRADIANCE/PHOTON IRRADIANCE}

The average irradiance $E_{\text {ave }}$ is the ratio of the total flux [W] to the total incident surface area, and is a measure of the incident flux per unit area. This definition also holds for average photon irradiance except the total flux has units of photons per unit time $\left[\mathrm{s}^{-1}\right]$. The limiting value of the average irradiance of a small portion of the incident surface $A_{\mathrm{c}}$ as the area is reduced to a point is the irradiance $E$ at that point is given by

$$
E=\lim _{\Delta A_{\mathrm{c}} \rightarrow 0}\left[\frac{\Delta \Phi}{\Delta A_{\mathrm{c}}}\right]=\frac{\mathrm{d} \Phi}{\mathrm{d} A_{\mathrm{c}}}
$$

The subscript $\mathrm{c}$ designates a sensor collector or aperture. The irradiance is a measure of the incident flux per unit area at a point. The total flux is given by

$$
\Phi=\int E \mathrm{~d} A_{\mathrm{c}}
$$

\subsubsection{FIELD ENTITIES}

The terms of radiant exitance, radiant intensity, and radiance are usually thought of as having reference to a source; irradiance on the other hand is considered as having reference to a receiver. However, these concepts can be applied within a radiation field away from a source or receiver. For example, if a barrier containing an aperture is placed in a radiation field, it has the properties of a source for the flux leaving the aperture and a receiver for flux incident upon it. Thus, there is no fundamental reason for distinguishing between the incoming or the outgoing flux. On the contrary, there exists great utility in considering all these as field entities. It is possible, for example, to calculate the flux at any stop, aperture, or detector within a system.

\subsection{SPECTRAL FLUX}

The entities of radiance, irradiance, radiant intensity, and radiant exitance are differential with respect to wavelength (or optical frequency). For example, the average spectral flux is the ratio 
of the total flux integrated over all wavelengths to the total bandwidth. The limiting value of the average spectral flux over a small portion of the spectrum as the bandwidth is reduced to a wavelength (or a wave number) is the spectral flux $\Phi_{\lambda}$ at that wavelength and is given by

$$
\Phi_{\lambda}=\lim _{\Delta \lambda \rightarrow 0}\left[\frac{\Delta \Phi}{\Delta \lambda}\right]=\frac{\mathrm{d} \Phi}{\mathrm{d} \lambda}
$$

which is designated as the spectral density function or concentration. The total flux is given by

$$
\Phi=\int \Phi_{\lambda} \mathrm{d} \lambda
$$

Similar definitions could be written for spectral radiance, spectral radiant exitance, spectral radiant intensity, and spectral irradiance. The entities spectral radiant exitance and spectral radiant intensity are written in abbreviated form as spectral exitance and spectral intensity respectively. In addition, it is recognized that these entities can also be written as a function of wave number.

Table 2 provides a tabulation of the various source spectral entities considering permutations of wavelength or wave number and energy or quanta rate.

\subsection{THE GEOMETRY OF RADIATION TRANSFER}

The calibration of a radiometric sensor consists of a series of experiments in which the sensor response to a standard source is obtained. The radiant flux is transferred from the source to the receiver according to the laws of the geometry of radiation transfer. This geometry is utilized to calculate the flux incident upon the entrance aperture of a sensor during calibration using the geometrical entities defined below and the source characterizations given above. Table 3 summarizes the terms and units pertinent to this section.

\subsubsection{PROJECTED AREA}

The area of a rectilinear projection of a surface (not necessarily a plane surface) onto a plane 
Table 2

SOURCE SPECTRAL TERMS, SYMBOLS, AND UNITS

Energy/Wavelength

Spectral radiance

$L_{\lambda}$

$\left[\mathrm{W} /\left(\mathrm{m}^{3} \mathrm{sr}\right)\right]$ or $\left[\left(\mathrm{W} /\left(\mathrm{m}^{2} \mathrm{sr}\right)\right) / \mu \mathrm{m}\right]$

Spectral exitance

$M_{\lambda}$

$\left[\mathrm{W} / \mathrm{m}^{3}\right]$ or $\left[\left(\mathrm{W} / \mathrm{m}^{2}\right) / \mu \mathrm{m}\right]$

Spectral intensity

$I_{\lambda}$

$[\mathrm{W} /(\mathrm{m} \mathrm{sr})]$ or $[(\mathrm{W} / \mathrm{sr}) / \mu \mathrm{m}]$

Spectral irradiance

$E_{\lambda}$

$\left[\mathrm{W} / \mathrm{m}^{3}\right]$ or $\left[\left(\mathrm{W} / \mathrm{m}^{2}\right) / \mu \mathrm{m}\right]$

Energy/Wave number

Spectral radiance

$L_{\sigma}$

$[\mathrm{W} /(\mathrm{m} \mathrm{sr})]$

Spectral exitance

$M_{\sigma}$

$[\mathrm{W} / \mathrm{m}]$

Spectral intensity

$I_{\sigma}$

[W m/sr]

Spectral irradiance

$E_{\sigma}$

$[\mathrm{W} / \mathrm{m}]$

Quanta/Wavelength

Spectral photon radiance

$L_{\lambda}$

$\left[\mathrm{s}^{-1} /\left(\mathrm{m}^{3} \mathrm{sr}\right)\right]$ or $\left[\left(\mathrm{s}^{-1} /\left(\mathrm{m}^{2} \mathrm{sr}\right)\right) / \mu \mathrm{m}\right]$

Spectral photon exitance

$M_{\lambda}$

$\left[\mathrm{s}^{-1} / \mathrm{m}^{3}\right]$ or $\left[\left(\mathrm{s}^{-1} / \mathrm{m}^{2}\right) / \mu \mathrm{m}\right]$

Spectral photon intensity

Spectral photon irradiance

$I_{\lambda}$

$\left[\mathrm{s}^{-1} /(\mathrm{m} \mathrm{sr})\right]$ or $\left[\left(\mathrm{s}^{-1} / \mathrm{sr}\right) / \mu \mathrm{m}\right]$

$E_{\lambda}$

$\left[\mathrm{s}^{-1} / \mathrm{m}^{3}\right]$ or $\left[\left(\mathrm{s}^{-1} / \mathrm{m}^{2}\right) / \mu \mathrm{m}\right]$

Quanta/Wave number

Spectral photon radiance

$L_{\sigma}$

$\left[\mathrm{s}^{-1} /(\mathrm{m} \mathrm{sr})\right]$

Spectral photon exitance

Spectral photon intensity

$M_{\sigma}$

$\left[\mathrm{s}^{-1} / \mathrm{m}\right]$

$I_{\sigma}$

$\left[\mathrm{s}^{-1} \mathrm{~m} / \mathrm{sr}\right.$ ]

Spectral photon irradiance

$E_{\sigma}$

$\left[\mathrm{s}^{-1} / \mathrm{m}\right]$ 
perpendicular to the direction of the projection is the projected area as illustrated in Figure 1 and is given by

$$
A_{\mathrm{p}}=\int \cos \theta \mathrm{d} A
$$

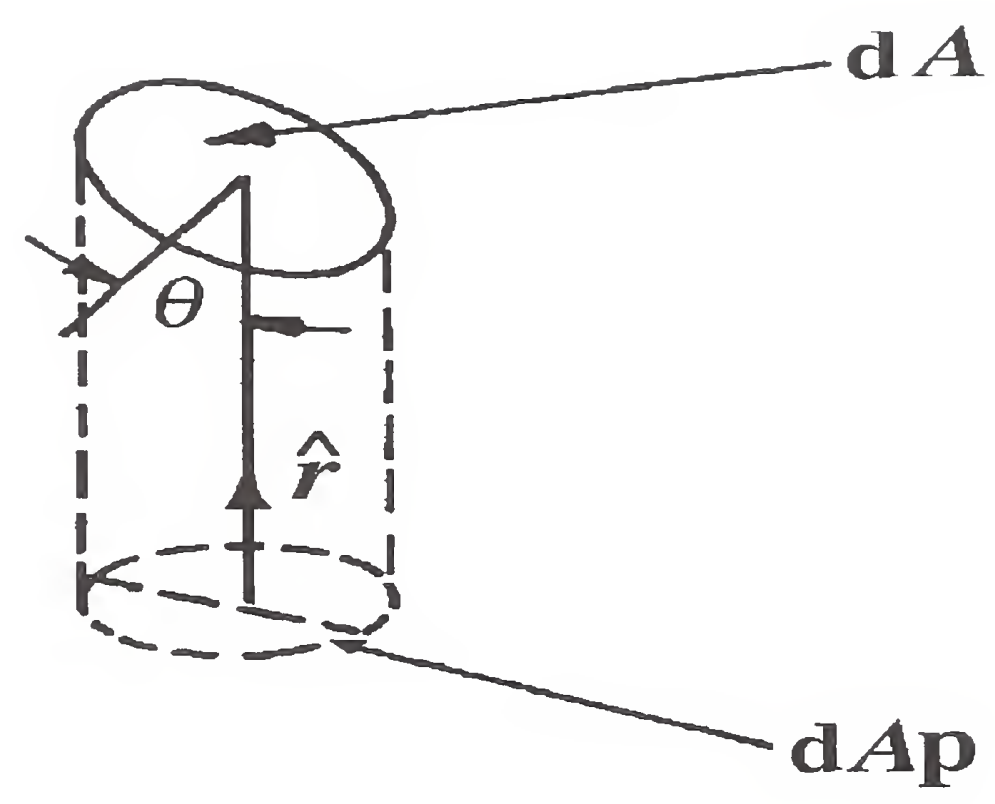

Figure 1. Illustration of the projected area.

\subsubsection{SOLID ANGLE}

The solid angle element $\mathrm{d} \omega$ of a cone formed by straight lines from a single point (the vertex) is numerically equivalent to the area intercepted on the surface of a unit hemisphere centered at the vertex which is illustrated in Figure 2, and $\mathrm{d} \omega=\sin \theta \mathrm{d} \theta \mathrm{d} \phi$. Therefore, the solid angle $\omega$ for a right circular cone with its center on the $Z$-axis will be

$$
\omega=\int_{0}^{2 \pi} \mathrm{d} \phi \int_{0}^{\theta} \sin \theta \mathrm{d} \theta=2 \pi(1-\cos \Theta)
$$


where $\theta$ and $\phi$ are the polar and azimuthal angles respectively and $\Theta$ is the cone half-angle. For a full hemisphere $\Theta$ is equal to $90^{\circ}$, and Eq. 14 yields $\omega=2 \pi \mathrm{sr}$.

\subsubsection{PROJECTED SOLID ANGLE}

The projected solid angle element $\mathrm{d} \Omega$ is the solid angle element $d \omega$ projected on to the plane of observation as shown in Figure 2. It involves another cosine $(\mathrm{d} \Omega=\mathrm{d} \omega \cos \theta)$. For a right cicular cone the projected solid angle $\Omega$ is given by

$$
\Omega=\int \cos \theta \mathrm{d} \omega=\pi \sin ^{2} \Theta
$$

Again, for a full hemisphere $\Theta$ is equal to $90^{\circ}$, and Eq. 15 yields $\omega=\pi$ sr. For small angles i.e. $\Theta$ less than $10^{\circ}$, the value of $\Omega$ will be approximately the same as $\omega$.

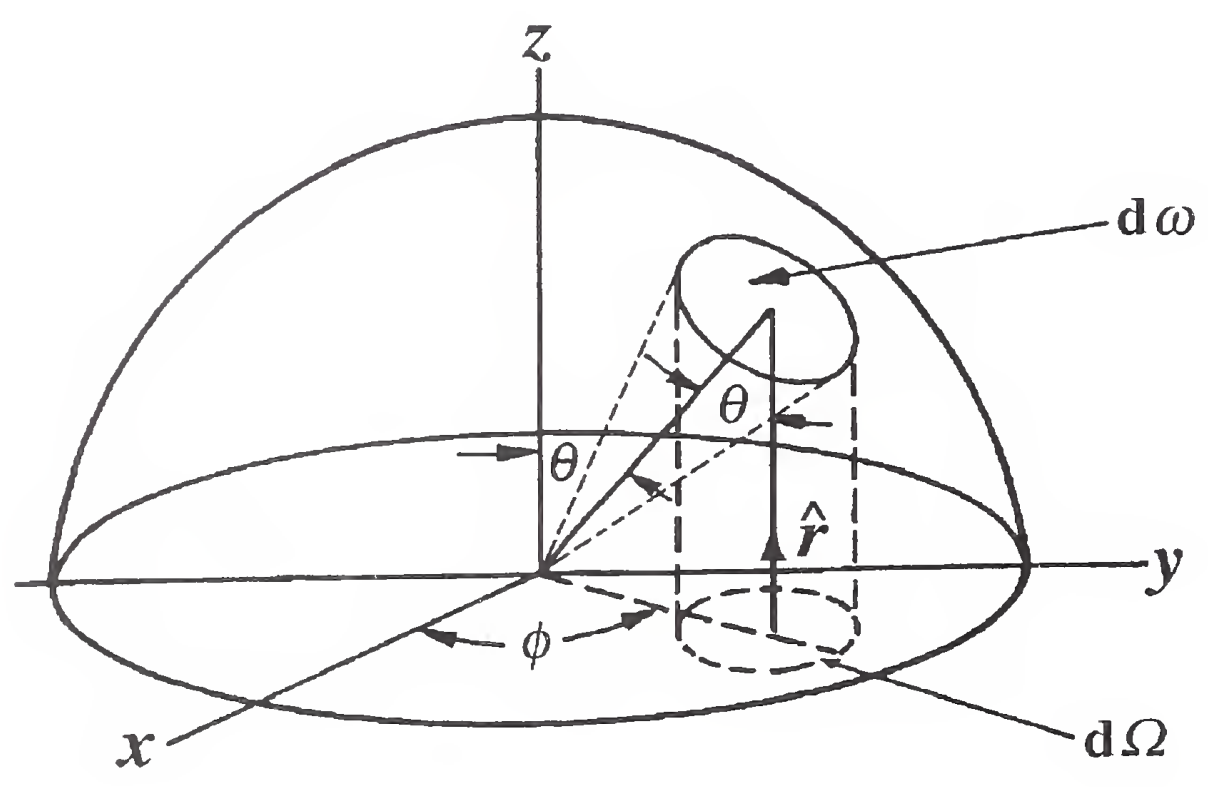

Figure 2. Illustration of solid angle and projected solid angle. 


\subsection{SENSOR PARAMETERS}

The measurement equation includes, in addition to the source and geometry of radiation terms, the sensor parameters as given below. In general, the Greek symbol $\rho$ is used for relative sensor responsivity while the italic $R$ is used for the absolute values. However, the notation of the italic symbol $S_{\mathrm{r}}$ for relative sensor responsivity and the italic $S$ for the absolute value is sometimes used in the literature based on the notation of the International Commission on Illumination (CIE) [3]. There have been considerable discussions between Fred Nicodemus of the National Bureau of Standards (NBS, now NIST) and others in the late 70s [9] on what symbols to be used for these quantities. The use of common symbols for these derived quantities is desirable, but not essential as long as they are properly defined and consistently used in a document. However, the use of common symbols for basic quantities that are connected to SI units is highly recommended as laid out in this document.

\subsubsection{RELATIVE SPATIAL RESPONSIVITY}

If deployed in space, the radiometric sensor aperture is bombarded with unwanted flux which arrives from outside the instrument's field of view, such as the sun, earth, stars, atmosphere etc. The sensor output for a spatially pure measurement is a function of the radiant flux originating from the target (within the sensor field of view) and is completely independent of any flux arriving at the instrument aperture from outside this region. Thus, the characterization of the spatial response, or angular field of view of a sensor, is an essential part of the sensor calibration. In this regard, the sensor relative spatial responsivity $\rho(\theta, \phi)$ is defined as the function that gives the dependence of the spatial responsivity over the sensor's entire hemispherical view relative to the peak responsivity in the direction of its optic axis. Thus, $\rho(\theta, \phi)$ is unitless and is the normalized point-response function which is obtained as the measured off-axis response to a point source. This function can be integrated to provide the solid-angle field-of-view as

$$
\Omega=\int_{(\text {hemisph })} \rho(\theta, \phi) \mathrm{d} \Omega
$$


A detailed discussion on how to determine the sensor field-of-view from the off-axis response to a point source is given in Ch.10 Ref.[15].

\subsubsection{ENCIRCLED (ENSQUARED) ENERGY}

The encircled energy or ensquared energy $\varepsilon_{\mathrm{c}}$ is unitless and is defined as the ratio of the energy incident upon a circular or square detector to the total energy in the image of a point-target on the detector. This image is generally spread out because of imperfect imaging and is called the point spread function. The encircled energy is significant only when the point spread function width becomes a factor in determining the senor response. For example, the simplest case is that the point spread function causes radiation to fall outside the detector active area. An example of a less extreme case is when the point spread function must be averaged over a spatially nonuniform region of the detector. It should be noted that the encircled energy is in general unity for the image of an extended- area source.

\subsubsection{THROUGHPUT AND RELATIVE APERTURE}

Throughput and relative aperture or $f$-number are unitless measures of the "flux gathering power" of an optical system and are defined in reference to Figure 3 where $A_{\mathrm{FS}}$ is the area of the field stop. The sensor throughput is defined as the product of the entrance pupil area $A_{\mathrm{c}}$ and its projected field-of-view $\Omega_{\mathrm{c}},\left[\mathrm{m}^{2} \mathrm{sr}\right]$ and can be written as

$$
A_{\mathrm{c}} \Omega_{\mathrm{c}}=A_{\mathrm{c}} A_{\mathrm{FS}} / f^{2}
$$

which is $A_{\mathrm{c}} \pi \sin ^{2} \Theta$ for a circularly symetrical field-of-view for angles where $\tan \Theta \approx \sin \Theta$.

The relative aperture $F$ or $F / \#$ is given as the ratio of the effective focal length $f$ to the entrance pupil diameter $D$, and is given by the following equation in terms of the index of refraction $n$ and the cone half-angle $\alpha$ for angles where $\tan \alpha \approx \sin \alpha$.

$$
F=f I D=(2 n \sin \alpha)^{-1}
$$




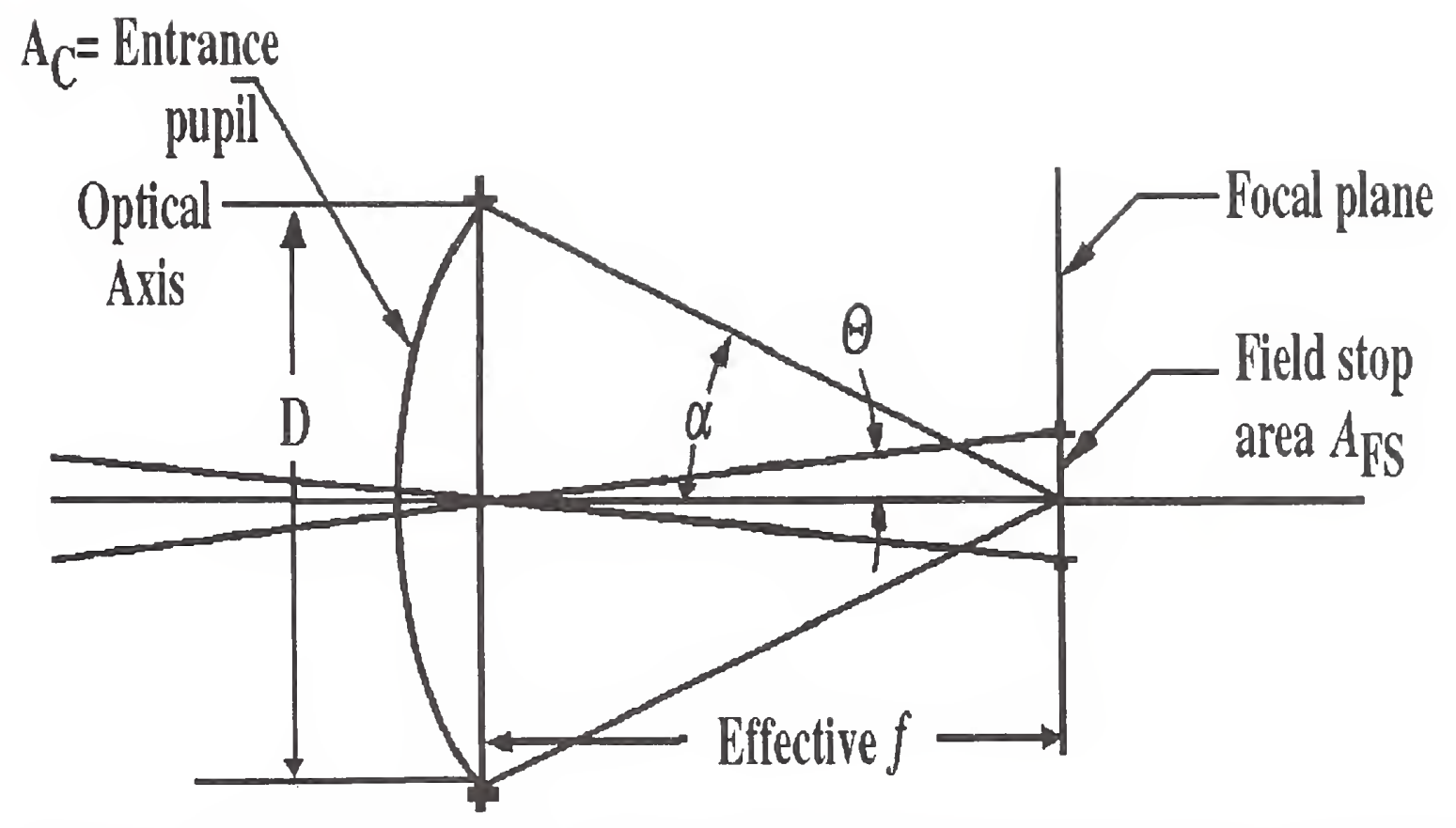

Figure 3. Optical schematic for a simple optical system illustrating the half -angle field-ofview $\Theta$, and the cone half-angle $\alpha$. 
TABLE 3

GONIOMETRIC TERMS AND UNITS

\begin{tabular}{lll}
\hline Polar angle & $\theta$ & [degree] \\
Azimuthal angle & $\phi$ & [degree] \\
Field-of-view half-angle & $\Theta$ & [degree] \\
Relative aperture & $F$ & unitless \\
Cone half-angle & $\alpha$ & [degree] \\
Entrance pupil diameter & $D$ & {$[\mathrm{~m}]$} \\
Projected area & $A_{\mathrm{p}}$ & {$\left[\mathrm{m}^{2}\right]$} \\
Solid-angle & $\omega$ & {$[\mathrm{sr}]$} \\
Projected solid-angle & $\Omega$ & {$[\mathrm{sr}]$} \\
Throughput & $A \Omega$ & {$\left[\mathrm{m}^{2} \mathrm{sr}\right]$} \\
Index of refraction & $n$ & unitless \\
Effective focal length & $f$ & $\mathrm{~m}$ \\
Field stop area & $A_{\mathrm{FS}}$ & $\mathrm{m}^{2}$ \\
\hline
\end{tabular}

Note: The equations containing $\Theta$ are only valid for a solid angle represented as a right circular cone with its central axis coincident with the sensor optical axis, and $\Theta$ represents the half-angle for a circularly symmetric field-of-view. 


\section{Part II: THE RADIOMETRIC SENSOR CALIBRATION}

Radiometric calibration is accomplished in a series of experiments in which the sensor output is observed in response to a number of standard sources. It is necessary to evaluate the sensor performance in the spatial, spectral, and temporal domains as well as to measure the noise and nonlinear characteristics of the system. These experiments are expressed in terms of a measurement equation.

Henry Kostkowski and Fred Nicodemus of the National Bureau of Standards (now NIST) introduced the concept of a "measurement equation."[9][10][11] In order to solve this equation it is necessary to measure pertinent quantities, or obtain all pertinent sensor component specifications from the manufacturer of the sensor. This equation is also called the "system performance equation" in the literature.[10] This equation is especially useful in the efficient design of calibration experiments and evaluating measurement uncertainties.

The measurement equation can also be used to determine calibration coefficients for the reduction of field data to convert sensor response to incident flux. This is obtained by what amounts to an inversion of the dependent and independent variables.

Thus, the calibration equation provides incident radiant flux in terms of the sensor output. In the following, Section 2.1 develops the measurement equations and Section 2.2 provides the corresponding calibration equations for both radiometers and spectrometers.

\subsection{THE MEASUREMENT EQUATION}

The purpose of this section is to begin with a general statement of the measurement equation which is written in terms of sensor and standard source parameters that is valid for radiometers (both spatial and large-field single-detector systems) and spectrometers. Then, solutions of this general measurement equation are illustrated for the specific cases of a spatial radiometer and a spectrometer.

The measurement equation yields sensor output for a specific source configuration. It is a system equation; i.e., it models the sensor system performance in terms of the subsystem and component specifications. Table 4 summarizes the new terms introduced in this section. 
The development given below is based upon the response of a typical detector element of a spatial radiometer and is also valid for a large-field single-detector radiometer. The measurement equation is also valid for a spectrometer. This follows from the concept that the spectrometer provides measurements over a series of sub-bands while the radiometer is considered as the degenerate case of the spectrometer where the number of sub-bands reduces to one.

The equation cannot be written without first making some assumptions: The most general form of the equation assumes the flux is in units of radiance $L$ (positional and directional), is written for wavelength, and the response is given in volts. In general the response could be in digital counts, amperes, film density, pen deflection, etc. The development presented here completely neglects polarization, time, and source coherence and follows the general treatment of the subject given in Ch.5 Ref. [9] where the calibration problems for various applications in radiometry have already been discussed in detail. However, the treatment below illustrates the application of the measurement equation approach for the calibration of a sensor to be deployed in space for observing point sources as well as extended sources.

The general form of the measurement equation illustrates that the response $r$, for a filter radiometer with a chosen filter nominally at $\lambda_{0}$ or for a spectroradiometer at a wavelength setting of $\lambda_{0}$, is obtained by integration over the appropriate variables

$$
r\left(\lambda_{0}\right)=G \iiint L_{\lambda} R_{1}\left(\lambda, \lambda_{0}\right) \cos \theta \mathrm{d} A_{\mathrm{s}} \mathrm{d} \omega_{\mathrm{s}} \mathrm{d} \lambda
$$

where

$\lambda$ is wavelength variable of integration over the spectral bandpass

$G$ is the electronic gain

$L_{\lambda}$ is the source spectral radiance

$R_{\mathrm{I}}\left(\lambda, \lambda_{0}\right)$ is the system absolute (bandpass) spectral responsivity

$A_{\mathrm{S}}$ is the source area

$\omega_{\mathrm{S}}$ is the solid angle subtended by the sensor entrance pupil at the source.

Notice that Eq. (19) is written in terms of source area and the solid angle subtended by the 
detector at the source to make it convenient to use for the case of point sources as well. By reciprocity theorem, Eq. (19) is identical to Eq. (3.11) of Ref. [9]. For the purpose of discussions in this document the subscript $\lambda_{0}$ is mostly redundant and so it will be dropped and then it would be equivalent to Eq. (5.30) in Ch.5 Ref. [9]. However, a comprehensive treatment of Eq. (19) with $\lambda_{0}$ included can be found in chapters 7 and 8 in Ref. [9].

In writing Eq. 19, the absolute bandpass spectral responsivity $R_{\mathrm{I}}(\lambda)$ is assumed to be spatially uniform as a first approximation. It is made up of all wavelength sensitive elements and can be expressed as

$$
R_{\mathrm{I}}(\lambda)=R_{\mathrm{D}}(\lambda) \tau_{\mathrm{F}}(\lambda) \varepsilon_{\mathrm{C}}(\lambda) \gamma(\lambda)=\rho_{\mathrm{l}}(\lambda) \max \left\{R_{1}(\lambda)\right\}
$$

where by definition

$$
\rho_{1}(\lambda)=\frac{R_{1}(\lambda)}{\max \left\{R_{1}(\lambda)\right\}}
$$

is the system relative spectral responsivity, and where

$R_{\mathrm{D}}(\lambda)$ is the detector absolute responsivity

$\tau_{\mathrm{F}}(\lambda)$ is the absolute filter transmittance

$\mathcal{E}_{\mathrm{c}}(\lambda)$ is the encircled or ensquared energy for a point-target. It is a measure of the percent (expressed as a decimal) of the energy in the point-spread function that is incident upon a detector element, and applies to an imaging radiometer. It is unity for an extended source or for a largefield radiometer.

$\gamma(\lambda)$ is the optical efficiency. This term includes reflectance and/or transmittance losses in addition to the filter losses.

The term $\max \left\{R_{\mathrm{l}}(\lambda)\right\}$ is the peak system spectral responsivity over the bandwidth. 
The measurement equation (19) is quite general and is applicable to any radiometry problem involving incoherent radiation. However, there is no unique general solution to this measurement equation. Even if the spectral responsivity $R_{\mathrm{I}}(\lambda)$ is completely characterized as a function of position, direction and wavelength, there are an unlimited number of spectral radiance functions $L_{\lambda}$ that would produce the same observed signal $r$. As observed by Kostkowski and Nicodemus, [Ref.[9] Ch.5], "the practical solution is usually to try to select a measuring instrument and a measurement configuration to satisfy certain conditions, at least with a desired degree of approximation, that will make it possible to modify the measurement equation to include the desired radiometric quantity such as $L_{\lambda}$ and to obtain a unique solution. The kinds of conditions include the constancy or the approximate constancy of a radiation quantity such as $L_{\lambda}$ or of the responsivity relative to one or more radiation parameters so that these functions can be brought outside one or more integrals, the use of an average to replace one or more of the integrals, and the use of the relative spectral distribution, if known such as by using standard sources for calibration, of the otherwise unknown radiometric quantity being measured. The major advantages of using the measurement equation are that it makes clear that such conditions must be sought and provides insight and a systematic approach towards finding them. In fact, without such an approach, it is highly unlikely that one can make state-of-the-art measurements, or even less accurate measurements, with a meaningful estimate of the uncertainty."

For the purpose of this document, as a first step, we assume that the spatial and spectral domains are independent and that the radiance is spatially uniform in Eq. (19) so that the variables can be separated as

$$
r=G \int L_{\lambda} R_{1}(\lambda) \mathrm{d} \lambda \int \cos \theta d \omega_{\mathrm{s}} \int \mathrm{d} A_{\mathrm{s}}
$$

then the indicated integrations can be performed. The integral

$$
\int \mathrm{d} A_{\mathrm{s}} \int \cos \theta \mathrm{d} \omega_{\mathrm{s}}=A_{\mathrm{s}} \Omega_{\mathrm{s}}=A_{\mathrm{c}} \Omega_{\mathrm{c}}
$$

is the source throughput which applies to both the radiometer and the spectrometer, and by the 
invariance theorem [13] is numerically equal to the sensor throughput $A_{\mathrm{c}} \Omega_{\mathrm{c}}$ where $A_{\mathrm{c}}$ is the sensor entrance pupil area and $\Omega_{\mathrm{c}}$ is the projected solid angle subtended by the source at the entrance pupil. $\Omega_{\mathrm{c}}$ is the sensor field-of-view for a uniform extended-area source. For point targets that do not fill the field-of-view it is necessary to make use of the source throughput. The following assumes the appropriate throughput can be represented by $A \Omega$ without subscripts.

The integration of the spectral parameters of Eq. (22) is given using Eq. (21) by

$$
\int L_{\lambda} R_{\mathrm{I}}(\lambda) \mathrm{d} \lambda=\max \left\{R_{\mathrm{I}}(\lambda)\right\} \int L_{\lambda} \rho_{\mathrm{I}}(\lambda) \mathrm{d} \lambda=\max \left\{R_{\mathrm{I}}(\lambda)\right\} L_{\mathrm{N}}
$$

and where for a radiometer

$$
L_{\mathrm{N}}=\int L_{\lambda} \rho_{\mathrm{I}}(\lambda) \mathrm{d} \lambda=\sum_{i=1}^{n} L_{i} \rho_{i} \Delta \lambda_{i}
$$

is the normalized radiance at the radiometer entrance pupil. In other words, it is the effective radiance that is responsible for the sensor output. Note, the normalization applies to the bandpass spectral responsivity [14] and the normalized flux given by Eq. (25) depends upon how this responsivity was normalized. Typically it is peak normalized [14] by the use of Eq. (21) to give the relative spectral responsivity $\rho_{\mathrm{l}}(\lambda)$.

Generally $\rho_{\mathrm{I}}(\lambda)$ is not analytic and can only be represented by a set (array) of numbers obtained in an empirical test. Various methods to measure $\rho_{1}(\lambda)$ independently have been discussed in detail in Ch.7 Ref. [9]. For the calibration of the sensor using a standard source $L_{\lambda}$ is known. In this case the integration can be approximated by numerical methods as illustrated in the right-hand term of Eq. (25) where $\rho_{i}$ is the set of $\mathrm{n}$ values of the response function and $\Delta \lambda_{i}$ is the corresponding wavelength interval. Example 1 shows evaluation of $L_{\mathrm{N}}$ using Eq. (25) for a sensor calibration.

To illustrate the recommended practice, we deduce from Eq.(19) working measurement equations for a broadband radiometer and a high resolution spectral radiometer. 
The measurement equation (19) can be simplified for a radiometer using Eqs. (23) and (24) as

$$
r=G \max \left\{R_{1}(\lambda)\right\} L_{\mathrm{N}} A \Omega
$$

The final and most useful form of the measurement equation is written in terms of the radiance responsivity $R_{\mathrm{L}}$ and the normalized radiance $L_{\mathrm{N}}$ as

$$
r=R_{\mathrm{L}} L_{\mathrm{N}}
$$

where from Eq. (26)

$$
R_{\mathrm{L}}=G \max \left\{R_{1}(\lambda)\right\} A \Omega
$$

Equation (27) is the working measurement equation for a broadband radiometer. It is useful in predicting the radiometer response to an extended or a point source: The radiance responsivity $R_{\mathrm{L}}$ in Eq. (28) is calculated by using a combination of measured and estimated system and component specifications. The gain $G$ is obtained from measurements and system specifications, the throughput $A \Omega$ is calculated using Eq. (23) and $\max \left\{R_{\mathrm{I}}(\lambda)\right\}$ is obtained from Eq. (20) through measurements and system specifications of $R_{\mathrm{D}}(\lambda), \tau_{\mathrm{F}}(\lambda), \varepsilon_{\mathrm{C}}(\lambda)$ and $\gamma(\lambda)$. The normalized radiance $L_{\mathrm{N}}$ is calculated for a particular source using Eq. (25). Analysis of the predicted performance using the measurement equation helps to optimize the design before building the sensor. The calibration of this type of radiometer will be discussed in Section2.2.1.

For a high resolution spectrometer the assumption can usually be made that the spectral radiance $L_{\lambda}$ is constant over the spectral bandpass; then the integration indicated in Eq. (29) yields the spectrometer spectral bandwidth (resolution) $\delta \lambda$ as the normalization factor

$$
L_{\mathrm{N}}=\int L_{\lambda} \rho_{\mathrm{I}}(\lambda) \mathrm{d} \lambda=L_{\lambda}\left\{\int \rho_{1}(\lambda) \mathrm{d} \lambda\right\}=L_{\lambda} \delta \lambda
$$


The spectrometer obtains measurements of the spectral radiance $L_{\lambda}$. Thus, Eq. (26) is written for any sub-band of the spectrometer as

$$
r=G \max \left\{R_{\mathrm{l}}(\lambda)\right\} L_{\lambda} \delta \lambda A \Omega
$$

As with the radiometer, the most useful version of the measurement equation for the spectrometer is given in terms of the spectral radiance responsivity $R_{\mathrm{L}}$ and the spectral radiance $L_{\lambda}$

$$
r(\lambda)=R_{\mathrm{L}}(\lambda) L_{\lambda}
$$

where $R_{\mathrm{L}}$ is given by

$$
R_{\mathrm{L}}(\lambda)=G \max \left\{R_{1}(\lambda)\right\} \delta \lambda A \Omega .
$$

Equations (31) through (32) are valid for any spectrometer sub-band and consequently the spectral radiance responsivity exhibits different values for each sub-band. Equation (31) is the working measurement equation for a high resolution spectral radiometer for each sub-band provided the spectral radiance function is invariant over that bandwidth. The prediction of the performance of a circular variable filter spectral radiometer (CVF) using Eq. (31) is given in example 2 following Section 2.2.3. The calibration of this type of spectroradiometer is discussed in Section 2.2.2. In case the spectral radiance function is not constant over the bandwidth, the measurement problem becomes that of a radiometer and Eq. (27) becomes the working measurement equation at each wavelength setting of the spectroradiometer and normalized radiometric quantity will be the one generally measured.

It is to be noted that working measurement equations, similar to Eqs. (27) and (31) for the respective radiometers can be written for the radiant exitance responsivity, the radiant intensity responsivity, and the irradiance responsivity. For brevity, we will drop the word "working" and simply refer Eqs.(27) and (31) as measurement equations in the rest of the document. 
Sensor response

Detector absolute responsivity

Sensor relative spatial responsivity or field-of-view

Electronic gain

Encircled or ensquared energy

Absolute bandpass spectral responsivity

System relative spectral responsivity

Peak spectral bandpass responsivity

Absolute filter transmittance

Optical efficiency

Source area

Source projected Solid-angle

Sensor throughput

Sensor entrance pupil area

Sensor projected solid-angle

Normalized Radiance $r$

$R_{\mathrm{D}}(\lambda)$

$\rho(\theta, \phi)$

G

$\varepsilon_{\mathrm{c}}(\lambda)$

$R_{\mathrm{I}}(\lambda)$

$\rho_{\mathrm{l}}(\lambda)$

$\max \left\{R_{\mathrm{I}}(\lambda)\right\}$

$\tau_{\mathrm{F}}(\lambda)$

$\gamma(\lambda)$

$A_{\mathrm{s}}$

$\Omega_{\mathrm{s}}$

$\Omega_{c} A_{c}$

$A_{\mathrm{c}}$

$\Omega_{\mathrm{c}}$

$L_{\mathrm{N}}$
[V]

[V/W]

[unitless]

[unitless]

[unitless]

[V/W]

[unitless]

[V/W]

[unitless]

[unitless]

$\left[\mathrm{m}^{2}\right]$

[sr]

[ $\left.\mathrm{m}^{2} \mathrm{sr}\right]$

[ $\left.\mathrm{m}^{2}\right]$

[sr]

$\left[\mathrm{w} /\left(\mathrm{m}^{2} \mathrm{sr}\right)\right]$ 
In developing the measurement equations (27) and (31), the responsivity, of Eq. (20), is termed $R_{\mathrm{I}}(\lambda)$. The subscript "I" comes from the notion that the value of the responsivity is constantly changing as the spectrometer scans; in order that it can be evaluated for a given wavelength it is visualized that we have stopped the spectrometer at that wavelength for an "instant"; thus the term "instantaneous responsivity" often found in literature. The instantaneous responsivity is dominated by the monochromator (filter) and in the ideal, exhibits nonzero values only within the bandpass.

On the other hand, the system responsivity is termed $R_{\mathrm{L}}$. The subscript $L$ comes from the symbol for radiance. The spectral radiance also changes with wavelength as the spectrometer scans. However, in this case the radiance responsivity is dominated by the detector response. Examination of Example 2 shows that for a CVF spectroradiometer, using a Si-As detector, the responsivity is proportional to wavelength squared. The response at $10 \mu \mathrm{m}$ is about 4 times what it is at $5 \mu \mathrm{m}$ and the response at $20 \mu \mathrm{m}$ is about 4 times what it is at $10 \mu \mathrm{m}$. The output voltage provides a distorted representation of the incident spectrum because of this system (detector) weighting.

Equation (27) for the radiometer and Eq. (31) for the spectral radiometer are deduced from Eq. (19) under the assumption that both the radiometric quantity such as $L_{\lambda}$ and the responsivity $R_{\mathrm{I}}(\lambda)$ are uniform and isotropic throughout the beam of radiation incident on the entrance limiting aperture of the radiometer. When the responsivity is uniform and isotropic, but the spectral radiance is not or when the responsivity is not uniform, but the spectral radiance is then Eq. (7.24) or Eq.(7.26) respectively given in Ch.7 Ref. [9] would be valid. In case of spatial nonuniformity of responsivity in the field of view of the sensor, its dependence on $\theta$ and $\phi$ coordinates would be represented by the relative angular responsivity function, $\rho(\theta, \phi)$. In any case, the quantities that are not uniform would be expressed as weighted averages over the incident beam area and the solid angle. If both $L_{\lambda}$ and $R_{\mathrm{I}}(\lambda)$ are not uniform and isotropic throughout the beam of interest then it is best advised that the beam of interest be reduced in size until at least one of the above quantities is sufficiently uniform and isotropic for the accuracy required in solving the measurement Eq.(19). 


\section{Example 1}

Numerical integration of Eq. (25) for the effective radiance in the case of a filter radiometer is given below. The relative response and the spectral radiance are shown in Figure 4 shown below. The bandpass is centered at $2.72 \mu \mathrm{m}$ and the spectral radiance is calculated from Planck's equation for a temperature of $1200 \mathrm{~K}$.

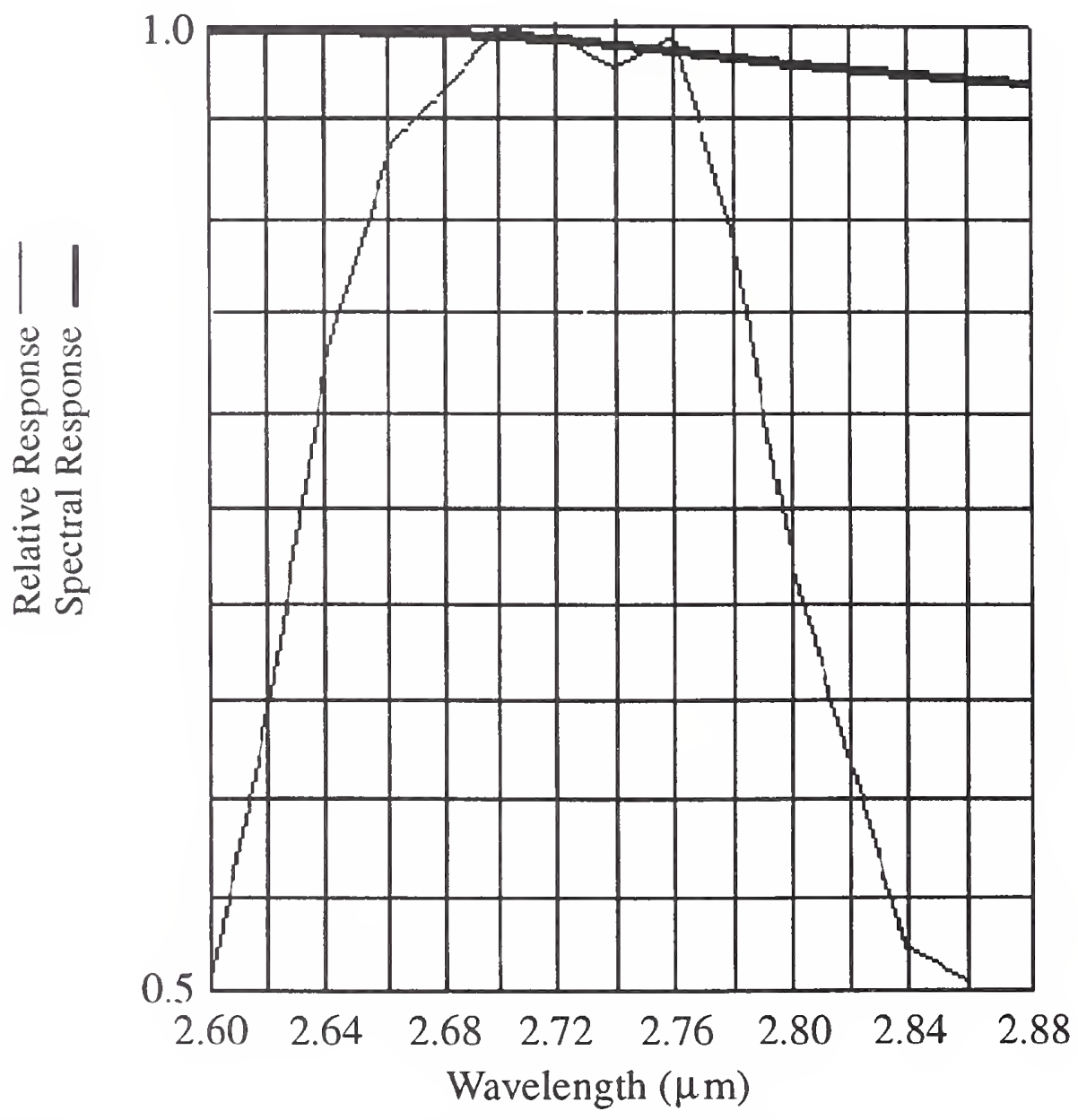

Figure 4. The spectral radiance of a blackbody and the relative response of a filter radiometer. 
The following tabulation gives the index number and corresponding wavelength, relative spectral response, spectral radiance and the product of the spectral response and the spectral radiance. The sum of the products is given at the bottom of the tabulation.

Notice that the wavelength increment is a constant $(0.02 \mu \mathrm{m})$; consequently, Eq. (25) can be expressed as

$$
\begin{array}{lllll}
L_{\mathrm{N}}=\Delta \lambda \Sigma \rho_{i} L_{i}=0.02 \times 8.1519=\left[\mathrm{W} /\left(\mathrm{cm}^{2} \mathrm{sr}\right)\right] \\
\text { Index } \mathrm{i} & \lambda_{i} & \rho_{i} & L_{i} & \rho_{i} L_{i} \\
1 & 2.6 & 0 & 1 & 0.0101 \\
2 & 2.62 & 0.3 & 1 & 0.3 \\
3 & 2.64 & 0.67 & 1 & 0.67 \\
4 & 2.66 & 0.88 & 1 & 0.8774 \\
5 & 2.68 & 0.93 & 0.99 & 0.9244 \\
6 & 2.7 & 1 & 0.99 & 0.99 \\
7 & 2.72 & 0.98 & 0.99 & 0.9663 \\
8 & 2.74 & 0.96 & 0.98 & 0.9427 \\
9 & 2.76 & 0.99 & 0.98 & 0.9682 \\
14 & 2.78 & 0.76 & 0.97 & 0.7402 \\
11 & 2.8 & 0.43 & 0.97 & 0.4167 \\
11 & 2.82 & 0.43 & 0.97 & 0.4167 \\
11 & 2.84 & 0 & 0.96 & 0.0288 \\
& & 0.96 & 0.0955
\end{array}
$$

$$
\Sigma \rho_{\mathrm{i}} L_{\mathrm{i}}=8.1519
$$




\subsection{CALIBRATION EQUATIONS}

In general, the goal of a calibration is to use the working measurement equation to deduce the unknown radiometric quantity by in situ comparison with that of a standard under an identical geometrical setup. In that case, the associated geometrical factors cancel leaving the solution for the unknown radiometric quantity in terms of just the two measured output signals (unknown and the standard) and the known value for the standard. Alternately, the standard could be used to evaluate the responsivity first and then the calibrated responsivity is used in the solution of the measurement equation to measure the unknown quantity from signals measured under the same or known geometrical conditions. In either case the solutions are expressed as equations and are also called the calibration equations. Table 5 summarizes the new terms introduced in Section 2.

In the calibration and uncertainty analysis of complex electro-optical sensors, the goal is first to design calibration experiments using a standard source where necessary, and independently characterize the sensor responsivity in the spectral, spatial, and temporal domains. The working measurement equations such as Eqs.(27) and (31) are generally derived for the major domain that is the spectral part with certain assumptions made regarding the spatial and other domains. Therefore, the quantity that is most important to measure independently is the relative spectral responsivity, $\rho_{1}(\lambda)$ of the sensor system. For spatial and other domains, deviations from the assumptions are assessed and applied as corrections to the measurement equations. Solutions to the modified measurement equations are obtained from the system level results of the calibration experiment and are compared with predictions from component level specifications and measurements. This procedure allows the accurate calibration of the sensor and determination of the overall uncertainty budget. Example 2 at the end of Section 2.2.3 is an illustration of the prediction from component level specifications and measurements for a CVF spectroradiometer. Example 3 given at the end of Section 3 illustrates the system level calibration for the same instrument. Various excellent references are given below that elaborate on the above procedure.

1. The spectral characterization which is the major part of the calibration experiment involves testing for out-of-band leakage, determining the instrument function $\rho_{\mathrm{L}}(\lambda)$, determining the absolute radiance responsivity, $R_{\mathrm{L}}(\lambda)$ and relative spectral responsivity $\rho_{\mathrm{I}}(\lambda)$. It is discussed in detail in Ch.13 Ref. [15]. Also, a comprehensive discussion of various 
methods to determine $\rho_{1}(\lambda)$ can be found in Ch.7 Ref. [9].

2. Regarding other domains of characterization that allow corrections to be made to the measurement equation are noise and possible nonlinearities over the dynamic range of response. A detailed discussion of the evaluation of noise signal can be found in Ch. 8 Ref. [15]. The dark signal results in a constant offset $r_{o}$ which should be subtracted from the raw signal $r_{\mathrm{T}}$ to give the offset corrected signal $r_{\mathrm{oc}}=\left(r_{\mathrm{T}}-r_{\mathrm{o}}\right)$.

All systems exhibit some degree of nonlinearity. The evaluation of the sensor transfer function that corrects for any nonlinearity in the response yields the nonlinear offset correction operator $F_{\mathrm{NL}}$. It is introduced in Section 2.2.1 and is described in Section 3.2.3, but a thorough discussion can be found in Ref. [12] and Ch. 9 Ref. [15].

3. Regarding the spatial characterization:

1. Evaluating the corrections of nonuniformity of pixel to pixel response for the case of an array detector is introduced in Section2.2.1 and is discussed in Section3.2.4. It is called the flat-field correction and is given by the operator matrix denoted by $F_{\mathrm{FF}}$.

2. The spatial field-of-view characterization is very important to assess the sensor performance for the desired linear field-of-view. It is discussed in Section 3.2.5 as angular spatial responsivity characterization. The way to obtain the transfer function for the desired linear field-of-view for the sensor is discussed in Chapters 10 and 11 in Ref. [15]. 3. The Modulation Transfer Function (MTF) is another spatial parameter to be characterized to make necessary corrections and is discussed in Section 3.2.6. A more detailed discussion can be found in Ch.13 Ref. [11].

4. The temporal characterization of the sensor response is discussed in Ch.14 Ref. [15]. Therefore, the calibration experiment which includes evaluation of all the corrections and characterizations listed above yields the sensor response as a function of the radiant, spectral, spatial, and temporal properties of a standard source. The calibration equation is derived from these data in what amounts to an inversion of the measurement equation. This yields the radiant, spatial, spectral, and temporal properties of a target-source as a function of the sensor response. In the next few sections the calibration equations are given for a broad band radiometer and a spectroradiometer. 


\subsubsection{RADIOMETER RADIANCE CALIBRATION EQUATION}

This section uses the imaging radiometer as an example and the measurement Eq. (27) applies for its calibration. The imaging radiometer generates a scene matrix through the use of an area staring array or a linear array and a scanning mirror. The development to follow is valid for each element of a imaging radiometer or for a single element radiometer. For simplicity of expression the following relationships are not expressed in matrix form; however, it must be understood that the indicated relations must be applied to each detector element in the array.

The relative spectral responsivity $\rho_{\mathrm{I}}(\lambda)$ is to be determined first as described in Section 13-3 Ref. [15]. Then the calibration experiment is conducted to measure response $r$ to a standard extended-area source radiance. The normalized radiance $L_{\mathrm{N}}$ from the standard source incident upon the sensor entrance pupil is calculated using Eq. (25). Then the absolute radiance responsivity $R_{\mathrm{L}}$ is obtained from the measurement equation (27). However, in order to use Eq. (27) the response $r_{\mathrm{T}}$ has to be corrected for offset, flat-field and nonlinearity as explained in the previous section. The magnitude of $R_{\mathrm{L}}$ can be determined from a single measurement; but, a redundancy of data is necessary to determine the uncertainty.

The calibration equation is then written in the form of the inversion of the measurement equation, Eq. (27) with all the necessary corrections applied which will be used for measuring the target-source radiance as shown below.

$$
L_{\mathrm{N}}=\frac{r}{R_{\mathrm{L}}}=\frac{1}{R_{\mathrm{L}}} F_{\mathrm{FF}}^{-1} F_{\mathrm{NL}}\left(r_{\mathrm{T}}-r_{0}\right)
$$

where

$r_{\mathrm{T}}$ is the raw response

$r_{\mathrm{O}}$ is the offset correction

$F_{\mathrm{NL}}$ is the nonlinearity correction

$F_{\mathrm{FF}}$ is the flat-field correction

$R_{\mathrm{L}}$ is the absolute radiance responsivity

$L_{\mathrm{N}}$ is the normalized radiance 
TABLE 5

\section{CALIBRATION EQUATION TERMS, SYMBOLS, AND UNITS}

SPECTROMETER: Uncorrected raw response

Offset, linearity, \& flat-field (NUC) corrected response

Absolute spectral radiance responsivity

Peak spectral radiance responsivity

Absolute spectral irradiance responsivity

Peak spectral irradiance responsivity

Instrument function

Spectral resolution

Spectral radiance

Peak spectral radiance

Relative spectral radiance

RADIOMETER : Uncorrected raw response

Offset, linearity, \& flat-field (NUC) corrected response

Offset correction

Offset corrected response

Offset \& nonlinearity corrected response

Absolute radiance responsivity

Absolute irradiance responsivity

Nonlinearity correction

Flat-field correction

Normalized radiance

Normalized target irradiance

Target extraction algorithm

Incremental Bandwidth
$r_{\mathrm{T}}(\lambda)$

[V]

$r(\lambda)$

$R_{\mathrm{L}}(\lambda)$

$\max \left\{R_{\mathrm{L}}(\lambda)\right\}$

$R_{\mathrm{E}}(\lambda)$

$\max \left\{R_{\mathrm{E}}(\lambda)\right\}$

$\rho_{\mathrm{L}}(\lambda)$

$\delta \lambda$

$L_{\lambda}$

$\max \left(L_{\lambda}\right)$

$\varphi(\lambda)$

$r_{\mathrm{T}}$

$r$

$r_{\mathrm{O}}$

$r_{\mathrm{OC}}$

$r_{\mathrm{ON}}$

$R_{\mathrm{L}}$

$R_{\mathrm{E}}$

$F_{\text {NL }}$

$F_{\text {FF }}$

$L_{\mathrm{N}}$

$E_{\mathrm{N}}$

$F_{\text {PT }}$

$\Delta \lambda$
[V]

$\left[\left(\mathrm{V} /\left(\mathrm{m}^{2} \mathrm{sr}\right)\right) / \mu \mathrm{m}\right]$

$\left[\left(\mathrm{V} /\left(\mathrm{m}^{2} \mathrm{sr}\right)\right) / \mu \mathrm{m}\right]$

$\left[\left(\mathrm{V} / \mathrm{m}^{2}\right) / \mu \mathrm{m}\right]$

$\left[\left(\mathrm{V} / \mathrm{m}^{2}\right) / \mu \mathrm{m}\right]$

[unitless]

[m]

$\left[\left(\mathrm{W} /\left(\mathrm{m}^{2} \mathrm{sr}\right)\right) / \mu \mathrm{m}\right]$

$\left[\left(\mathrm{W} /\left(\mathrm{m}^{2} \mathrm{sr}\right)\right) / \mu \mathrm{m}\right]$

[unitless]

[V]

[V]

[V]

[V]

[V]

$\left[\mathrm{V} /\left(\mathrm{m}^{2} \mathrm{sr}\right)\right]$

$\left[\mathrm{V} / \mathrm{m}^{2}\right]$

[unitless]

[unitless]

$\left[\mathrm{W} /\left(\mathrm{m}^{2} \mathrm{sr}\right)\right]$

$\left[\mathrm{W} / \mathrm{m}^{2}\right]$

[unitless]

[m] 
As explained earlier in Section 2.2. the correction operators, correcting the raw response $r_{\mathrm{T}}$ for offset error, nonlinearity, and uniform response over an area array tend to provide an "ideal" radiometer response $r$ expressed in the measurement equation (27). The derivation of each of the corrections $r_{\mathrm{O}}, F_{\mathrm{NL}}, F_{\mathrm{FF}}$ in the above equation is given in Section 3.2.2, Section 3.2.3 and Section 3.2.4 respectively in discussing their uncertainities. Note that $F_{\mathrm{FF}}$ and $F_{\mathrm{NL}}$ represent mathematical operators rather than scalars, namely, the operation of introducing the flat-field (for arrayed systems) and non-linearity corrections. .

In many applications the normalized radiance $L_{\mathrm{N}}$. itself would be the desired radiometric quantity. On the other hand, if radiance $L_{\lambda}$ is the desired radiometric quantity, Eq. (28) has to be deconvolved and the procedures are described in Ch. 8 Ref. [9]. A simplified procedure under special conditions is discussed in Section 3.2.9 in this document for the measurement of the total radiance $L_{\mathrm{T}}$ integrated over the band $\lambda_{1}$ to $\lambda_{2}$ for a broad band radiometer along with associated uncertainty analysis.

\subsubsection{RADIOMETER IRRADIANCE CALIBRATION EQUATION}

This section uses the spatial radiometer as an example. The development to follow is valid for the measurement of an isolated point-target in the scene and derives the scalar target irradiance from the response matrix. This is accomplished with a "target extraction" algorithm which to a first approximation sums the response from those pixels stimulated by the image. The effect of summing the response from several pixels is an improvement in signal-to-noise-ratio. This summing operation is represented by $F_{\mathrm{PT}}$ which includes the algorithm to provide a scalar measure of the incident irradiance from the array image.

The development of the measurement equation and the calibration equation for irradiance follow that given above for radiance; the measurement equation is

$$
r=R_{\mathrm{E}} E_{\mathrm{N}}
$$

where

$r \quad$ is the response 
$R_{\mathrm{E}}$ is the absolute irradiance responsivity

$E_{\mathrm{N}}$ is the normalized irradiance

The calibration equation, Eq. (34) applies to every pixel in the scene array and is written to provide an absolute relationship between the incident flux and the sensor output of the calibration tests. The calibration equation must also make use of the offset, nonlinearity $F_{\mathrm{NL}}$, and array flatfield $F_{\mathrm{FF}}$ corrections for every pixel in the array. In addition, the point extraction $F_{\mathrm{PT}}$ algorithm is used to provide a scaler measure of the incident irradiance from the array image as follows:

$$
E_{\mathrm{N}}=\frac{r}{R_{\mathrm{E}}}=\frac{F_{\mathrm{PT}} F_{\mathrm{FF}}^{-1} F_{\mathrm{NL}}\left(r_{\mathrm{T}}-r_{0}\right)}{R_{\mathrm{E}}}
$$

where $r$ is the corrected or ideal response in the measuement equation. It is noted that the nonlinearity, flat-field and point extractions, terms in Eq. (35) are not factors but operators. These operators have already been discussed in the previous section.

\subsubsection{SPECTROMETER CALIBRATION EQUATION}

This section illustrates the calibration equation for a high resolution spectrometer. The case we are considering applies from the standpoint that the radiometric quantity such as $L_{\lambda}$ does not vary much and can be assumed constant over the bandwidth of the spectrometer at each wavelength setting. The calibration experiment yields the response $r(\lambda)$ in Volts, as a function of wavelength (over the sub-bands), in response to the calibration standard source spectral radiance $L_{\lambda}$ throughout the spectrometer free spectral range (the range in wavelength or wave number for which data are obtained). Again, the raw response $r_{\mathrm{T}}(\lambda)$ has to be corrected for offset and nonlinearity, and also for pixel to pixel variation (flat-field) if an array detector is used as explained in earlier sections resulting in $r(\lambda)$.

The calibration equation is written as the inverse of the the measurement equation. Eq. (31). The response and the spectral radiance responsivity are shown as functions of wavelength (over the sub-bands included in the free spectral range of the spectrometer) as 


$$
L_{\lambda}=r(\lambda) / R_{\mathrm{L}}(\lambda)
$$

A simple scan, yielding the output voltage corresponding to the incident flux for each sub-band in the free-spectral-range provides enough information to calculate $R_{\mathrm{L}}(\lambda)$ for each sub-band. However, a redundancy of data is necessary to test for spectral purity and determine the uncertainties. The procedure for analysing the data to determine $R_{\mathrm{L}}(\lambda)$ is discussed in detail in Section 13-7 Ref.[15]. The uncertainty analysis is given in Sec 3.2.8 in this document.

The nonuniform response over the sub-bands of the spectrometer is considered in the following. The spectral radiance responsivity $R_{\mathrm{L}}(\lambda)$ provides for conversion of output voltage to spectral radiance. The relative variation of $R_{\mathrm{L}}(\lambda)$ over the sub-bands in the free spectral range of the spectrometer can be represented by

$$
R_{\mathrm{L}}(\lambda)=\max \left\{R_{\mathrm{L}}(\lambda)\right\} \rho_{\mathrm{L}}(\lambda)
$$

where $\max \left\{R_{\mathrm{L}}(\lambda)\right\}$ is the maximum value of $R_{\mathrm{L}}(\lambda)$ and $\rho_{\mathrm{L}}(\lambda)$ varies from unity downward.

The resulting calibration equation is written as

$$
L_{\lambda}=\frac{r(\lambda)}{\rho_{\mathrm{L}}(\lambda) \max \left\{R_{\mathrm{L}}(\lambda)\right\}} .
$$

For Eq.(37) the peak responsivity occurs for the sub-band where the relative responsivity is unity. This usually occurs near the peak response of the detector, all other things being equal. The term $\rho_{\mathrm{L}}(\lambda)$ is represented by a set of numbers equal to or less than unity which correct the spectrum for nonuniform $R_{\mathrm{L}}(\lambda)$ over all the spectrometer sub-bands. This function $\rho_{\mathrm{L}}(\lambda)$ is sometimes referred to as the "instrument function." Figures 10 and 13 in the Example 3 at the end of Section3.3.1 illustrate $\rho_{\mathrm{L}}(\lambda)$ for a CVF spectroradiometer.

Equation (38) provides an absolute measure of the spectral radiance; however, as indicated above, spectrometers are not as well suited for absolute measurements as are radiometers. This is 
because the sensor is generally much more complex and the uncertainties are therefore greater.

\section{EXAMPLE 2.}

\section{A CIRCULAR VARIABLE FILTER SPECTROMETER (CVF)}

\section{System specifications:}

Electronic gains - 4 parallel linear output channels for dynamic range :

Absolute gains

$G=2,20,200,2000$ [unitless]

Data Processing:

Coherent rectification

Chopping Factor $=0.33$ [unitless]

Detector: Si As

Current Responsivity $-R_{\mathrm{C}}($ peak $)=1[\mathrm{~A} / \mathrm{W}]$ at $\lambda=23 \mu \mathrm{m}$

Preamplifier:

Transverse impedance $Z=5 \times 10^{9}$ [ohms]

System

$$
\begin{aligned}
& \Omega_{\mathrm{C}}=4.8 \times 10^{-3}[\mathrm{sr}] \\
& A_{\mathrm{C}}=1.98\left[\mathrm{~cm}^{2}\right]
\end{aligned}
$$

CVF

Free spectral Range $=5-22[\mu \mathrm{m}]$

Resolution 3.5 percent

Losses

$\tau_{F}(\lambda) \varepsilon_{C}(\lambda) \gamma(\lambda)=0.059$ [unitless]

(Average including chopping factor) 


\section{Test Conditions:}

Standard blackbody Source Spectral Radiance:

$L_{\lambda}(94.7 \mathrm{~K}, 5 \mu \mathrm{m})=2.42 \times 10^{-13}\left[\left(\mathrm{~W} /\left(\mathrm{cm}^{2} \mathrm{sr}\right)\right) / \mu \mathrm{m}\right]$

$L_{\lambda}(94.7 \mathrm{~K}, 10 \mu \mathrm{m})=3.00 \times 10^{-8}\left[\left(\mathrm{~W} /\left(\mathrm{cm}^{2} \mathrm{sr}\right)\right) / \mu \mathrm{m}\right]$

$L_{\lambda}(94.7 \mathrm{~K}, 20 \mu \mathrm{m})=1.87 \times 10^{-6}\left[\left(\mathrm{~W} /\left(\mathrm{cm}^{2} \mathrm{sr}\right)\right) / \mu \mathrm{m}\right]$

The following analysis shows how to find the output voltage for each gain channel for each source spectral radiance.

\section{Equations:}

$\max \left\{R_{\mathrm{I}}(\lambda)\right\}=R_{\mathrm{D}}(\lambda) \tau_{F}(\lambda) \mathcal{E}_{\mathrm{C}}(\lambda) \gamma(\lambda)[\mathrm{V} / \mathrm{W}]$

$$
\text { for } \rho_{\mathrm{I}}(\lambda)=1 \text { (peak) }
$$

$r(\lambda)=G \max \left\{R_{\mathrm{I}}(\lambda)\right\} L_{\lambda} \delta \lambda A \Omega[\mathrm{V}]$

\section{Discussion:}

A solution of the measurement equation can be obtained through the use of a combination of measured and estimated system and component specifications as illustrated here:

Extended dynamic range is provided through the use of 4 parallel linear output channels. The dynamic range of the high-gain (G2000) channel is given by the ratio of the full-scale output to the rms noise. For the spectrometer used in this example the G2000 rms noise voltage is $37 \mathrm{mv}$ and full scale output is $10 \mathrm{~V}$ giving a dynamic range of 270 . This is extended by a factor of ten for each of the three remain channels to a total dynamic range of 270,000 .

A light chopper, in connection with coherent rectification, is utilized to avoid problems with dc drift. A loss-factor of 0.33 is introduced by the chopper and the noise-bandwidth is increased by a factor of 2 . However these losses are small compared to the improvement achieve by this means.

The photoconductive silicon-arsenic detector is operated at helium temperatures ( 5 to 10 degrees Kelvin) and is modeled as a high-impedance current source. It exhibits a peak current responsivity of about $1 \mathrm{~A} / \mathrm{W}$ at $23 \mu \mathrm{m}$. The variation of responsivity with wavelength is 
approximated with the expression

$$
R_{\mathrm{D}}(\lambda)=R_{C} \text { (peak) } Z \lambda / 23[\mathrm{~A} / \mathrm{W}]=2.17 \times 10^{8} \lambda[\mathrm{V} / \mathrm{W}]
$$

The low-noise preamplifier operates in the "transverse impedance amplifier" (TIA) mode converting the detector current to a voltage so that the voltage responsivity of the detector-TIA system is given by the product of the current responsivity and the impedance of the amplifier.

The monochromator used in this spectrometer is a "circular variable filter" which consists of an interference filter, sometimes referred to as a "wedge" filter because the thickness of the interference layers varies with angular position so that the filter can be made to scan with wavelength as the filter is rotated physically over a slit-detector. The resolution of the filter, to a first approximation, is a fixed percentage of the wavelength, typically 2 to 5 percent. The resolution for this 3.5 percent filter can be modeled as a function of wavelength as

$$
\delta \lambda=.035 \lambda
$$

The losses consist of the peak transmittance of the filter bandpass as well as estimates for other optical losses in lenses and or mirrors and the chopping factor.

The spectral radiances used in this example are taken from a solution to Planck's Equation. A more accurate modeling of the sensor response can often be obtained through the use of measured detector and filter data each of which can be represented as a set of numbers (a linear array) and the use of matrix multiplication to obtain the desired results. Often this requires conversion and re-sampling of the data in order to take the product of two arrays which do not use the same units (wavelength or wave number) or which do not have the same wavelength interval.

$$
\begin{aligned}
& \max \left\{R_{\mathrm{I}}(\lambda)\right\}=2.17 \times 10^{8} \quad \lambda 0.059=1.28 \times 10^{7} \lambda[\mathrm{V} / \mathrm{W}] \\
& r=G 4.26 \times 10^{3} \lambda^{2} L_{\lambda}[\mathrm{V}]
\end{aligned}
$$


Example solution for $G=2$ and $\lambda=20 \mu \mathrm{m}$

$r=2 \times 4.26 \times 10^{3} \times 20^{2} \times 1.87 \times 10^{-6}=6.38[\mathrm{~V}]$

TABULATION OF SOLUTIONS

Output volts as a function of wavelength and gains

$\begin{array}{cllll}\lambda(\mu \mathrm{m}) & \mathrm{G} 2 & \mathrm{G} 20 & \mathrm{G} 200 & \mathrm{G} 2000 \\ 5 & 5.16 \times 10^{-8} & 5.16 \times 10^{-7} & 5.16 \times 10^{-6} & 5.16 \times 10^{-5} \\ 10 & 2.56 \times 10^{-2} & 2.56 \times 10^{-1} & 2.56 & 25.6 \\ 20 & 6.38 & 63.8 & 638 & 6380\end{array}$

The spectral radiance responsivity $R_{\mathrm{L}}(\lambda)$ is obtained as the ratio of the output, given in the tabulation above, to the incident spectral radiance $L(\lambda)$ as given below.

\section{TABULATION OF SOLUTIONS}

Spectral Radiance Responsivity as a function of

wavelength and gains.

$\begin{array}{cllll}\lambda(\mu \mathrm{m}) & \mathrm{G} 2 & \mathrm{G} 20 & \mathrm{G} 200 & \mathrm{G} 2000 \\ 5 & 2.13 \times 10^{5} & 2.13 \times 10^{6} & 2.13 \times 10^{7} & 2.13 \times 10^{8} \\ 10 & 8.53 \times 10^{5} & 8.53 \times 10^{6} & 8.53 \times 10^{7} & 8.53 \times 10^{8} \\ 20 & 3.41 \times 10^{6} & 3.41 \times 10^{7} & 3.41 \times 10^{8} & 3.41 \times 10^{9}\end{array}$




\section{PART III: UNCERTAINTY ANALYSIS}

This section addresses uncertainty analysis in multivariable radiometric systems. The primary contributors to measurement uncertainty are the effects of noise, nonlinearity, nonuniform detector array response, nonideal spectral and spatial responsivity, and standard calibration source uncertainty. Methods used to provide uncertainty estimates have been controversial and methodology has evolved over the years [16]. The approach suggested here is based upon the NIST guidelines (Appendix 2) which are derived from ISO Guide referenced earlier [5].

The uncertainty analysis is an essential part of calibration. The independent characterization of sensor parameters includes estimates of uncertainty which are propagated to a statement of measurement uncertainty.

Statistical analysis of sensor response data is based upon the following assumptions:

1) the source has a large thermal time constant so that it can be regarded as time invariant;

2) the sensor response is not time-dependent, that is, it has no drift;

3) the noise created by the sensor has a normal distribution and its consecutive values are statistically independent of each other (Gaussian white noise);

4) the resulting statistical uncertainty in the measurements is a Gaussian random variable.

Table 6 summarizes the new terms introduced in this section. Please note that in the case when we are dealing with a single detector, the responsivity function is a scalar, while for arrays it is a matrix. This means that respective changes in the mathematical expressions and the physical meaning for these two cases should be kept in mind.

\subsection{DEFINITIONS}

When $N$ statistically independent samples (measurements) of a random variable $\boldsymbol{x}=\left\{x_{1}, x_{2}, \ldots\right\}$ are available, the mean value and variance are defined as

$$
\bar{x}=\frac{1}{N} \sum_{i=1}^{N} x_{i}
$$

and 


$$
s^{2}\left(x_{i}\right)=\frac{1}{N-1} \sum_{i=1}^{N}\left(x_{i}-\bar{x}\right)^{2}
$$

respectively. The positive square root, $s\left(x_{i}\right)$ is the experimental standard deviation. The standard deviation of the estimate of the mean value $\bar{x}$, is

$$
s(\bar{x})=\left[\frac{s^{2}\left(x_{i}\right)}{N}\right]^{1 / 2}
$$

and is measured in units of $x$. The ISO guide defines $s(\bar{x})$ as the standard uncertainty, $u(\bar{x})$. The relative standard uncertainty is given by the ratio of the standard uncertainty [Eq. (41)] to the mean of $x_{\mathrm{i}}$ :

$$
u(\bar{x})=\frac{s(\bar{x})}{\bar{x}}
$$

where $u(\bar{x})$ approaches infinity as the mean tends to zero; however, it is generally taken that radiometric measurements are not useful when the mean value is less than the standard deviation.

In what follows, the relative uncertainty [Eq. (42)] is always used to determine the quality of a measurement and the term "relative" is dropped hereafter for simplicity of expression.

The ISO Guide defines the combined standard uncertainty for $M$ statistically independent components as

$$
u_{c}(y)=\left[\sum_{j=1}^{M} u_{j}^{2}(x)\right]^{1 / 2}
$$

The uncertainty determined by statistical techniques on the basis of direct measurements is referred to in the ISO Guide as Type A while those which are evaluated by other means (e.g., on the basis of a prior assumption), as Type B. Identification and thorough analysis of the components is given in Appendix 2. Finally, the expanded standard uncertainty is denoted in the ISO Guide as $U$ and is obtained for an approximate level of confidence (the interval that will cover 
the true value of the estimated parameter with a given confidence) using the coverage factor $k$.

Thus $U=k u_{\mathrm{c}}(y)$ and the value of the measured parameter $y=y \pm U$ where $\mathrm{y}$ is the measurement result. For example approximately $95 \%$ of the measurements will fall within $\pm 2 u_{\mathrm{c}}(y)$ of the mean which corresponds to the case $k \approx 2$. A $99 \%$ level of confidence corresponds to $k \approx 3$.

Components of uncertainty are developed in the following sections for radiometric and spectrometric measurements using the ISO terminology where applicable. Extensions of the recommended terminology are required in some cases not covered by the Guide.

\subsection{UNCERTAINTY ANALYSIS FOR SENSOR CALIBRATON}

The measurement equations such as Eq. (27) and Eq. (31) form the basis for the uncertainty analysis in the measurement of the radiometric quantities using the calibration equations. The uncertainties associated with corrections to the raw response $r_{\mathrm{T}}$ of the sensor are discussed in Section 3.2.1 through Section 3.2.6. The standard source uncertainty for the calibration experiment using the measurement equation is discussed in Section 3.2.7. The uncertainties in determining the absolute radiance responsivity $R_{\mathrm{L}}$ and associated instrument function $\rho_{\mathrm{L}}$ are discussed in Section3.2.8. Section 3.2.9 and Section 3.2.10 address uncertainties in special measurements using the measurement equations. Section 3.2.9 shows the discussion of uncertainities in determining the radiance of a source in a broad band wavelength interval using the measuement equations for a broad band radiometer. Section 3.2.10 discusses the uncertainty in determining the ratio of radiances between two bands of a broad band radiometer or a spectroradiometer.

\subsubsection{CALIBRATION SNAPSHOTS}

Responsive parameters are measured experimentally, during calibration, using a technique that provides data uncorrupted by noise. This is accomplished using a snapshot of data (a series of measurements) and is based upon the assumption that for a time-invariant calibration source, the dominant uncertainty mechanism is responsivity uncertainty. 
TABLE 6

\section{UNCERTAINTY ANALYSIS SYMBOLS AND TERMS}

\begin{tabular}{ll}
\hline$x_{i}$ & $i^{\text {th }}$ value of variable $x$ \\
$\bar{x}$ & Mean of $x$ \\
$s^{2}(\bar{x})$ & Variance of $x_{i}$ \\
$s(\bar{x})$ & Standard uncertainty or standard deviation of mean of $\boldsymbol{x}$ \\
$u(\bar{x})$ & Relative standard uncertainty of mean of $\boldsymbol{x}$ \\
$y$ & $g(x)$ \\
$u_{\mathrm{C}}(y)$ & Combined standard uncertainty of estimate of $y$ \\
$U(y)$ & Expanded standard uncertainty of estimate of $y$ \\
$\mathrm{u}_{\mathrm{N}}(\bar{r})$ & Noise standard uncertainty (1/SNR) \\
$u_{\mathrm{NL}}(\bar{R})$ & Standard uncertainty of the nonlinearity correction \\
$u_{\mathrm{FF}}(\bar{R})$ & Standard uncertainty of the flat-field correction \\
$u_{\mathrm{FOV}}(\bar{\rho})$ & Standard uncertainty of the spatial responsivity \\
$u_{\mathrm{MTF}}(\bar{r})$ & Standard uncertainty of MTF correction \\
$u_{\mathrm{S}}(\bar{L})$ & Standard uncertainty of the mean radiance \\
$u_{\mathrm{A}}(\bar{R} \mathrm{~L})$ & Standard uncertainty of the absolute radiance responsivity \\
$u_{\mathrm{A}}\left(\overline{R_{\mathrm{E}}}\right)$ & Standard uncertainty of the absolute irradiance responsivity \\
$u_{\mathrm{IF}}\left(\overline{\rho_{\mathrm{L}}}\right)$ & Standard uncertainty of the instrument function \\
$u_{\mathrm{SR}}\left(\overline{\rho_{\mathrm{I}}}\right)$ & \\
$u_{\mathrm{BB}}(\bar{y})$ & Standard uncertainty of the relative spectral response \\
\hline
\end{tabular}


Equation (41) indicates that essentially "noise-free" determinations of the response can be obtained by making $N$ arbitrarily large in the calibration snapshot. It is assumed that uncertainty analysis given in the sections to follow is based upon noise-free response to invariant sources when possible.

\subsubsection{NOISE}

The subject of this section is the uncertainty introduced by random noise in a sensor response $r$ and its effect upon field measurements using the calibration equations. It is essentially a measure of the repeatability of data. The response to a source is obtained at intervals throughout the sensor dynamic range as part of the calibration. In each snapshot a series of $N$ samples, $r=\left(r_{1}, r_{2}\right.$, $\left.\ldots ., r_{N}\right)$, are obtained. The variance of each snapshot response is given by

$$
s^{2}(r)=\frac{1}{N-1} \sum_{i-1}^{N}\left(r_{i}-\bar{r}\right)^{2}
$$

where the mean is given by

$$
\bar{r}=\frac{1}{N} \sum_{i=1}^{N} r_{i}
$$

An estimate of the 1-sigma (one standard deviation, or root-mean-square deviation) confidence interval for a single measurement is given by the ratio of the standard deviation to the mean response following Eq. (42) as

$$
u_{N}(\bar{r})=\frac{s(\bar{r})}{\bar{r}}=\frac{1}{S N R}
$$

This is a Type A component of uncertainty and is defined as the noise standard uncertainty and is equal to the inverse of the signal-to-noise ratio (SNR).

\subsubsection{NONLINEARITY}


This section provides an analysis of uncertainty introduced by nonlinearity in the absolute responsivity $R_{\mathrm{L}}$ or $R_{\mathrm{E}}$ in the measurement equations. This uncertainty applies to the operation of an individual detector either in an array or in single detector systems. Note that in this section, we are interested in the nonlinearity of $R_{\mathrm{L}}$ or $R_{\mathrm{E}}$ and not on absolute values. The uncertainty in their absolute values is discussed in Section 3.2.8.

Nonlinear response in sensor systems is common and has a major impact upon measurement uncertainty. Careful characterization of the sensor response to a spatially, spectrally, and temporally invariant source over the entire dynamic range provides the response $r_{T}$ as a function of the flux $\Phi$. A thorough analysis of the nonlinearity response can be found in Ch. 9 Ref. [15] and Ref. [12].

Analysis here is based upon the data-set described in Section 3.2.1, above. The mean value of each snapshot provides a measure of the offset $r_{\mathrm{O}}$ (dark noise response) and the linearity (throughout the dynamic range). The offset- corrected response $r_{\mathrm{OC}}$ is given as a function of flux $\Phi$,

$$
r_{\mathrm{oc}}=g(\Phi)
$$

The standard source flux is varied for each snapshot but the source is maintained invariant with respect to the spatial and spectral domains in order to isolate nonlinear effects from other uncertainties.

The dependent and independent variables are inverted to provide flux as a function of response:

$$
\Phi=g^{-1}\left(r_{\text {oc }}\right)
$$

which is similar to Eq. (11.8) Ref. 12 and the functional relationship is obtained by best-fit analysis assuming a form for $g^{-1}$.

For example, a second-degree equation has been used for Eq.(48) which is similar to Eq. (9.10) Ref. [15] where the flux is given as

$$
\Phi=a_{1} r_{\mathrm{oc}}+a_{2} r_{\mathrm{oc}}{ }^{2}
$$


for offset-corrected response. The flux for a linear system is given by

$$
\Phi=a_{1} r
$$

Eliminating $\Phi$ between Eqs.(49) and (50) yields the nonlinear offset correction operator $F_{\mathrm{NL}}$ [see Eq. (33)] as

$$
r=F_{\mathrm{NL}}\left[r_{\mathrm{oc}}\right]=r_{\mathrm{oc}}+\frac{a_{2}}{a_{1}} r_{\mathrm{oc}}{ }^{2}
$$

which indicates that the nonlinearity is described by the ratio of $a_{2}$ to $a_{1}$. Mathematical modeling to arrive at $F_{\mathrm{NL}}$ i.e $a_{1}$ and $a_{2}$ is given in Section 9.3 Ref.[15].

Figure 5 illustrates the ideal linear system transfer function as a solid line with the offset and linearity corrected response data superimposed. These data used the form of Eq. (51) but were partitioned to reduce the uncertainty.

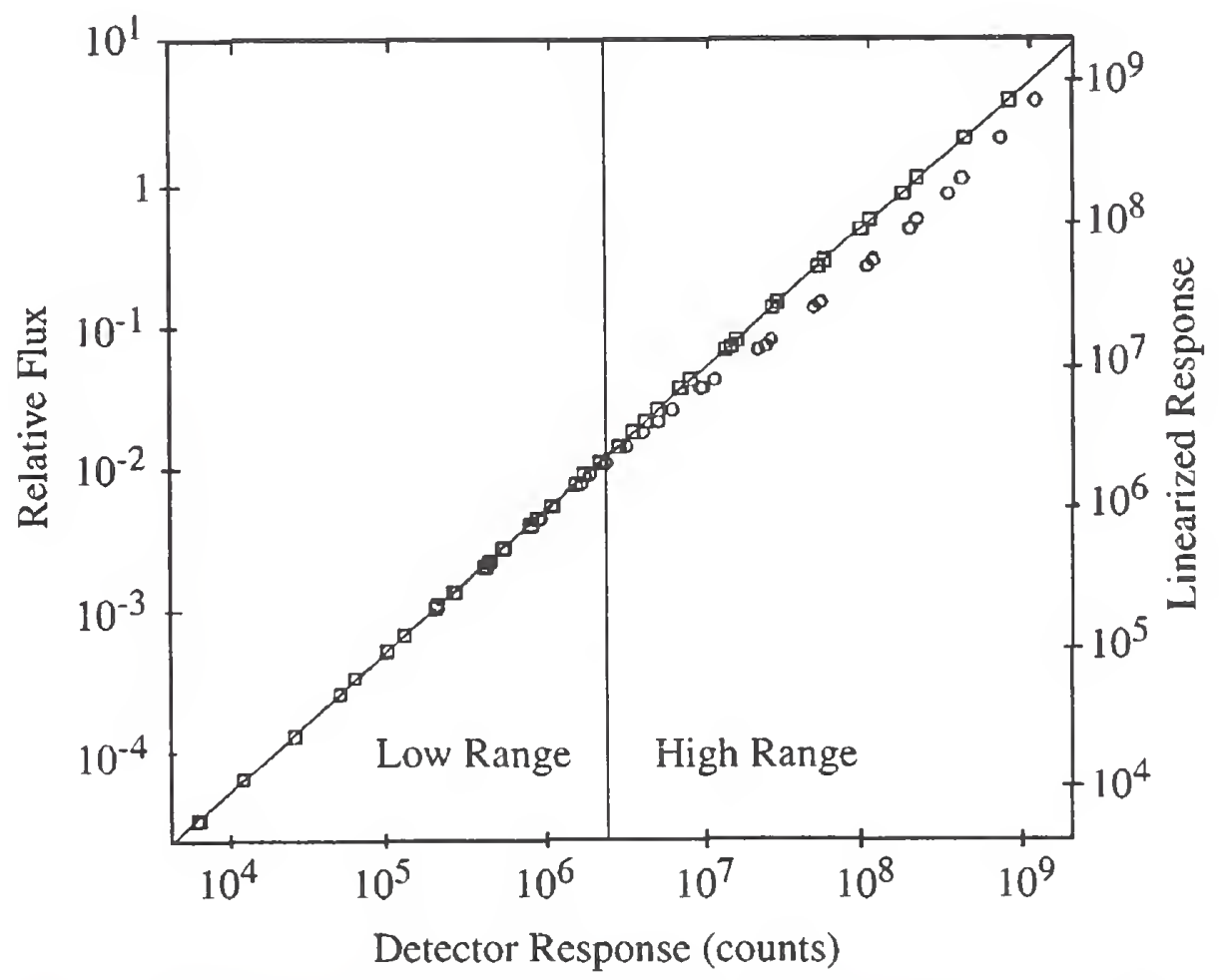

Figure 5. Illustration of data linearization. The original data - circle, linearized data-square, solid curve is the ideal linear response. 
The sensor offset and nonlinearity corrected response $r$ yields the nonlinearity corrected responsivity $R$ (radiance or irradiance), for the calibration test data. It is given by

$$
R=\frac{r_{\text {oc }}}{\Phi}
$$

which should be constant for a well behaved sensor. However, evaluation of the data typically indicate variations in responsivity. The effect of noise has been reduced since each datum represents the mean of a snapshot. The principle cause of the variations is most likely in not knowing the correct form for Eq. (48). In any case, the uncertainty introduced by these observed variations in the offset and nonlinearity corrected responsivity must be characterized. Equation (52) is evaluated at various flux levels of the standard radiometric source output at various times to test for reproducibility. Regression analysis is performed to find the best-fit equation such as Eq. (51) and the standard deviation is obtained .

The standard uncertainty of nonlinearity correction, a Type A uncertainty, is herein defined as the ratio of the standard deviation to the mean corrected responsivity

$$
u_{\mathrm{NL}}(\bar{R})=\frac{s_{\mathrm{NL}}(\bar{R})}{\bar{R}}
$$

where $\bar{R}$ is the mean corrected responsivity and $s_{\mathrm{NL}}(\bar{R})$ is the standard uncertainty of the mean of $R$.

\subsubsection{NONUNIFORM AREA RESPONSIVITY}

This section deals with uncertainty in determining any spatial nonuniformity of responsivity, the important quantity in the measurement equations. Variations in the responsivity of individual detector elements over an area array must be corrected in scene data. This is accomplished in a calibration experiment where the array is flooded with a spatially uniform source at one irradiance level for a single point calibration. 
$N \times M$ elements of the matrix, $F_{\mathrm{FF}}(i, j)$ of corrections are derived from the data:

$$
F_{\mathrm{FF}}(i, j)=\frac{r_{\mathrm{ON}}(i, j)}{\bar{r}_{\mathrm{ON}}}
$$

where $r_{\mathrm{ON}}(i, j)$ is the offset and nonlinearity corrected response matrix and $\bar{r}_{\mathrm{ON}}$ is the mean (offset and nonlinearity corrected) response given by

$$
\bar{r}_{\mathrm{ON}}=\frac{1}{N M} \sum_{i=1}^{N} \sum_{j=1}^{M} r_{\mathrm{ON}}(i, j)
$$

The corrected response, $r(i, j)$ for field data, $\left[r_{\mathrm{ON}}(i, j)\right]_{\text {field }}$ is given by

$$
r(i, j)=\frac{\left[r_{\mathrm{ON}}(i, j)\right]_{\text {field }}}{F_{\mathrm{FF}}(i, j)}
$$

The quantity $r(i, j)$, corrected for offset, nonlinearity, and flat-field, is called the Nonuniformity Corrected Response (NUC)[17].

An example is as follows: Given the response of a particular detector element is 68 counts and the mean response is 81 counts for a single point calibration; according to Eq.(54), the correction factor is 0.84 for that detector element. By Eq.(56) the corrected response is, as expected, equal to the mean response, 81 counts.

The corrected responsivity, according to Eq. (52) is therefore given by

$$
R(i, j)=\frac{r(i, j)}{\Phi}
$$

which, for an ideal array, is uniform over all detectors at all irradiance levels. However, in reality there will be variations in responsivity because of residual non linearities in the detector elements.

Therefore, the standard uncertainty of the flat-field correction $u_{\mathrm{FF}}(R)$, a Type A uncertainty is given by the ratio of the standard uncertainty of the corrected responsivity to the mean 
responsivity over the irradiance levels of interest. This is expressed as

$$
u_{\mathrm{FF}}(\bar{R})=\frac{s(\bar{R})}{\bar{R}} .
$$

\subsubsection{ANGULAR SPATIAL RESPONSIVITY}

In this section, the uncertainty of determining any angular dependence of responsivty to correct for it in the measurement equations is analyzed. A single detector system with a wide angle nonuniform field-of-view is discussed. The sensor responsive parameters are independently determined by experimentally evaluating the response to a standard source whose properties are independent of direction and time. For example, the relative spatial response $\rho(\theta, \phi)$ of a practical IR sensor exhibits nonuniform response to a point source over the field-of-view as illustrated in Figure 6. It is assumed that the sensor pointing accuracy is adequate to guarantee that the target falls somewhere within the "inner-core" of the field-of-view but that its exact location within the inner-core cannot be known. It may also be assumed that the probability is equally likely that it will fall anywhere within the inner-core but zero that it will fall outside the inner-core.

The problem of uncertainty analysis in this case is to characterize the uncertainty associated with spatial variations in responsivity within the inner-core. Calibration of the spatial response function $\rho(i, j)$ consists of mapping the relative response to a point target. The absolute calibration of the sensor is "normalized" to some arbitrary point in the inner-core of the field-of-view. The relative error $\varepsilon(i, j)$ associated with a target imaged on the $(i, j)$ th grid position is given by the ratio of the relative spatial response $\rho(i, j)$ to the normalizing relative response $\rho_{N}$ minus one :

$$
\varepsilon(i, j)=\frac{\rho(i, j)-\rho_{\mathrm{N}}}{\rho_{\mathrm{N}}}
$$

For example, assuming peak normalization, i.e., $\rho_{\mathrm{N}}=1$ and $\rho(i, j)=0.85$; the error as given by Eq. (59) is -0.15 . This means, that neglecting all other errors, the sensor underestimates the measured flux by $15 \%$. 
Peak normalization introduces systematic error; that is, the sensor always underestimates the flux as discussed in Ch.5, Eq. (5-45), Ref [9]. Consequently, it is preferred to normalize to the inner-core average $\bar{\rho}$ so that in the long-run the error will tend to average out.

The relative error expressed by Eq. (59) can be rewritten in terms of the inner core average $\bar{\rho}$ as

$$
\varepsilon(i, j)=\frac{\rho(i, j)}{\bar{\rho}}-1=\frac{1}{\bar{\rho}}[\rho(i, j)-\bar{\rho}]
$$

and from Eqs. (40) and (41) the standard uncertainty is given by

$$
u_{\mathrm{FOV}}(\bar{\rho})=\frac{s(\bar{\rho})}{\bar{\rho}}
$$

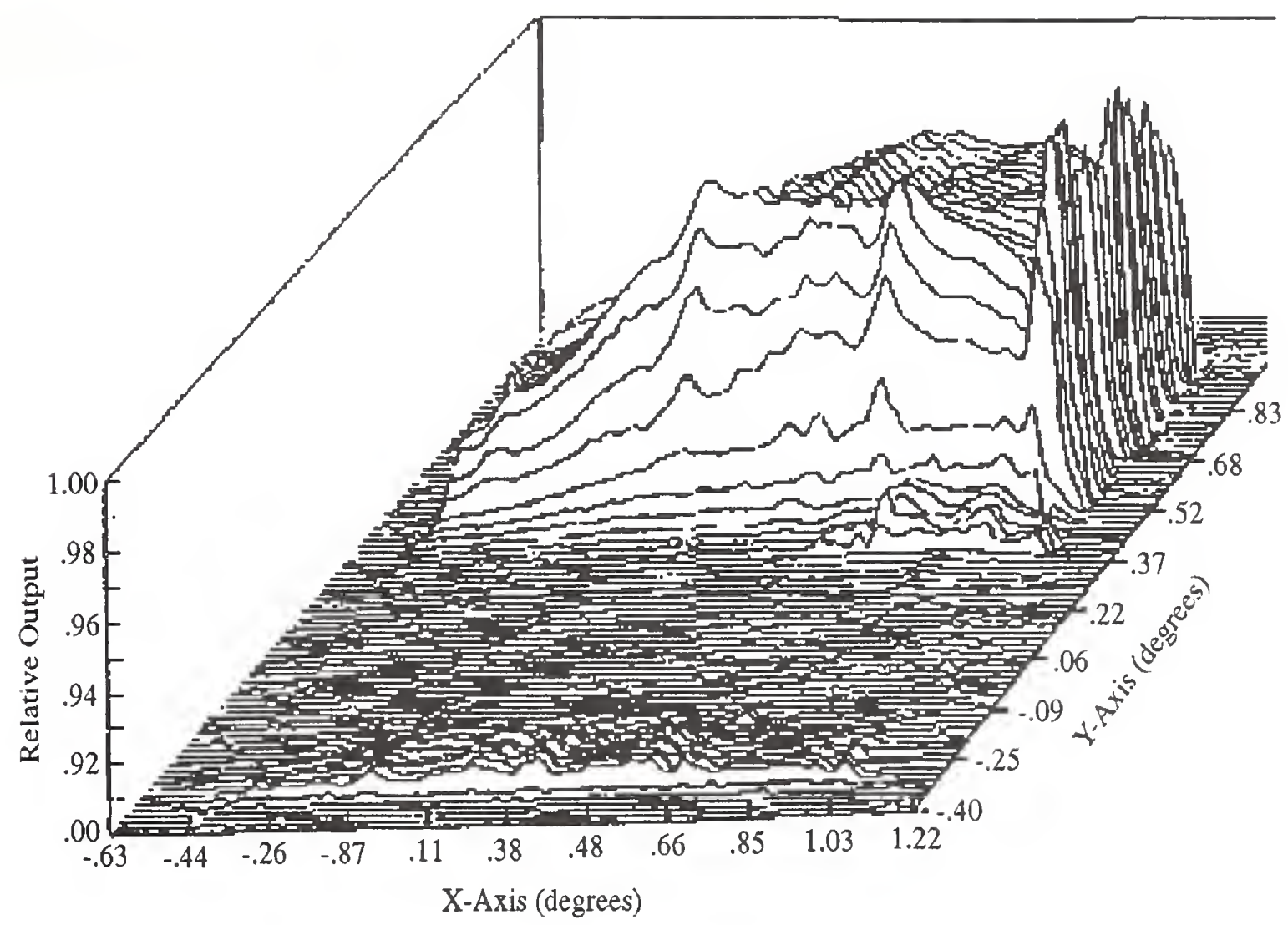

Figure 6. Isometric 3 dimensional view of the spatial response of an infrared detector. 
This is recognized as the ratio of the standard uncertainty of the mean relative response to the mean response. The quantity $u_{\text {Fov }}(\bar{\rho})$ given by Eq.(61), is Type A uncertainty and is herein defined as the standard uncertainty of the spatial responsivity.

\subsubsection{MTF CORRECTION UNCERTAINTY}

This section deals with the response $r$ in the measurement equation of the imaging sensor system. The modulation transfer function (MTF) of a spatial radiometer provides a measurement of the resolution in terms of a frequency response function in the spatial domain. In other words its value represents how well the image replicates the object. For an ideal system MTF is by definition unity which is assumed to be the case for the sensor in writing the measurement equation. Deviations from the ideal system are to be measured and corrected in the system response in applying the measurement equation. Calculation and measurement of MTF also helps in optimizing the design of the sensor to meet the specifications. It is discussed in Ch.13 Ref.[10].

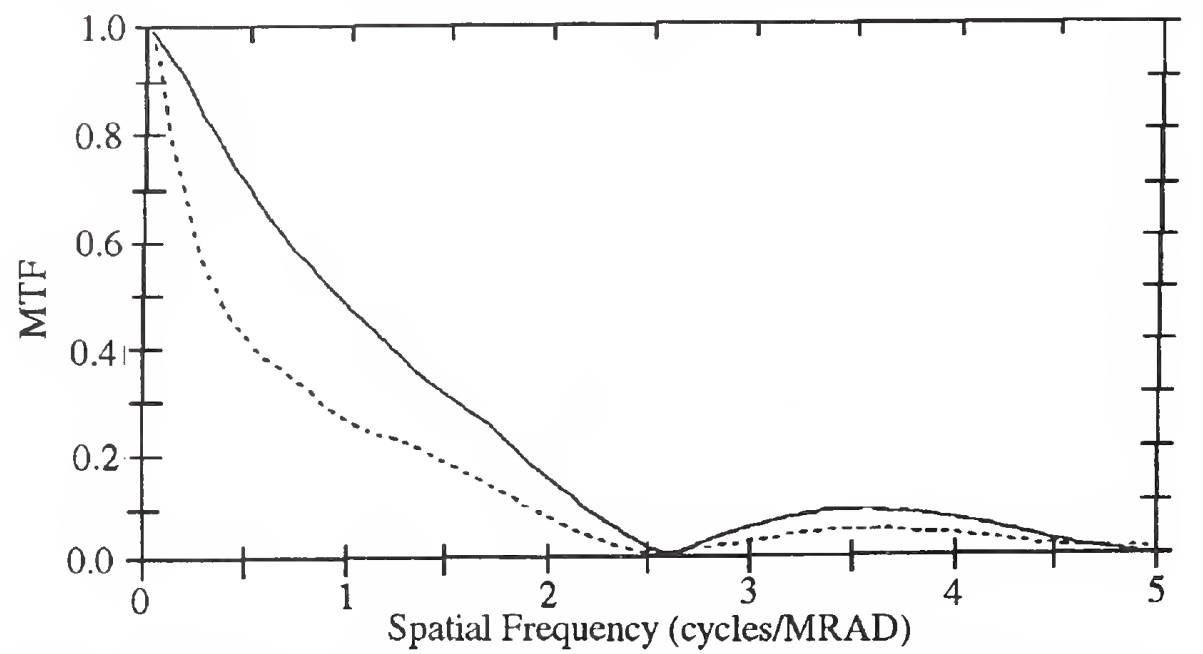

Figure 7. Point (----) and line spread ( ) function MTF for a sensor.

Uncertainties in the MTF result from optical scattering that varies with the scene. The MTF can be calculated from the point response data for point- and line-spread functions that provide an estimate of MTF uncertainty for nonuniform extended-area sources.

Figure 7 is an example of the MTF of a spatial sensor for the point- and line-spread functions. It is observed that the line-spread function MTF is degraded by optical scattering. 
It is likely that the best estimate of the MTF lies between the two bounds for practical source spatial distributions and is zero outside that range and that any value of MTF (for a given spatial frequency) within that range is equally probable. In this case the best estimate is the midpoint:

$$
\overline{M T F}=\frac{1}{2}[M T F(\text { line })+M T F(\text { point })]
$$

and if the difference [MTF(point) - MTF(line)] be denoted by $2 a$, then the standard uncertainty of the MTF correction, which is a Type B component, is given by [Appendix 2, 4.6]

$$
u_{\mathrm{MTF}}(\bar{r})=u(\overline{M T F})=\left[\frac{a^{2}}{3}\right]^{1 / 2}
$$

\subsubsection{CALIBRATION STANDARD SOURCE UNCERTAINTY}

Generally, blackbody simulators serve as calibration standards for sensor systems to measure and evaluate unknowns in the measurement equation. Commercial sources are available which exhibit large thermal time constants and may be considered noise-free. Cavity blackbody simulators exhibit emissivity values close to unity; consequently, the source uncertainty is related primarily to temperature uncertainty. Blackbody flux uncertainty can be related to temperature uncertainty using Planck's equation. NIST performs calibrations of blackbody sources and provides uncertainty estimates for each temperature setting.

The effective flux for a radiometric calibration can be obtained by Wien distribution law, an approximation to the Planck's equation. Considering the Planck's function to be essentially not varying over the spectrometer bandwidth $\delta \lambda$ :

$$
L=\int L_{\lambda} \mathrm{d} \lambda=c_{1} \lambda^{-5}\left[\exp \left(c_{2} / \lambda T\right)\right]^{-1} \delta \lambda
$$

where the radiation constants, $\mathrm{c}_{1}=1.1910^{-16}\left[\mathrm{~W} . \mathrm{m} . \mathrm{sr}^{-1}\right]$ and $\mathrm{c}_{2}=1.4410^{-2}[\mathrm{~m} . \mathrm{K}]$. This 
approximation to the Planck's equation is good to better than $1 \%$ for all $(\lambda . T)<3.110^{-3}[\mathrm{~m} . \mathrm{K}]$. The rate of change of radiance with temperature is obtained by taking the derivative of Eq. (62) with respect to temperature. Therefore,

$$
\Delta L=\frac{c_{1} c_{2} \Delta T}{T^{2} \lambda^{6}}\left[\exp \left(c_{2} / \lambda T\right)\right]^{-1} \delta \lambda
$$

where $\exp \left(\mathrm{c}_{2} / \lambda T\right)>>1$.

Typically, the temperature uncertainty is specified as $T \pm \Delta T$. The corresponding radiance uncertainty is $L \pm \Delta L$. When there is no specific information about the possible values of $L$ within the range, it may be assumed that it is equally probable for $L$ to lie within the range of $L-\Delta L$ to $L+\Delta L$ and the probability that $L$ lies outside this range is zero. Then, according to the ISO Guide [4], the best estimate of the source radiance is at the midpoint $\bar{L}$ (given by Eq.(62) and the source variance is

$$
s_{S}^{2}(L)=\Delta L^{2} / 3
$$

The standard source uncertainty is therefore

$$
u_{S}(\bar{L})=\frac{s_{S}(\bar{L})}{\bar{L}}=\frac{\Delta L}{\sqrt{3} L}
$$

It corresponds to the standard uncertainty of the mean radiance which is a Type B component of uncertainty.

\subsubsection{ABSOLUTE RESPONSIVITY UNCERTAINTY}

The topic of this section deals with the absolute responsivity (radiance or irradiance) in the measurent equations such as Eq.(27), Eq. (31) and Eq. (34). First we deal with the absolute calibration of the radiometer and then with the spectrometer.

The absolute responsivity is determined in a series of tests in which the NUC response is 
observed as the standard source temperature is varied over the range of values expected in the field measurements. This test is often referred to as the "spectral purity" or "out-of- band leakage" calibration test as discussed in Ch.13-5 Ref. [15]. As the temperature of the source is varied, the flux is shifted relative to possible leak regions. Excessive response is observed when the wavelength of peak flux is shifted into a leak region.

The results of the spectral purity test can be examined for a systematic change in responsivity as a function of the calibration standard source temperature. In the absence of a systematic change, the mean responsivity may be the best estimate of the absolute responsivity. As with the nonlinearity, it is the absolute responsivity that should be invariant.

The uncertainty resulting from the observed variations must be estimated as follows: The absolute radiance responsivity is given by

$$
R_{\mathrm{L}}=r / L_{\mathrm{N}}
$$

where $L_{\mathrm{N}}$ depends upon the source temperature and the relative spectral responsivity $\rho_{1}(\lambda)$. The standard uncertainty of the absolute radiance responsivity $u_{\mathrm{A}}\left(\bar{R}_{\mathrm{L}}\right)$, which is a type A component, is obtained as the ratio of the standard uncertainty of the absolute radiance responsivity to the mean absolute radiance responsivity:

$$
u_{\mathrm{A}}\left(\bar{R}_{\mathrm{L}}\right)=\frac{s\left(\bar{R}_{\mathrm{L}}\right)}{\bar{R}_{\mathrm{L}}}
$$

where $\bar{R}_{\mathrm{L}}$ is the mean radiance responsivity and $s\left(\bar{R}_{\mathrm{L}}\right)$ is the standard deviation of $\bar{R}_{\mathrm{L}}$.

Similar terms can be written for the standard uncertainty of the absolute irradiance responsivity $s\left(\bar{R}_{\mathrm{E}}\right)$.

Spectrometers can also be calibrated for absolute spectral radiance measurements, as given in Examples 2 and 3, although they are generally utilized to measure the relative spectrum. The spectral purity test is also used in the case of the spectrometer, the difference being that the uncertainty must be determined for each sub-band of the spectrometer and is therefore a function of wavelength, 


$$
u_{\mathrm{A}}\left[\bar{R}_{\mathrm{L}}(\lambda)\right]=\frac{s\left[\bar{R}_{\mathrm{L}}(\lambda)\right]}{\bar{R}_{\mathrm{L}}}
$$

The mean responsivity is given by the ratio of the mean response to the incident spectral radiance:

$$
\vec{R}_{\mathrm{L}}(\lambda)=\frac{\bar{r}(\lambda)}{L_{\lambda}}
$$

where $L_{\lambda}$, which is the spectral radiance, depends upon the source temperature and the wavelength. The experimental procedure for measuring the absolute radiance responsivity is discussed in Section 13-6 in Ref. [15]. Figure 9 and Figure 12 in Example 3 given at the end of Section 3.3.1 show the standard uncertainty of the absolute radiance responsivity determined for a CVF spectroradiometer using an extended source and a point source respectively.

The "instrument function" is defined in Section 2.2.3 as the normalized spectral radiance responsivity $\rho_{\mathrm{L}}(\lambda)$, see Eq. [37],

$$
R_{\mathrm{L}}(\lambda)=\max \left\{R_{\mathrm{L}}(\lambda)\right\} \rho_{\mathrm{L}}(\lambda)
$$

which is

$$
\bar{\rho}_{\mathrm{L}}(\lambda)=\frac{\bar{r}(\lambda)}{L_{\lambda} \max \left\{R_{\mathrm{L}}(\lambda)\right\}} .
$$

The scatter in the sensor absolute responsivity, observed in the spectral purtiy test, for offset and nonlinearity corrected response applies equally to the relative spectral radiance responsivity $\rho_{\mathrm{L}}(\lambda)$ which is the instrument function. Therefore the standard uncertainty of the instrument 
function $u_{\mathrm{IF}}\left(\bar{\rho}_{\mathrm{L}}\right)$, which is a Type A component, is obtained using equation similar to Eq. (69).

\subsubsection{NONUNIFORM SPECTRAL RESPONSE}

This section deals with uncertainties that result from nonideal spectral bandpass response characteristics of radiometers as opposed to errors in determining the spectral response function considered in Section3.2.8. These uncertainties exist even when the spectral response function is known to a high degree of confidence. Only specific cases are considered here and a thorough analysis can be found in Ch. 8 Ref. [9].

The data analyst can correct for this type of uncertainty provided sufficient supplemental data are available from measurement or theory. In the absence of such data, the analyst must provide uncertainty estimates. These may be systematic uncertainties. The error introduced by nonideal performance parameters can be catastrophic if no provisions are undertaken to make corrections,

Errors associated with the nonideal spectral response can best be understood in terms of a measurement goal, which is to obtain a measure of total radiance $L_{\mathrm{T}}$ integrated over the spectral range of $\lambda_{1}$ to $\lambda_{2}$. This can be expressed as

$$
L_{\mathrm{T}}=\int_{\lambda_{1}}^{\lambda_{2}} L_{\lambda} \mathrm{d} \lambda
$$

The actual output response from a radiometer is given by the measurement equations, Eqs. (24), (25), and (26). Therefore,

$$
r=G A \Omega \max \left\{R_{1}(\lambda)\right\} \int_{0}^{\infty} \rho_{1}(\lambda) L_{\lambda} \mathrm{d} \lambda
$$

where $\rho_{\mathrm{I}}(\lambda)$ is the sensor relative spectral response function, the integration is carried out over all wavelengths, and $\max \left\{R_{\mathrm{I}}(\lambda)\right\}$ is the systems peak responsivity.

The best that can be done is to obtain the measured radiance $L_{\mathrm{M}}$ implied by Eq. (75): 


$$
L_{\mathrm{M}}=r\left[A \Omega G \max \left\{R_{\mathrm{I}}(\lambda)\right]^{-1}=\int_{0}^{\infty} \rho_{\mathrm{I}}(\lambda) L_{\lambda} \mathrm{d} \lambda\right.
$$

However, based upon Eq. (76), $L_{\mathrm{M}}$ is equal to $L_{\mathrm{T}}$ only if

$$
\int_{\lambda_{1}}^{\lambda_{2}} L_{\lambda} d \lambda=\int_{0}^{\infty} \rho_{1}(\lambda) L_{\lambda} \mathrm{d} \lambda
$$

The ideal sensor is one for which $\rho_{1}(\lambda)$ is unity between $\lambda_{1}$ and $\lambda_{2}$ and is zero elsewhere; then Eq. (77) is identically true.

Unfortunately, the spectral response function is never ideal in practical sensors. A spectral bandpass filter is illustrated in Figure 8. The nonuniform transmittance and finite slope are typical of any physically realizable filters.

For a practical sensor, Eq.(77) is true for the limiting case where the radiance is uniform (i.e., $L_{\lambda}=$ const) over the entire responsive region, that is, for nonzero values of $\rho_{1}(\lambda)$. Then Eq. (77) becomes

$$
\int_{\lambda_{1}}^{\lambda_{2}} \mathrm{~d} \lambda=\int_{0}^{\infty} \rho_{1}(\lambda) \mathrm{d} \lambda
$$

and gives the condition required for $L_{M}=L_{\mathrm{T}}$, namely, that of area equivalence. The ideal square spectral bandpass is represented by the left side of Eq. (78) for which the bandpass is from $\lambda_{1}$ to $\lambda_{2}$. The area-equivalent effective bandpass of the nonideal response function is given by the right side of Eq. (78). The nonideal response is said to be equivalent to the ideal response because there is equal area under each curve.

The meaning of such a measurement is limited to the following: "Provided that $L_{\lambda}$ is spectrally uniform, the total radiance has a magnitude given by Eq.(78)." The recommended practice is to 


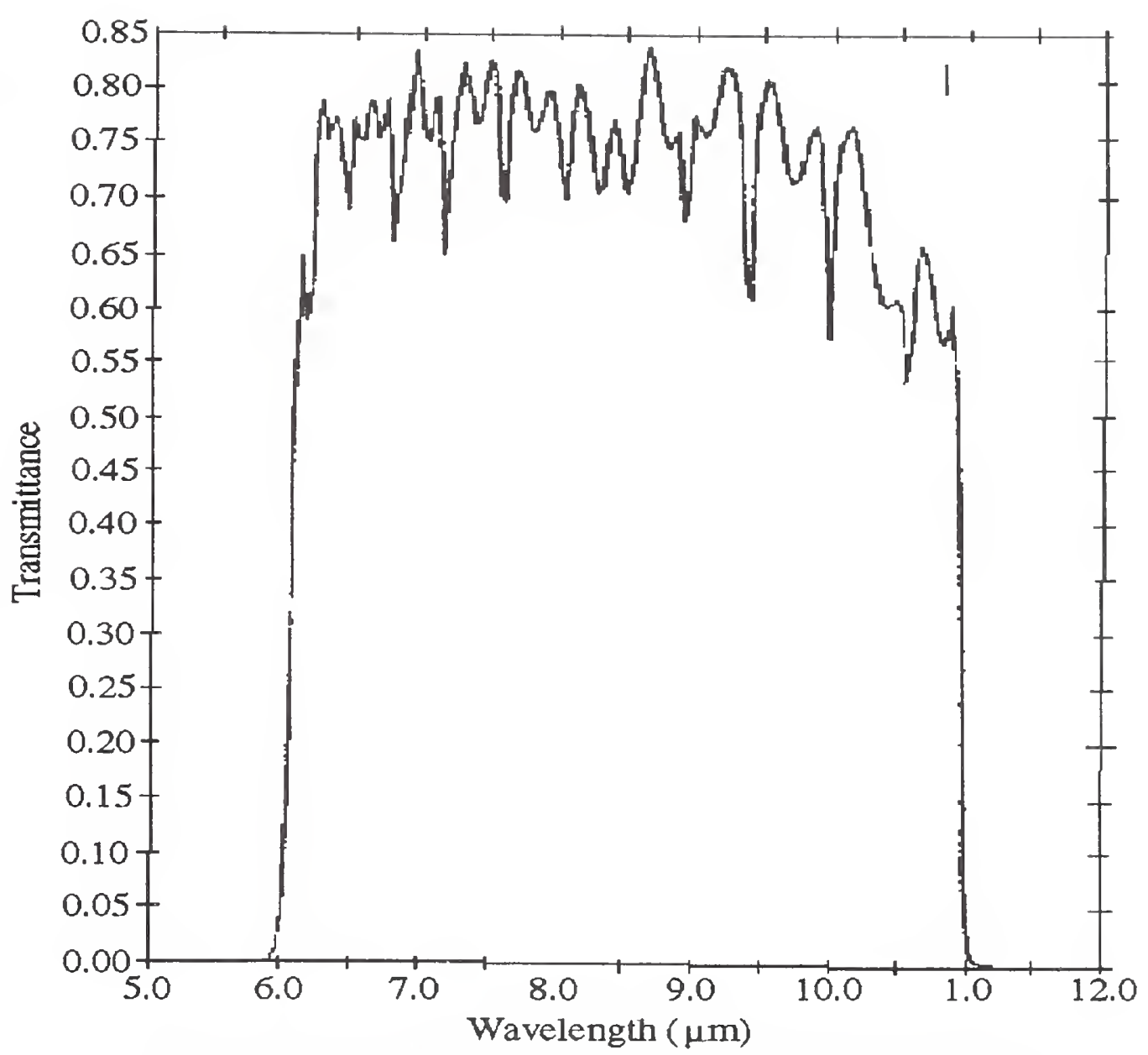

Figure 8. Typical bandpass interference filter transmittance.

calculate the radiance from Eq. (78), which depends upon how the relative spectral response $\rho(\lambda)$ is normalized. It can be normalized to the mean of an "inner-band" or to the peak. In any case the calibration report should indicate the type of normalization used $[14,19]$.

It should also be noted that peak normalization guarantees a bias in the measured radiance in as much as the response over the bandpass is less than, or equal to, the peak response; this means the radiance is underestimated as discussed in Ch. 5, Eq. (5.45) Ref. [9].

Corrections for nonuniform spectral bandpass can be provided if the source spectrum is known from theory or measurement [16]. Equation (76) can be rewritten as 


$$
L_{\mathrm{T}}=\max \left(L_{\lambda}\right) \int_{\lambda_{I}}^{\lambda_{2}} \varphi(\lambda) \mathrm{d} \lambda
$$

where $\max \left(L_{\lambda}\right)$ is the peak of $L_{\lambda}$ with units $\left[\left(\mathrm{W} /\left(\mathrm{m}^{2} \mathrm{sr}\right)\right) / \mu \mathrm{m}\right]$ and $\varphi(\lambda)$ represents the relative spectral radiance [unitless], or the "relative spectrum." Therefore, corrections for nonuniform spectral bandpass can be made upon the basis that $\varphi(\lambda)$ can be independently determined by theory or measurement so that only $\max \left(L_{\lambda}\right)$ need be determined by the radiometric measurement. Then Eq. (77) becomes

$$
r=G A \Omega \max \left\{R_{\mathrm{I}}(\lambda)\right\} \max \left(L_{\lambda}\right) \int_{0}^{\infty} \rho_{1} \varphi(\lambda) \mathrm{d} \lambda
$$

from which $\max \left(L_{\lambda}\right)$ can be obtained for a measurement as

$$
\max \left\{L_{\lambda}\right\}=\frac{r}{G A \Omega \max \left\{R_{\mathrm{I}}(\lambda)\right\} \int_{0}^{\infty} \rho_{\mathrm{I}}(\lambda) \varphi(\lambda) \mathrm{d} \lambda} .
$$

Note that $r$ is obtained from the measurement, $\max \left\{R_{\mathrm{I}}(\lambda)\right\}, \rho_{\mathrm{I}}(\lambda), G$ and $A \Omega$ are obtained from the calibration. Thus $\max \left(L_{\lambda}\right)$ is determined and the total radiance $L_{\mathrm{T}}$ can be calculated using Eq.(81) over any region $\lambda_{1}$ to $\lambda_{2}$ provided $\varphi(\lambda)$ is known over that region.

This discussion leads to the conclusion that the best sensor design is one that incorporates both radiometric and spectrometric measurements.

It is necessary to estimate the uncertainty in the likely event that the source spectrum is unknown and corrections cannot be made. This is difficult because any estimation of uncertainty must be made upon the assumption of some source spectral distribution. A limiting case, namely a single line-source, can be investigated as follows. First, it is necessary to assume that the 
line-source is guaranteed to fall within some nominal band. Then, the mean and variance can be calculated for that band from the measured spectral response data. In this case, the uncertainty is given by

$$
u_{\mathrm{SR}}\left(\bar{\rho}_{1}\right)=\frac{s\left(\bar{\rho}_{1}\right)}{\bar{\rho}_{1}}
$$

where this is defined as the standard uncertainty of the relative spectral response $u_{\mathrm{SR}}\left(\bar{\rho}_{\mathrm{I}}\right)$, which is a Type A component of uncertainty. For example, examination of Figure 8 indicates variations of the order of \pm 20 percent in the spectral transmittance of a typical filter which will be a major contributor to the relative spectral responsivity in a broad band radiometer.

\subsubsection{BAND-TO-BAND UNCERTAINTY}

The band-to-band uncertainty is of interest because certain discrimination algorithms are based upon ratios of irradiances measured in appropriate radiometer bands. The target temperature can be related to a band ratio by Planck's equation, for example. This discussion is applicable to measurements with a spectrometer or a multiband radiometer.

The band-to-band uncertainty can be determined using the same data as obtained for the spectral purity test given in Section 3.2.8. In this case, a series of measurements is obtained where the temperature of a standard blackbody source is varied over the range expected in the measurements. These data are obtained simultaneously in multiple radiometric bands for the radiometer and over the spectrum for the spectrometer. The absolute responsivity is calculated for each band and for each temperature. The ratio of the $i$ th and $j$ th bands is

$$
y_{i j}=R_{i} / R_{j}
$$

which should be invariant over the range of source temperatures in the same pair of bands. The spread in the ratio provides a measure of the band-to-band uncertainty.

The best estimate of the uncertainty of the band ratio measurements is given by the ratio of the 
standard deviation of $y$ to the mean $y$. The standard deviation of $y$ is given in terms of the means, variances, and covariances of $R_{i}$ and $R_{j}$. Thus, the band-to-band standard uncertainty, which is a Type A component, is given by

$$
u_{\mathrm{BB}}\left(y_{i, j}\right)=\frac{s(\bar{y})}{\bar{y}}=\left[\frac{s^{2}\left(\bar{R}_{i}\right)}{\bar{R}_{i}{ }^{2}}+\frac{s^{2}\left(\bar{R}_{j}\right)}{\bar{R}_{j}{ }^{2}}-2 \frac{\operatorname{cov}\left(\bar{R}_{i}, \bar{R}_{j}\right)}{\bar{R}_{i} \bar{R}_{j}}\right]^{1 / 2}
$$

where $\operatorname{cov}\left(R_{i}, R_{j}\right)$ is the covariance between $R_{i}$ and $R_{j}$.

There is a possibility that the variance of $y$ tends to zero if the covariance has the proper magnitude and sign. This would happen if the noise fluctuations were completely correlated so that each erroneous observation of $R_{i}$ would be exactly compensated for by a corresponding erroneous observation of $R_{j}$ [18].

This calculation, estimating the ratio uncertainty, is completed for the multiband radiometer in order to obtain the uncertainty for all possible band ratios. For the spectrometer, the uncertainty is obtained for all sub-bands over the entire measured spectrum as a function of wavelength.

\subsection{PROPAGATION OF UNCERTAINTIES -COMBINED STANDARD UNCERTAINTY}

In general, the ISO standard, Guide to the Expression of Uncertainty in Measurement, commonly called the GUM [5] and NIST guide lines (Appendix 2) recommend the uncertainty of the results of a measurement be expressed as a standard deviation (the positive square root of the variance), see Eq. (41), and be termed the Standard uncertainty. The uncertainty in the result of a measurement generally consists of several components of uncertainty. The term combined standard uncertainty is recommended to be used and that these components be combined, using the law of uncertainty propagation, by the positive square root of the sum of the variances and covariances of these components, see Eq. (43). This is the root-sum-square (RSS) method.

The ISO standard also recommends the use of a coverage factor of 2 , used as a multiplier of the combined uncertainty, in order to obtain an expanded uncertainty in which the uncertainty interval is approximately 95 percent. Although, coverage and expanded uncertainty have not yet been utilized in specifying or qualification testing in the field of radiometry and spectrometry, they 
should be incorporated in such future documentation as the American National Standard Institute (ANSI) officially adopted the ISO standard as the American National Standard. The new standard is ANSI/NCSL Z540-2-1997, and its full title is American National Standard for Expressing Uncertainty-U.S. Guide to the Expression of Uncertainty. Also, the National Conference of Standards Laboratories (NCSL), which has broad representation from U.S. industry incorporated the basic principles of the ISO standard and the NIST guidelines in its Recommended Practice R12, Determining and Reporting Measurement Uncertainties.

\subsubsection{OLD TERMINOLOGY AND RECOMMENDED PRACTICE}

The terms precision and accuracy have been used widely in expressing the uncertainty of measurement results in the literature. There has been quite a variance in the interpretation of these terms and their usage, and NIST guidelines discuss this issue in section D.1 in Appendix 2. However, these concepts have been used in the application of electro-optical sensors with the following definitions. The term precision is defined to include the residual and non-ideal uncertainties besides the uncertainties of repeatability and reproducibility of measurements made with the sensor. The term accuracy is defined to refer to absolute measurements which implies tying the measurements to an absolute scale. For example, the precision of a measurement obtained with a spectrometer deals with the uncertainty of the relative energy in various sub-bands besides the repeatability and reproducibility of those values. However, it is the uncertainty of absolute energy in a sub-band that is of interest in radiometry which is expressed by the term accuracy. In other words, the definition of precision differs from the widely accepted definition discussed in section D.1.2 in Appendix 2 which is just the uncertainty due to repeatability and reproducibility of measurements. Such variation in the usage of the term precision leads to confusion. The same is the case for the definition of accuracy as discussed in section D.1.1.1 in Appendix 2. Therefore, the terms precision and accuracy are considered as old terminology the usage of which is recommended to be replaced with unambiguous terms pre final combined standard uncertainty and combined standard uncertainty respectively. Therefore, the concept of precision in old terminology as defined earlier for sensor system calibrations fits well with the definition of the term pre final combined standard uncertainty as it includes all the uncertainties 
except the uncertainty associated with the absolute standard. Similarly, the concept of accuracy in the old terminology fits well with the term combined standard uncertainty of the sensor performance in the new terminology which is defined as the positive square root of the sum of the variances and covariances of the combined uncertainty of the sensor and the standard source uncertainty. It is to be noted that all the uncertainties referred to in this document are relative to their mean values and expressed as percentages based on Eq. 42 as discussed in section D.1.4 in Appendix 2 .

In dealing with electro-optical systems, the sources of uncertainty inherent in the sensor performance including that of repeatability and reproducibility can be broadly classified as follows:

1. Noise: It is caused by the random variations in sensor response due to photon, thermal, and quantization effects. The noise uncertainty is a repeatability uncertainty and can be made negligibly small by taking sufficient data points in each snapshot as discussed in section 3.2.2. 2. Residual uncertainties: These uncertainties arise from corrections of offset, nonlinearity, and nonuniform response as discussed in sections 3.2 .3 and 3.2.4. Reproducibility variations in sensor response are evaluated as part of residual uncertainties.

3. Uncertainties due to Nonideal response: These uncertainties arise from nonuniform spatial and spectral response of the sensor as discussed in sections 3.2.5, 3.2.6, 3.2.8, 3.2.9, and 3.2.10.

The following are examples of several measurement goals and the associated uncertainty estimates.

Generally an integrating radiometer is utilized to obtain absolute measurements of radiance or irradiance of a target source or background. Two types of integrating radiometers are illustrated here:

The first example, a spatial or imaging radiometer, provides a measure of the energy integrated over a relatively wide spectral band, but provides an image of the target or distribution of the background. The calibration Eq. (33) in section 2.2.1 would be applicable to evaluate the uncertainties. However, it should be noted that the nonlinearity of response in each pixel is evaluated in the case of the array detector as part of its nonuniformity of response as discussed in section 3.2.4. An estimate of the combined standard uncertainty of such a spatial, or imaging, 
radiometer is given by

$$
u_{\mathrm{c}}(L)=\left[u_{\mathrm{FF}}^{2}(\bar{R})+u_{\mathrm{FOV}}{ }^{2}(\bar{\rho})+u_{\mathrm{MTF}}^{2}(\bar{r})+u_{\mathrm{A}}^{2}\left(\bar{R}_{\mathrm{L}}\right)+u_{\mathrm{S}}^{2}(\bar{L})\right]^{1 / 2}
$$

which includes the effects of non-uniformity corrections (NUC) given by the first two terms, modulation transfer function, absolute response (spectral purity), and standard source radiance uncertainty. The image (as opposed to an absolute distribution) may be the only measurement goal of an imaging radiometer and in this case the standard source uncertainty terms would not be included in Eq. 85, and the resultant uncertainty is pre final combined standard uncertainty which is equivalent to precision in the old terminology..

The second example, is that of a spatial and wavelength integrating radiometer, which measures the total radiance integrated over a relatively large spatial (FOV) region and a relatively large spectral bandwidth. Again, Eq. (33) would be applicable to evaluate uncertainties. However, such a radiometer typically uses a light chopper and coherent rectification so that offset and flat-field corrections do not apply; but, uncertainties due to nonlinear response given by Eq. (53), nonuniform spectral response given by Eq. (82), and a nonuniform field-of-view (FOV) response given by Eq. (61) apply. In this case the combined standard uncertainty is given by

$$
u_{\mathrm{c}}(L)=\left[u_{\mathrm{NL}}^{2}(\bar{R})+u_{\mathrm{SR}}^{2}\left(\bar{\rho}_{\mathrm{l}}\right)+u_{F O V}^{2}(\bar{\rho})+u_{\mathrm{A}}^{2}\left(\bar{R}_{\mathrm{L}}\right)+u_{\mathrm{S}}^{2}(\bar{L})\right]^{1 / 2}
$$

which also includes absolute radiance responsivity (spectral purity) given by Eq. (70) and standard source uncertainty given by Eq. (67).

Nonideal spatial and spectral response can be large contributors to radiometric measurement uncertainty; optical designs are available which can result in nearly-ideal field of view response. Nonuniform spectral response uncertainties can be reduced by optimal "square" filter design and flat or uniformly responsive detectors or by accompanying bore-sighted spectrometer measurements as indicated in Section 3.2.9.

A spectrometer is normally used to measure the relative spectral radiance (the spectrum) of a 
source; however, a spectrometer can be calibrated for the absolute spectral radiance in which case it is sometimes referred to as a spectro-radiometer.

The components of uncertainty that should be included in an uncertainty analysis of a spectrometer depend upon the sensor design to a great extent. For example, offset error and nonlinearity correction are not possible for an interferometer (multiplex) spectrometer. On the other hand, the circular variable filter (CVF) (sequential) spectrometer is subject to nonlinearity problems. Nonuniform spectral response uncertainty does not apply to the high resolution spectrometer.

Example 3 given below illustrates the calibration and uncertainty analysis for a CVF spectrometer for relative and standard uncertainties, and for radiance and irradiance sources. 


\section{EXAMPLE 3}

\section{CIRCULAR VARIABLE FILTER (CVF) SPECTRORADIOMETER}

Calibration and uncertainty analysis for a CVF spectroradiometer illustrates most of the calibration and uncertainty aspects of both a radiometer and a spectrometer, point-source (irradiance) and extended-area (radiance). The sensor specifications are given in Example 2.

A spectroradiometer is a spectrometer calibrated for absolute measurements; hence, the term spectroradiometer. Example 2 discussed earlier in Section 2, provided a prediction of performance using the measurement equation. This example illustrates the details of a practical calibration and uncertainty analysis.

\section{Calibration tests.}

The calibration tests to which the CVF spectroradiometer were subjected are as follows:

1. Dark-noise

2. Nonlinearity

3. Spectral scan position

4. Spectral resolution

5. Field of View

6. Extended-area source absolute radiance calibration (spectral purity)

7. Point source absolute irradiance calibration (spectral purity)

This sensor has only one detector; consequently the flat-field correction and associated uncertainty does not apply as it would with a spacial sensor.

\section{Uncertainties}

The uncertainties of interest here are as follows.

1. Noise uncertainty $u_{N}(\bar{r})=1 / S N R$ (See Section 3.2.2) 
2. linearity uncertainty $\pm 5.53 \%$

(See Section3.2.3)

3. Spectral scan position uncertainty $\sim \pm 2 \%$

4. Angular spatial (field of View) responsivity uncertainty $\pm 10.8 \%$

(See Section 3.2.5)

5. Extended-area absolute radiance responsivity (spectral purity) $\sim \pm 6 \%$ at $20.4 \mu \mathrm{m}$ ) (See Section

3.2.8 and Figure 9)

6. Point-source absolute irradiance responsivity (spectral purity) $\sim \pm 3.3 \%$ for $5-22 \mu \mathrm{m}$ worse case (See Section 3.2.8 and Figure 12)

7. Standard source uncertainty

(See Section 3.2.7)

Extended-area radiance source $\sim \pm 4.47 \%$

Point-source irradiance source $\sim \pm 1.67 \%$

Notice that with a chopped system utilizing coherent rectification that an offset error is not significant; consequently no offset correction or associated uncertainty estimate are required. In addition, the nonideal spectral response function uncertainties, as given in Section 3.2.9, do not apply to a high resolution spectrometer.

\section{Test Conditions}

1. Noise and linearity data were obtained with a standard blackbody source, collimator, and precision aperture set. Calibration snapshots (see Section 3.2.1) were obtained over the entire dynamic range by varying the aperture areas for a series of fixed temperature sources. Essentially noise-free response was obtained as the mean of each snapshot in accordance with Eq.(45) for nonlinearity analysis.

Best-fit analysis described in Section 9-3 in Ref. [15] was used to obtain the responsivity correction factor (Eq.(51), Section 3.2.3) and the uncertainty was obtained as the ratio of the standard deviation of the responsivity for the best-fit divided by the mean responsivity for all data points.

2. Spectral Scan position data were obtained with a source, monochromater, and collimator. This 
CVF spectrometer utilizes a 3-segment composite filter.

Segment 1 covers 5.5 to 49.3 percent scan which corresponds to 4.80 to $8.86 \mu \mathrm{m}$.

Segment 2 covers 57 to 74.5 percent scan which corresponds to 8.87 to $13.63 \mu \mathrm{m}$.

Segment 3 covers 80.2 to 100 percent scan which corresponds to 13.7 to $22.85 \mu \mathrm{m}$.

Discontinuities are observed in the response and derived spectral parameters as shown below.

A data-set of wavelength vs. scan position (percent scan) were obtained and best-fit to linear functions for each filter segment. Mean and standard deviation were obtained to provide

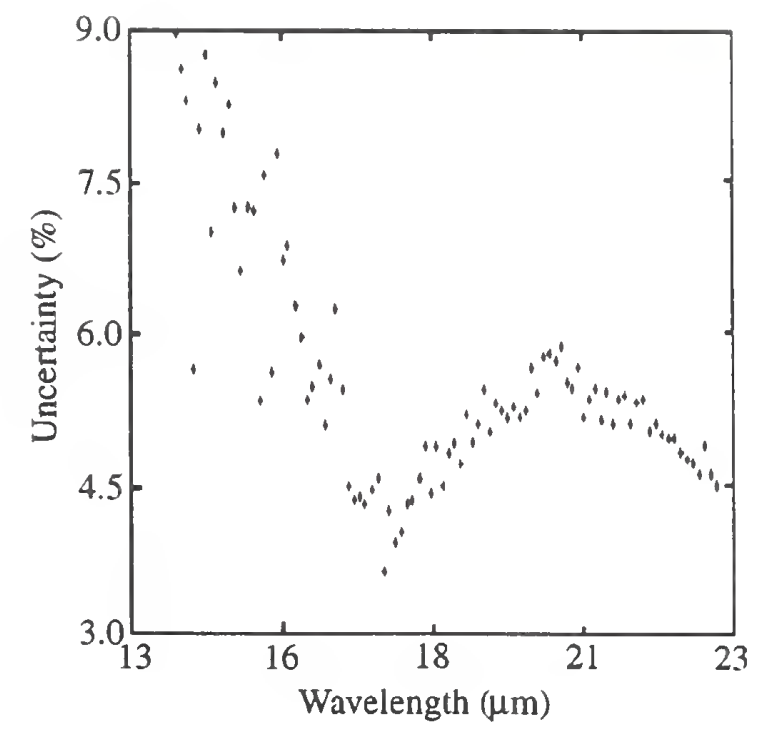

Figure 9. Standard uncertainty of the extended-area source absolute responsivity calibration (1-sigma values given as percentages). corresponding calibration equations and uncertainty for each segment.

3. Spectral resolution data were obtained by stimulating the sensor with a blackbody source filter with narrow band filters, and a polystyrene absorption cell.

4. The angular spatial (field of view) response were obtained as the response to a point source, generated by a pointing mirror collimator, for a $25 \times 25$ matrix. See Section 3.2.5. An inner-core was defined and the inner-core mean response was determined as 0.804 . The uncertainty was estimated for a worse-case assumption that the target is a point source within the inner core. The uncertainty is given by the standard deviation over the mean relative responsivity.

5. Extended-area absolute radiance responsivity (spectral purity) test was obtained with a full-field full-aperture uniform extended-area source (see Section 3.2.7). The response was obtained over the free spectral range (approximately 5 to $22 \mu \mathrm{m}$ ) and over much of the dynamic range for a 
series of source temperatures. The results are presented in Figure 14.

Figure 9 illustrates the percent uncertainty in absolute responsivity over the 7 temperature scans for the third filter segment (13.70 to $22.85 \mu \mathrm{m})$.

Data below $17 \mu \mathrm{m}$ are probably invalid because of poor SNR. Notice that the uncertainty at $20.4 \mu \mathrm{m}$ is about \pm 6 percent.

Figure 10 illustrates the relative spectral response function (instrument function). Note: $\rho_{\mathrm{L}}(\lambda)=1$ at the $20.4 \mu \mathrm{m}$.

6. Point-source is used for absolute irradiance (spectral purity) test. The experimental setup is described in Section 13.6.4. Ref.[15]. It was obtained with a source and a fixed aperture collimator. The response was obtained over the free spectral range (approximately 5 to $22 \mu \mathrm{m}$ ) and over much of the dynamic range for a series of source temperatures.

Figure 11 illustrates the response in volts as a function of scan sample-point (there are 473 sample points per scan) for 10 standard source temperatures: $627 \mathrm{~K}, 682.3 \mathrm{~K}, 777.4 \mathrm{~K}, 926.7 \mathrm{~K}$, $976.6 \mathrm{~K}, 991.6 \mathrm{~K}, 1055.6 \mathrm{~K}, 1134.3 \mathrm{~K}, 1204.5 \mathrm{~K}$, and $1259.8 \mathrm{~K}$. The following are noted: Zero

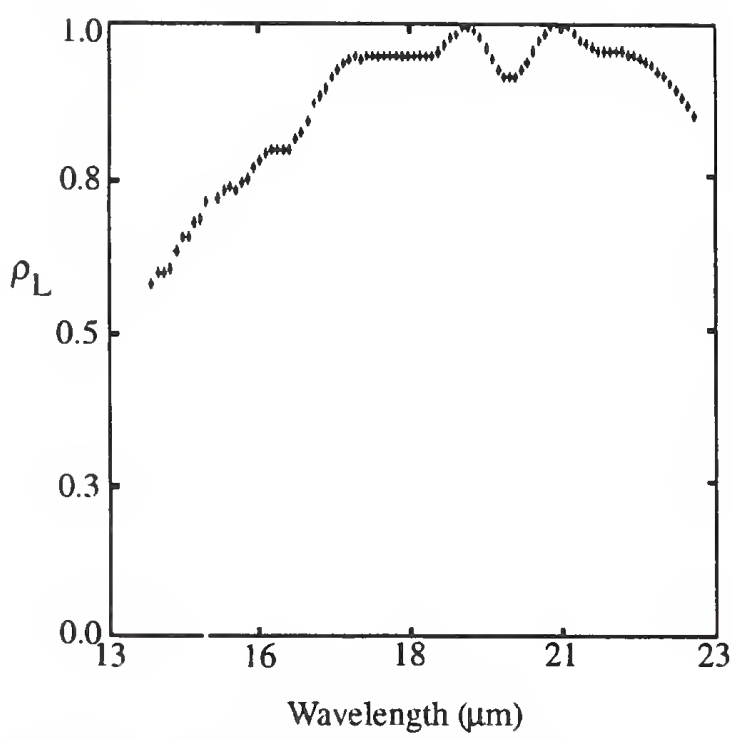

Figure 10. Relative spectral response $\rho_{\mathrm{L}}(\lambda)$ or "instrument function" for the extended-area source absolute calibration.

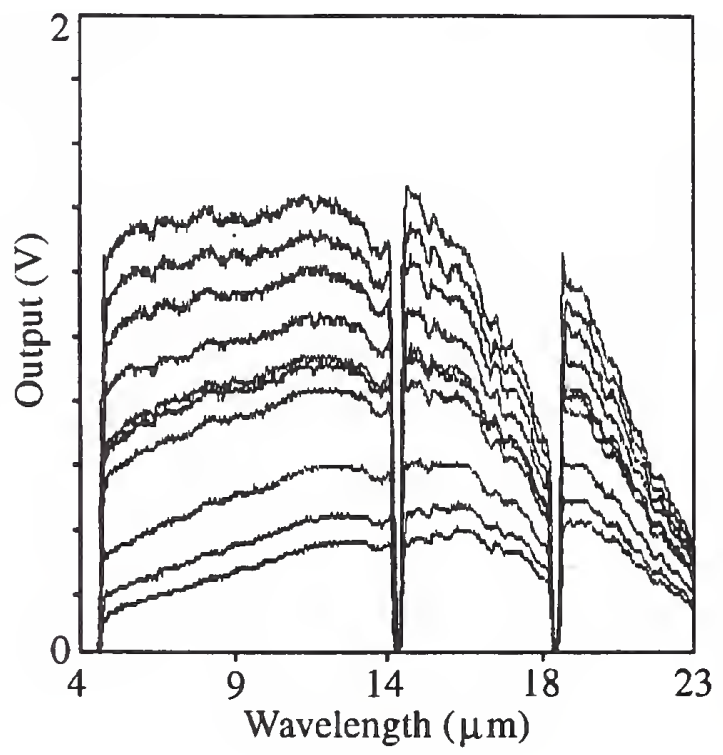

Figure 11. Scans obtained for the pointsource absolute responsivity calibration. response at the filter segment masks clearly mark the masked filter joints. A relatively large discontinuity in response between filter segments exists for the same wavelength. Greater energy and response at short wavelengths for higher source 
temperatures is noted.

Figure 12 illustrates the percent uncertainty in absolute irradiance responsivity over the 10 temperature scans and over the free spectral range. Note the uncertainty varies between \pm 2.5 and \pm 3.3 percent with wavelength.

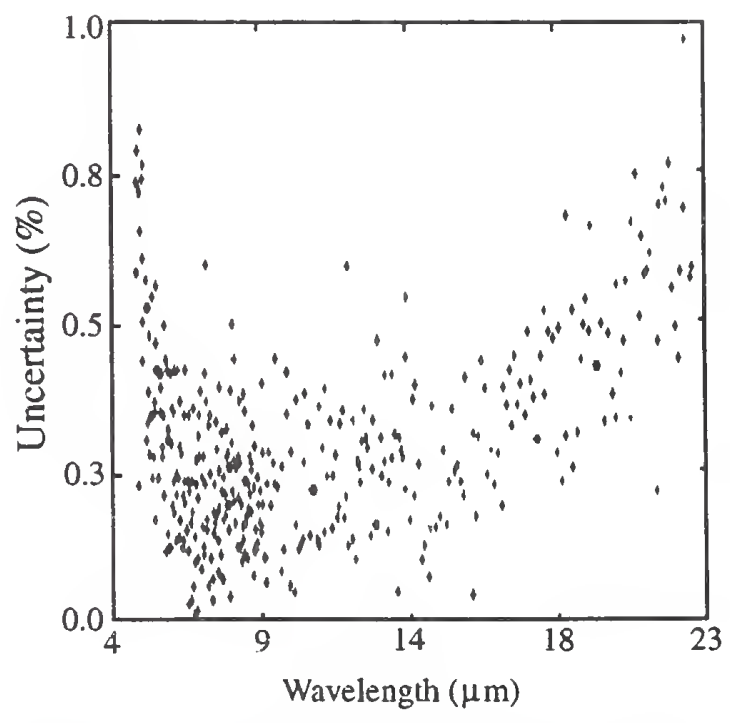

Figure 12. Standard uncertainty of the point-source absolute irradiance responsivity calibration (1-sigma values given in percentages). 


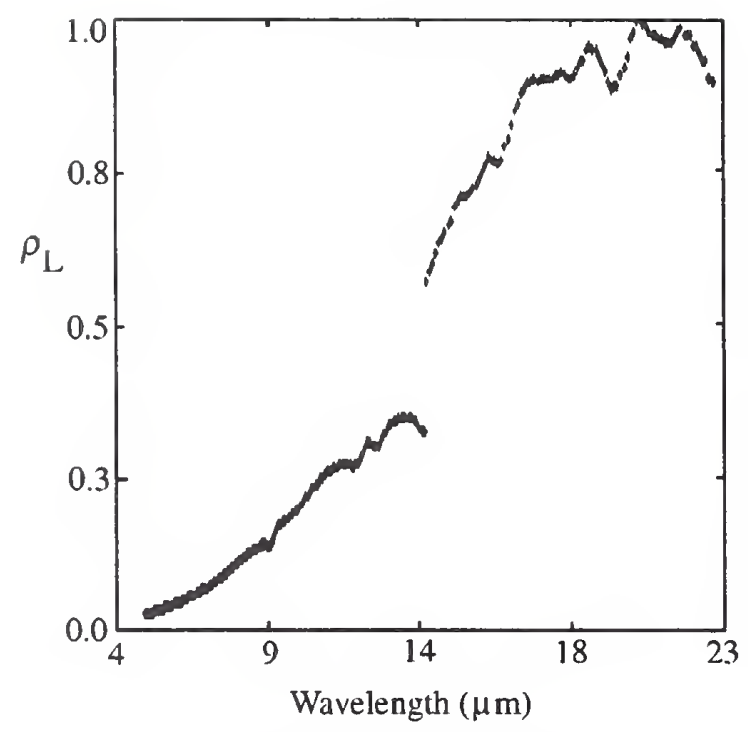

Figure 13. Relative spectral response $\rho_{\mathrm{L}}(\lambda)$ or "instrument function" for the point-source absolute irradiance calibration.

Figure 13 illustrates the relative spectral response function (instrument function). Note that $\rho_{\mathrm{L}}(\lambda)=1$ at the $20.4 \mu \mathrm{m}$. Note in particular the discontinuity in the instrument function at the mask about $13.6 \mu \mathrm{m}$. This compensates for variations in transmittance of the second and third filter segments.

7. The uncertainty in the radiance resulting from a temperature uncertainty can be determined by using [Eqs. (64), (65), (66) and (67)] given in Section 3.2.7. In addition to temperature uncertainties, it is necessary to take into account the emissivity uncertainty of the large area (extended-area) source which is estimated at \pm 2.0 percent.

The following tabulation provides standard source uncertainties: 
STANDARD SOURCE UNCERTAINTIES

Temperatures $[\mathrm{K}] \quad$ Wavelength $[\mu \mathrm{m}] \quad$ Uncertainty $[\%]$

\begin{tabular}{|c|c|c|}
\hline $71^{*}$ & 20.4 & 4.47 \\
\hline 83 & 20.4 & 2.96 \\
\hline 95 & 20.4 & 3.01 \\
\hline $627 * *$ & 5 & 1.67 \\
\hline 944 & 5 & 0.71 \\
\hline 1260 & 5 & 0.38 \\
\hline 627 & 10 & 0.76 \\
\hline 944 & 10 & 0.29 \\
\hline 1260 & 10 & 0.14 \\
\hline 627 & 22 & 0.25 \\
\hline 944 & 22 & 0.08 \\
\hline 1260 & 22 & 0.04 \\
\hline
\end{tabular}

* Data for the extended area source including emissivity uncertainty and for a temperature uncertainty of 0.5 degree. ${ }^{*}$ Data for the source for a temperature uncertainty of 4 degrees.

The above tabulation gives the radiance uncertainty over the range of temperatures used in the absolute (spectral purity) calibration and over the range of wavelength used.

\section{Discussion}

A cardinal rule of calibration is that one should calibrate a sensor under the same conditions for 
which it is to be used. This suggests that the absolute radiance calibration should be obtained with an extended-area source. However, the low-temperature extended-area source has a wavelength distribution which is nearly a step-function; that is, there is no response below about $15 \mu \mathrm{m}$ when the source temperature is reduced such that the sensor is not saturated at $20 \mu \mathrm{m}$. Figure 14 illustrates the response in volts as a function of scan sample-point (there are 473 sample points (sub-bands) per scan) for 10 standard source temperatures of which 7 are identified from the top down: $94.7 \mathrm{~K}, 90.3 \mathrm{~K}, 85.2 \mathrm{~K}, 81.6 \mathrm{~K}, 78.2 \mathrm{~K}, 74.4 \mathrm{~K}, 70.8$. K. Note the rapid fall off in energy and response at short wavelengths.

The flux must be greatly attenuated to permit using higher temperature sources to achieve a more uniform response over the entire spectral range. A collimator used with a very small aperture, a neutral density filter, or a combination of collimator and filter can be used to provide the necessary attenuation. However, the uncertainties are difficult to evaluate for the collimator and require a knowledge of the uncertainty of the aperture area, the collimator focal length, scattering effects, and mirror reflectance. The use of a neutral density filter provides added uncertainties which are a function of wavelength.

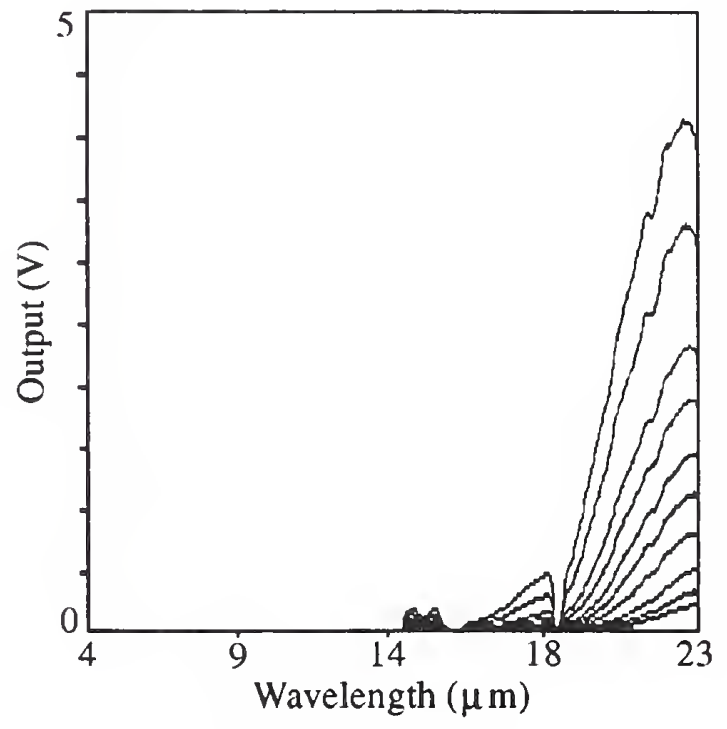

Figure 14. Scans obtained for the extended-area source absolute responsivity calibration.

These problems are resolved by using the collimator and small aperture (which are less likely to be wavelength dependent) with a point source to determine the instrument function (See Section 3.2.8) which is the relative spectral responsivity shown in Figurel3 over the free spectral range of the spectrometer. Note: Figure10 and Figure13 overlap for the wavelengths covered. However, Figure 13 obtained by the point source absolute irradiance test covers the full spectral range. The extended area source calibration at the wavelength of maximum responsivity $-20.4 \mu \mathrm{m}$. is used to "anchor" the instrument function to obtain an absolute radiance calibration of the spectrometer. 
The point-source calibration can be used to provide an absolute irradiance calibration of the spectrometer. The uncertainty in the absolute irradiance responsivity calibration is shown in Figure

12. This uncertainty also reflects in the instrument function as discussed in Section 3.2.8.

\section{Uncertainty analysis}

It is recommended that the uncertainty be evaluated for a specified SNR for both writing of sensor specifications and qualification testing. This is most appropriately done for infinite SNR. This provides an optimistic uncertainty characterization that depends only upon sensor residual uncertainties (nonlinear correction, etc.) and nonideal uncertainties (nonideal spectral and spatial response). Thus, the uncertainty estimates given below neglect the effects of noise, i.e. the SNR is assumed to be infinite.

It should be noted that source uncertainties and absolute calibration uncertainties vary with wavelength; in the calculations given below, uncertainties are given for the measurement of radiance and irradiance based upon the worse case performance of the CVF spectroradiometer at a particular wavelength setting. In addition, there is an intrinsic wavelength uncertainty of $\pm 2 \%$ according to the characterization data given earlier.

\section{Point source uncertainty}

An estimate of the pre final combined standard uncertainty of a measurement of irradiance is given for the components of uncertainty given above by

$$
u_{\mathrm{p}}(E)=\left[u_{\mathrm{NL}}^{2}(\bar{R})+u_{\mathrm{A}}^{2}\left(\bar{R}_{\mathrm{E}}\right)+u_{\mathrm{FOV}}^{2}(\bar{\rho})\right]^{1 / 2}= \pm 12.6 \%
$$

for large SNR which includes the nonlinearity 5.53\%, absolute irradiance responsivity (pointsource) $3.3 \%$, and the angular (field-of-view) spatial uncertainties $10.8 \%$, the latter of which dominate in this case. Therefore, the pre final expanded uncertainty, $U_{\mathrm{p}}(E)=2 u_{\mathrm{p}}(E)$ will be \pm $25 \%$ as discussed in section 3.1 . 
The combined standard uncertainty of a measurement of irradiance is given by

$$
u_{\mathrm{c}}(E)=\left[u_{\mathrm{p}}^{2}(E)+u_{\mathrm{s}}^{2}(\bar{L})\right]^{1 / 2}= \pm 12.7 \%
$$

for large SNR which includes the relative combined standard uncertainty and of the standard source uncertainty $1.67 \%$. Therefore, the expanded standard uncertainty, $U_{\mathrm{c}}(E)=2 u_{\mathrm{c}}(E)$ will be $\pm 25 \%$.

\section{Extended-area uncertainty}

An estimate of the pre final combined standard uncertainty of a measurement of radiance is obtained for the components of uncertainty given above by

$$
u_{\mathrm{p}}(L)=\left[u_{\mathrm{NL}}^{2}(\bar{R})+u_{\mathrm{A}}^{2}\left(\bar{R}_{\mathrm{E}}\right)+u_{\mathrm{A}}^{2}\left(\bar{R}_{\mathrm{L}}\right)\right]^{1 / 2}= \pm 8.8 \%
$$

for large SNR which includes the nonlinearity $5.53 \%$, point source absolute irradiance responsivity uncertainty(used for the instrument function over 5 to $22 \mu \mathrm{m}$ ) 3.3\%, and extendedarea absolute radiance responsivity uncertainty (used to anchor the instrument function at 20.4 $\mu \mathrm{m}) 6 \%$. Therefore, the pre final expanded uncertainty $U \mathrm{p}=2 u_{\mathrm{p}}(L)$ will be $\pm 18 \%$ as discussed in section 3.1. The combined standard uncertainty of a measurement of radiance is given by

$$
u_{\mathrm{c}}(L)=\left[u_{\mathrm{p}}^{2}(\bar{L})+u_{\mathrm{s}}^{2}(\bar{L})\right]^{1 / 2}= \pm 9.9 \%
$$

for large SNR which includes the relative combined standard uncertainty $8.8 \%$ and the standard source uncertainty $4.4 \%$. Therefore, the expanded uncertainty, $U_{c}=2 u_{c}(L)$ will be $\pm 20 \%$.

As an epilogue, we might point out that the uncertainty analysis for remote sensing sensors is rather complex and needs to be carefully defined and documented as recommended here. Also, the uncertainties for IR sensors as shown in the example 3 are in general large compared to the 
desired goals and the challenge for the calibration community at present is to bring these combined standard uncertainties for IR sensors to single digit levels to meet various science objectives.

\section{REFERENCES}

[1] A.C. Parr, "A National Measurement System for Radiometry, Photometry, and Pyrometry Based upon Absolute Detectors," NIST Technical Note 1421, U.S. Government Printing Office, Washington, DC 20402-9325, 1996.

[2] "Quantities and units- Part 6: Light and related electro-magnetic radiations," ISO STANDARDS HANDBOOK, QUANTITIES AND UNITS, UDC 389.15, Third edition, 1993, pp. 121-146.

[3] "International Lighting Vocabulary," 3rd Ed., Publ. CIE No. 17(E-11), common to the $\mathrm{CIE}$ and IEC, International Electrotechnical Commission (IEC), International Commission on Illumination (CIE). Bur. Cent. CIE, Paris 1970.

[4] B.N. Taylor, Guide for the use of the International System of Units (SI)," NIST Special Publication 811, U.S. Government Printing Office, Washington, DC 20402-9325,1995.

[5] "Guide to the Expression of Uncertainty in Measurement," ISO/TAG 4/WG 3: International Organization for Standardization, Geneva, Switzerland, 1993.

[6] B.N. Taylor and C.E. Kuyatt, "Guide for Evaluating and Expressing the Uncertainty of NIST Measurement Results," NIST Technical Note 1279, January 1993.

[7] "Quantities and units- Part 0: General Principles," ISO STANDARDS HANDBOOK, QUANTITIES AND UNITS, UDC 389.15, Third edition, 1993, p. 5. 
[8] Reference 6 above, pp. 11-13.

[9] F.E. Nicodemus, "Self-Study Manual on Optical Radiation Measurements," NBS Technical Note 910-1, 910-2 \& 910-4 Ch. 1 to 8 (1976), U.S. Government Printing Office, Washington, DC 20402-9325. On a limited basis these documents are available from the Optical Technology Division, NIST, Gaithersburg, MD 20899-0001

[10] C.L. Wyatt, "Electro-optical System Design," McGraw-Hill Incorporated, NY, 1991, Ch. 8, pp. 111-122

[11] H.J. Kostkowski, "Reliable Spectroradiometry," Spectroradiometry Consulting, La Plata, MD 1997, p. 6.

[12] J.B.Shumaker, "Self-Study Manual on Optical Radiation Measurements," NBS Technical Note 910-7, Ch. 11 (1984), U.S. Government Printing Office, Washington, DC 20402-9325. On a limited basis these documents are available from the Optical Technology Division, NIST, Gaithersburg, MD 20899-0001;

[13] F.E. Nicodemus, "Radiometry." In "Optical Instrument," Part 1 (R. Kingslake, ed.), Applied Optics and Optical Engineering, Vol. 4, Ch. 8. Academic Press, New York, 1967.

[14] F.E. Nicodemus, "Normalization in Radiometry," Appl. Opt. 12, 1973, pp. 2960-2973.

[15] C.L. Wyatt, "Radiometric Calibration: Theory and Methods," Academic Press, New York, N.Y., 1978, pp. 119-125

[16] R.B. Abernethy and B. Ringhiser, "The History and Statistical Development of the New ASME/SAE/AIAA/ISO Measurement Uncertainty Methodology," AIAA/SAE/ASME/ASEE 21 st Joint Propulsion Conference. Monterey, CA., July 8-10, 1985, p. 1. 
[17] D.A. Scribner, K.A. Sarkady, J.T. Caulfield, M.R. Kruer, G. Katz, and C.J. Gridley, "Nonuniformity Correction for Staring IR Focal Plane Arrays using Scene-based Techniques," Infrared Detectors, Focal-plane Arrays, SPIE, Vol. 1308, 1990, pp. 224-234.

[18] Philip R. Bevington, "Data Reduction and Error Analysis for the Physical Sciences," McGraw-Hill Book Co., N.Y., 1969, pp. 56-65.

[19] F.E. Nicodemus and G.J. Zissis, "Report of BAMIRAC-Methods of Radiometric Calibration," ARPA Contract No. SD-91, Rep. No. 4613-20-R (DDC No. AD-289, 375), University of Michigan, Infrared Lab., Ann Arbor, Michigan, 1962, p. 15. 



\section{APPENDIX 1}

RECENT HISTORICAL RECORD THAT LED TO THIS DOCUMENT PREPARATION. 



\section{Dear Colleage:}

The Sensing Systems Working Group of the AlAA Space-Based Observation Systems Committee on Standards (SBOS COS) has initiated an effort to address the concern that many and diverse approaches are utilized to determine the characteristics and performance of space-borne remote sensing systems. To begin to address the issue of comparability among data sets obtained from multiple space-borne sensors, the Sensing Systems Working Group is forming task teams to evaluate the need for sensor system calibration standards, practices or guidelines. The purpose of this letter is to provide you with information on the approach being taken to the issue of space-borne sensing systems calibration standards and to solicit your participation on a task team to specifically focus on visible to short-wave infrared sensor calibration (.5 $\mu \mathrm{m}$ to $2.5 \mu \mathrm{m})$.

A brief overview on "AIAA Activities in Calibration Standards" by Edward W. Koenig is enclosed for your information. This paper describes the rationale for the SBOS COS, the efforts on sensor calibration and the relationship between the COS and the American National Standards Institute (ANSI).

The Visible-SWIR sensor task team will meet for the first time at the upcoming AIAA Annual Conference to be held September $23-25,1992$ at the Hyatt Hotel, Washington, DC, on the "Importance of Standards to Mission Success". Subsequent meetings are planned to be held: January $11-13,1992$ in conjunction with the 31st Aerospace Science Meeting in Reno, Nevada, on "Sensor Calibration"; May 19-21, 1993 at the Hyatt Crystal City (two weeks after the AIAA annual meeting on "Interoperability in Command and Control"); and September/October (TBD) 1993 in conjunction with the Computing in Aerospace meeting to be held in Albuquerque, New Mexico, "Data Comparison".

The objectives of the first Visible-SWIR task team meeting will be to formulate and agree upon a strategy for addressing the most important aspects of calibration of space-borne remote sensing instruments, to plan the scope of the following workshops and to define the product(s) to be produced by the team.

A tentative agenda for our first meeting is as follows:

1. Identification and discussion of Visible-SWIR calibration issues and problems.

2. Define focus of team effort and select highest priority issues to be addressed. 
3. Develop a strategy to understand, define and resolve issues.

4. Planning of future workshops.

5. Assignment of responsibilities.

A listing of top-level discussion topics for you to consider prior to our first meeting includes:

1. Detector/focal plane characterization and calibration.

2. Optical system calibration.

3. Optical filter characterization and calibration.

4. Focal plane/signal processing performance characterization.

5. Sensor system calibration.

6. Reference sources and standards.

7. Radiometric response characterization.

8. In-flight calibration.

9 Reproducibility of tests and test configurations.

10. Intercomparison of sensor systems.

11. Long term stability and knowledge.

12. Environmental effects on system performance and calibration.

If you are interested in participating in the Visible-SWIR calibration team effort, please contact me.

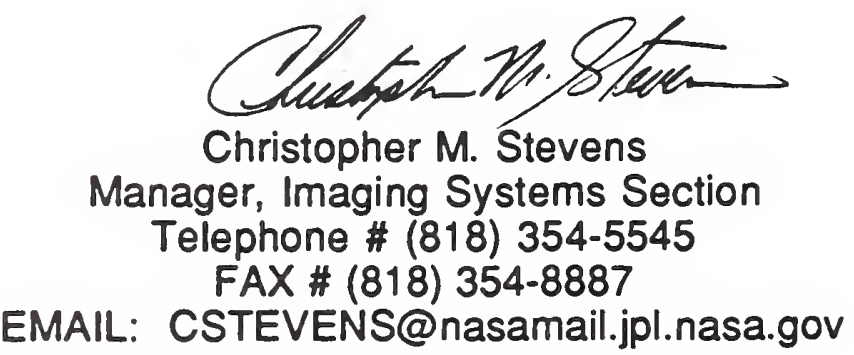

CMS:mdd 
Presented to 2nd Annual SD/USU Symposium on Cryogenic Infrared

Radiometric Sensor Calibration, Sept. 18-20, 1991, Logan UT

American Institute of Aeronautics and Astronautics (AIAA) Activities in Calibration Standards

Edward W. Koenig *

Sensing Systems Working Group Chairman

AIAA-SBOS COS

\section{INTRODUCTION}

The American Institute of Aeronautics and Astronautics (AIAA) initiated a special task group to specifically address the problems and advances in spaceborne observation systems. The AIAA Space-Based Observation Systems Committee on Standards (SBOS COS) was brought into being in 1988 as a means of providing a source of information relating to many of the issues in the areas of Sensing Systems, Communications and Data, Operations, Software Reliability, Spacecraft and Liaison. Working Groups within the COS place emphasis on tasks which are limiting the accuracy, efficiency or other significant qualities of the systems. As long-term activities the tasks were selected for their generic ability to aid future systems, not expecting impact on near term system design or present contractual work in progress.

In the Sensing Systems Working Group the initial concern was for the widely diverse approaches being taken in the definition, performance and evaluation of sensor systems. The first task taken was to develop a set of definitions and physical constants related to spaceborne sensors. This effort has continued, and is being merged with the present tasks, which are to establish calibration standards, guidelines or recommended practices. This has now resulted in the formation of two task teams within the working group and plans are being formulated for an additional four task

„Edward Koenig - a staff engineer with ITT Aerospace/Communications Division in Fort Wayne, IN 
teams. The approach is to separate the tasks by spectral bands since the details of detection, test and evaluation are so much different for various ranges of the spectrum. We have selected the following spectral bands as the general limits for initial consideration.

Passive Sensors

Ultraviolet

Solar

Infrared

Microwave
Active Sensors

Optical

Microwave

The activities of these task groups are expected to lead to a better definition of sensor characterization and performance evaluation as well as overcome major differences in sensor calibration. The results will be coming from a diverse group of persons, not specifically committed to a given program or project as is often the case.

\section{ROLE OF THE AIAA}

The role of the AIAA Standards Program is to ensure the continued enhancement of aerospace industry-wide efficiency and productivity. This is to be achieved by the development of scientific and technical standards document where a need has been identified. The purpose is to pursue standards to be approved as American National Standards. One objective is to have AIAA Standards documents adopted by the Department of Defense- and be listed-d in the DoD Index of specifications and Standards (DODISS) whenever this is appropriate. The AIAA Standards program consists of the development of and publication of scientific and technical standards and data. The technical scope of these standards documents covers all areas of interest to AIAA Technical Committees, including systems, components, materials, products, technologies, methods and practices in aerospace applications. Standards may cover such topics as health, safety, design, testing, construction, maintenance, performance, environment, operation of aerospace devices, equipment, and methods. Published standards documents are of four kinds: Standards, Recommended Practices, Guides, and Special Project Reports. 
The AIAA will cooperate and coordinate with all other competent standards bodies worldwide in the technical areas within the scope of the AIAA Standards Program. Consensus is used as the tool to reach agreement on any given item. A process is in place for review and approval by the individuals, consultants, committees and working groups to which the document is presented. Publication means that the document achieved consensus of the community which typically consists of representatives from government, industry, and academia. Special Project Reports may be published without the consensus procedure. The use of AIAA Standards is entirely voluntary, there is no inherent commitment for any user to adhere to any standards report. Compliance with AIAA Standards documents does not guarantee quality or satisfactory application.

The AIAA will not engage in testing or certification of products, systems, or any other device or method, for demonstrating compliance with AIAA's Standards Documents. It will not consider any patents which may apply to the subject matter of a report or standard. Prospective users are responsible for protecting themselves against liabilities or infringement of patents or copyrights. No individual, group, or committee, whether members of AIAA or not, is authorized to express opinions or act in any way in these matters in the name of AIAA or on behalf of AIAA. The AIAA assumes no responsibility for the use or interpretation of its standards documents. Standards documents follow the same pattern of the AIAA published documents which comprise Standards, Recommended Practices, Guides, and Special Project Reports.

A Committee on Standards (COS) for a specific discipline is directly responsible to STC and shall 1) Develop standards within its scope, 2) Maintain standards within its scope, with review every five years, 3) Achieve a consensus on action proposed, 4) Ensure that duplication of standards is minimized, 6) Advise on standards within its scope, 7) Report the status of standards at least annually to the STC, 8) Document discussions on developing or revising standards and send reports to the Administrator

American National Standards Institute (ANSI)

The American National Standards Institute has been the cornerstone of U S activities for 
voluntary standards ANSI is a private, nonprofit organization with a broad membership of about 1,000 companies, $30 \mathrm{U}$ S government agencies, 250 professional, technical, and trade societies, and labor and consumer groups Our SBOS COS, through the AIAA, is an accredited standards organization of ANSI, which approves American National Standards and represents U S interests in forums such as the International Organization for Standardization (ISO) After a working group develops a document it goes through the SBOS COS approval and AIAA approval, then is submitted to ANSI for approval After ANSI approval AIAA will publish the standard with an ANSI number

Space-Based Observation Systems Committee on Standards The Space-Based Observation Systems Committee on Standards (SBOS COS) is one of these AIAA sponsored committees It was established to specifically look at the problems facing the general field of new and developing spacecraft to prepare for the use of standards, wherever appropriate and beneficial These standards, if extended to a number of spacecraft and multiplicity of similar instruments, will reduce the complexity of assembly and operation, and improve utilization of the data from these systems The charter of the SBOS-COS is the "Adoption of, modification, development, and recommendation of standards, recommended practices, and guidelines relating to satellite subsystems (space segment and ground segment) that study the earth and its environment in space with the following specific requirements:

Specific to space-based observation disciplines

Applicable to multiple programs and/or missions

Economically viable, with emphasis on life-cycle cost savings

Must include recommended method of implementation

Must have clearly defined benefits to multiple contractors,

Government agencies, and users, and

Coordinated with relevant National and international activities

As such the AIAA SBOS COS which is chaired by Mrs. Andrea F. Sebera, previously of Space Systems/Loral and Dr. Neville Marzwell of NASA/Jet Propulsion Laboratory in Pasadena as Vice 
Chair has been active in the formation of working groups with individual responsibilities in the areas of:

Technical Coordination; chairman Lawrence M.G. Enomoto, Esq., which completed a comprehensive document "1991 Earth Observations Directory: A Worldwide Listing of Government Institutions and Related Groups" under the technical leadership of Dr. Paul Uhlir. This committee will continue to coordinate US and international information to aid interface within the observation satellite community.

Communications and Data Systems; Chairman Charles Baird, has task groups working on a Security and Privacy Project, Upper Layers Project, Tools for Communications Systems, Command and Controls Project and Data Compression Standards. Details of these projects, as with others in the COS are described in the SBOS Orbiter, the newsletter of the COS.

Space Operations Working Group; chairman George C. Jackson, developed a Guideline for Human Computer Interfaces and is working on a Guideline for Satellite Autonomy.

Sensing Systems Working Group; chairman Edward W. Koenig; developing a document on Space-based Sensor Systems Terms and Physical Constants, and setting up task groups on calibration as described earlier.

Software Reliability Working Group; chairman Ted Keller, is working on a Standardized Software Reliability Model as a recommended practice.

Spacecraft Working Group; chairman Dr. Janet Bare is working on interfaces, components, subsystems, spacecraft buses, testing and calibration, particularly focusing on small satellites. A database of Characteristics of Satellites and Payloads has been developed and is now available in preliminary form. 
The SBOS COS has just completed a joint NASA/NASDA/AIAA meeting in Tsukuba, Japan, June 26-28, 1991 to discuss international cooperation efforts in Earth Observing Missions. The results were encouraging in having the opportunity to discuss detailed common needs and approaches to improved international communication on interfaces and standards.

The next SBOS COS quarterly meeting will be held in Huntsville, Alabama, from November 13 to 15 and will be dedicated to Extra-terrestrial Exploration. The follow-on meeting will be in the Jet Propulsion Laboratory on February 5 to 7, 1992, and will be dedicated to Autonomous Light Spacecraft.

The AIAA SBOS COS is firmly committed to the stand that U.S. industry needs to be involved in standards development to assure continuous leadership in space-based observation systems.

\section{A Perspective on Calibration}

In the efforts to review and evaluate the needs of the sensor community in the area of calibration it became clear that a number of factors and requirements were emerging which need to be addressed in the calibration of instruments. The needs are seen as:

Definitions: The selection and definition of terms used specifically in sensor systems needs to be improved. An example of an attempt to improve this situation is the report by Dr. Wyatt on the definition and application of accuracy and precision to infrared systems. The need for definitions which stand up to examination and test and the selection of terms for universal use in the industry is very important. Each of the task teams will establish the terms for their particular part of the state of the art and have them reviewed by the SBOS COS to correlate with other groups and with a set of terms already in review by the committee.

Pre-flight calibration sources: The design of calibration sources should follow guidelines established by the most experienced users. Designs must include consideration for uniformity, blackness, thermal stability, thermal sensor placement, control and cleanliness. The method of 
establishing the accuracy of calibration target temperatures must include use of calibrated thermistors or platinum resistance thermometers (PRT) having direct transfer from basic standards. The circuits used to measure these resistances for data collection should also be tested for stability and accuracy. The use of common interface circuits having stability, accuracy and noise rejection should be considered. When possible, providing comparison with other targets or standards will verify the quality of the calibration. An approach used in the calibration of visible channels of the NOAA series of instruments is to use a common calibration target for all instruments. This is a well characterized standard calibration target moved around within the test facility. This general technique could be applied to infrared targets where comparison with the special unit would provide the continuity of standard calibration. It might be possible to use a traveling standard source for final calibration. In this regard there is a real need for a common source for comparative tests, such as a facility where targets may be taken for tests of emissivity, uniformity, temperature accuracy, and stability. If we are to ever relate instruments of different types on a given spacecraft and instruments of similar types on a series of spacecraft we must have a more common technique for calibrating the variety of targets.

Evaluation techniques: The methods of collection of data, calculation of accuracy, precision, non-linearity, stability, background noise, system noise, dynamic range, and other characterizations of instruments varies greatly from laboratory to laboratory. The use of a common approach to the definitions, algorithms, characteristic qualities and even the method of reporting the output of the calibration would be beneficial for all.

Construction of reference targets: The method of construction of infrared calibration targets varies widely. Obviously the instrument requirements set the size, and the mounting configuration sets many of the physical characteristics. For static (staring) tests the use of a round reference surface surrounded by a wall that is as deep as the width of the target is typically used. All surfaces are covered with honeycomb and painted black. The combination of paint emissivity, honeycomb dimensions and wall depth determine the (non-blackness) of the target. A good target will have an effective emissivity of over $99 \%$. The use of common paints, cell dimensions and 
depth-to-width ratios will help provide commonality between instrument calibrations. All of this combines to determine the radiometric error contributed by the target and is a part of the absolute radiometric accuracy definition for a given instrument.

In-orbit calibration: Most meteorological and other science oriented satellites use a blackbody reference at a known temperature and space as a two point calibration of the slope (radiance per count) and offset (counts at zero radiance). This has been adequate for many missions but is not sufficient when non-linearity of the sensor or potential changes in spectral characteristics are necessary to characterize the system. In general the use of a black honeycomb surface on the body of the instrument, viewed through the complete optic system, including the scan mirror, provided a suitable IR source. A full view of space provided the cold reference. Now, with large area arrays, varieties of spectrometers and specialty instruments the needs for spectral calibration, linearity, and stability are expanding the requirements and sophistication of on-board calibration targets and procedures. The problem of a truly accurate and stable solar spectrum calibration source is still with us, with several approaches being reviewed for the EOS program.

Long term calibration: The international community is searching for means of using earth targets and space targets as long term calibration sources for a large variety of instruments. Studies of White Sands, various deserts, coastal areas, clouds, moon, stars and the sun as sources is continuing to define common sources for inter-calibration of instruments and for detection of long term degradation of sensors. In general the limitations of ground targets to moisture, sun angles, area size, spectral definition, atmospheric interference effects and other factors has limited the estimated accuracy to the order of $10 \%$. This is not good enough for the levels of accuracy required for most scientific oriented sensors. The dependence on in-orbit targets will therefore continue.

Data retrieval and comparison: When a group of data sets are collected form various instruments with a common goal the data most likely will require reformatting, recalibration, annotation, and be changed to a different computer language and printed differently in order to compare results. 
The need exists for some standard approaches to identifiers, format, references, and database to permit easier distribution and use of the data. The problems faced by the Earth Observation Systems Data Information System (EOSDIS) in collecting data from historical files, present and future files and presenting a format for user evaluation are enormous. We should probably use the EOSDIS as the baseline for setting guidelines for all data collection and distribution systems.

\section{CONCLUSIONS}

The sensor development community needs to be aware of the activities of the AIAA and ANSI in the preparation of standards, guidelines and recommended practices and the procedures to generate and obtain them. The ANSI standards provide a common denominator of government, industry and international scope to bring the community together in its methodology in design, interfaces, operation and utilization of sensor systems.

We tend to become engrossed in our own portion of a project or technology and develop our own approach to calibration, evaluation and dissemination. The use of guidelines for many of these tasks will cause some added effort in the preparation of test methods but will make the results more universal and aid the whole community over a long period.

Calibration of sensors is a complex and demanding task. Diligence in establishing scientific validity to each part of the test is important. It is also important to prepare for the calibration and to present the data using the best knowledge and experience of the world's experts. In the establishment of guidelines, reviewed by the whole community, we have the opportunity to include that expertise. The AIAA SBOS COS and its task groups are available to support that effort. 
December 21, 1992

SBOS-COS Sensor Systems Subcommittee

IR Sensor Calibration Standards Task Group

David Box

Ball Aerospace Corp.

Jerry Bronson

Boeing Company

Carol J. Bruegge

Jet Propulsion Laboratory

Raju Datla

NIST/Radiometric Physics Division

Roy Gavin

ITT Aerospace/Communications

Edward Koenig

8825 Beacon Woods Place

Ft. Wayne, IN 46804

Andrew Lazarewicz

Mission Research Corp

Paul Menzel

NOAA/NESDIS, E/RA 24

James M. Palmer

U of Arizona- Optical Sciences Center

David B. Pollock

Teledyne Brown Engineering

Richard Raper

Calspan Corp. MS 640

Arnold Air Force Base, TN 37389
Milton Triplett

Nichols Research Corp

Tom Wallace

Nichols Research Corporation

Mike Weinreb

NOAA/NESDIS, Code E/RA 14

Clair Wyat!

Space Dynamics Laboratory

Utah State University

Ray Young

Calspan Corp. MS 640

Arnold Air Force Base, TN 37389 


\section{APPENDIX 2}

\section{GUIDELINES FOR EVALUATING AND EXPRESSING THE UNCERTAINTY OF NIST MEASUREMENT RESULTS NIST TECHNICAL NOTE 1297}





\section{NIST Technical Note 1297 \\ 1994 Edition}

\section{Guidelines for Evaluating and Expressing the Uncertainty of NIST Measurement Results}

Barry N. Taylor and Chris E. Kuyatt

Physics Laboratory

National Institute of Standards and Technology

Gaithersburg, MD 20899

(Supersedes NIST Technical Note 1297, January 1993)

September 1994

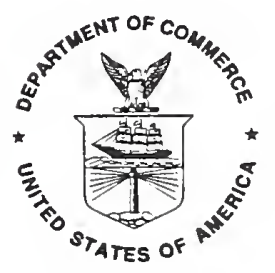

U.S. Department of Commerce

Ronald H. Brown, Secretary

Technology Administration

Mary L. Good, Under Secretary for Technology

National Institute of Standards and Technology

Arati Prabhakar, Director 



\section{Preface to the 1994 Edition}

The previous edition, which was the first, of this National Institute of Standards and Technology (NIST) Technical Note (TN 1297) was initially published in January 1993. A second printing followed shortly thereafter, and in total some 10000 copies were distributed to individuals at NIST and in both the United States at large and abroad - to metrologists, scientists, engineers, statisticians, and others who are concerned with measurement and the evaluation and expression of the uncertainty of the result of a measurement. On the whole, these individuals gave TN 1297 a very positive reception. We were, of course, pleased that a document intended as a guide to NIST staff was also considered to be of significant value to the international measurement community.

Several of the recipients of the 1993 edition of TN 1297 asked us questions concerning some of the points it addressed and some it did not. In view of the nature of the subject of evaluating and expressing measurement uncertainty and the fact that the principles presented in TN 1297 are intended to be applicable to a broad range of measurements, such questions were not at all unexpected.

It soon occurred to us that it might be helpful to the current and future users of TN 1297 if the most important of these questions were addressed in a new edition. To this end, we have added to the 1993 edition of TN 1297 a new appendix
- Appendix D-which attempts to clarify and give additional guidance on a number of topics, including the use of certain terms such as accuracy and precision. We hope that this new appendix will make this 1994 edition of TN 1297 even more useful than its predecessor.

We also took the opportunity provided us by the preparation of a new edition of TN 1297 to make very minor word changes in a few portions of the texr. These changes were made in order to recognize the official publication in October 1993 of the ISO Guide to the Expression of Uncertainty in Measurement on which TN 1297 is based (for example, the reference to the Guide was updated); and to bring TN 1297 into full harmony with the Guide (for example, "estimated correction" has been changed to simply "correction," and "can be asserted to lie" has been changed to "is believed to lie").

September 1994

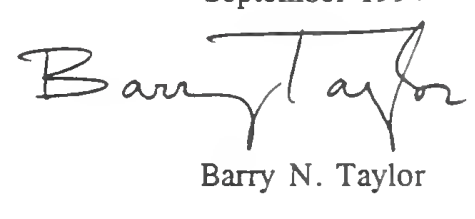

Ohaie \& Kuyat

Chris E. Kuyatt 



\section{FOREWORD}

(to the 1993 Edition)

Results of measurements and conclusions derived from them constitute much of the technical information produced by NIST. It is generally agreed that the usefulness of measurement results, and thus much of the information that we provide as an institution, is to a large extent determined by the quality of the statements of uncertainty that accompany them. For example, only if quantitative and thoroughly documented statements of uncertainty accompany the results of NIST calibrations can the users of our calibration services establish their level of traceability to the U.S. standards of measurement maintained at NIST.

Although the vast majority of NIST measurement results are accompanied by quantitative statements of uncertainty, there has never been a uniform approach at NIST to the expression of uncertainty. The use of a single approach within the Institute rather than many different approaches would ensure the consistency of our outputs, thereby simplifying their interpretation.

To address this issue, in July 1992 I appointed a NIST Ad Hoc Committee on Uncertainty Statements and charged it with recommending to me a NIST policy on this important topic. The members of the Committee were:

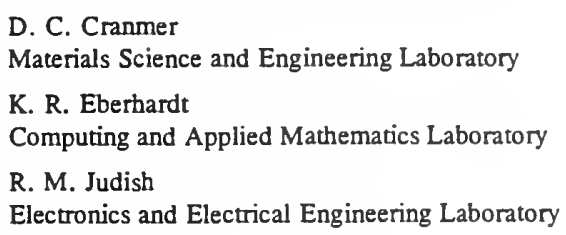

R. A. Kamper

Office of the Director, NIST/Boulder Laboratories

C. E. Kuyatt

Physics Laboratory

J. R. Rosenblatt

Computing and Applied Mathematics Laboratory

J. D. Simmons

Technology Services

L. E. Smith

Office of the Director, NIST; Chair

D. A. Swyt

Manufacturing Engineering Laboratory

B. N. Taylor

Physics Laboratory

R. L. Watters

Chemical Science and Technology Laboratory
This action was motivated in part by the emerging international consensus on the approach to expressing uncertainty in measurement recommended by the International Committee for Weights and Measures (CIPM). The movement toward the international adoption of the CIPM approach for expressing uncertainty is driven to a large extent by the global economy and marketplace; its worldwide use will allow measurements performed in different countries and in sectors as diverse as science, engineering, commerce, industry, and regulation to be more easily understood, interpreted, and compared.

At my request, the Ad Hoc Committee carefully reviewed the needs of NIST customers regarding statements of uncertainty and the compatibility of those needs with the CIPM approach. It concluded that the CIPM approach could be used to provide quantitative expressions of measurement uncertainty that would satisfy our customers' requirements. The Ad Hoc Committee then recommended to me a specific policy for the implementation of that approach at NIST. I enthusiastically accepted its recommendation and the policy has been incorporated in the NIST Administrative Manual. (It is also included in this Technical Note as Appendix C.)

To assist the NIST staff in putting the policy into practice, two members of the Ad Hoc Committee prepared this Technical Note. I believe that it provides a helpful discussion of the CIPM approach and, with its aid, that the NIST policy can be implemented without excessive difficulty. Further, I believe that because NIST statements of uncertainty resulting from the policy will be uniform among themselves and consistent with current international practice, the policy will help our customers increase their competitiveness in the national and international marketplaces.

January 1993

John W. Lyons

Director,

National Institute of Standards and Technology 



\section{GUIDELINES FOR EVALUATING AND EXPRESSING THE UNCERTAINTY OF NIST MEASUREMENT RESULTS}

\section{Introduction}

1.1 In October 1992, a new policy on expressing measurement uncertainty was instituted at NIST. This policy is set forth in "Statements of Uncertainty Associated With Measurement Results," Appendix E, NIST Technical Communications Program, Subchapter 4.09 of the Administrative Manual (reproduced as Appendix C of these Guidelines).

1.2 The new NIST policy is based on the approach to expressing uncertainty in measurement recommended by the $\mathrm{CIPM}^{1}$ in 1981 [1] and the elaboration of that approach given in the Guide to the Expression of Uncertainty in Measurement (hereafter called the Guide), which was prepared by individuals nominated by the BIPM, IEC, ISO, or OIML [2]. ${ }^{1}$ The CIPM approach is founded on Recommendation INC-1 (1980) of the Working Group on the Statement of Uncertainties [3]. This group was convened in 1980 by the BIPM as a consequence of a $1977^{2}$ request by the CIPM that the BIPM study the question of reaching an international consensus on expressing uncertainty in measurement. The request was initiated by then CIPM member and NBS Director E. Ambler. A $1985^{2}$ request by the CIPM to ISO asking it to develop a broadly applicable guidance document based on Recommendation INC-1 (1980) led to the development of the Guide. It is at present the most complete reference on the general application of the CIPM approach to expressing measurement uncertainty, and its development is giving further impetus to the worldwide adoption of that approach.

1.3 Although the Guide represents the current international view of how to express uncertainty in measurement based on the CIPM approach, it is a rather lengthy document. We have therefore prepared this Technical Note with the goal of succinctly presenting, in the context of the new NIST policy, those aspects of the Guide that will be of most use to the NIST staff in implementing that policy. We have also included some

'CIPM: Intemational Committee for Weights and Measures; BIPM: International Bureau of Weights and Measures; IEC: Intemational Electrotechnical Commission; ISO: International Organization for Standardization; OIML: International Organization of Legal Metrology.

${ }^{2}$ These dates have been corrected from those in the first (1993) edition of TN 1297 and in the Guide. suggestions that are not contained in the Guide or policy but which we believe are useful. However, none of the guidance given in this Technical Note is to be interpreted as NIST policy unless it is directly quoted from the policy itself. Such cases will be clearly indicated in the text.

1.4 The guidance given in this Technical Note is intended to be applicable to most, if not all, NIST measurement results, including results associated with

- international comparisons of measurement standards,

- basic research,

- applied research and engineering,

- calibrating client measurement standards,

- certifying standard reference materials, and

- generating standard reference data.

Since the Guide itself is intended to be applicable to similar kinds of measurement results, it may be consulted for additional details. Classic expositions of the statistical evaluation of measurement processes are given in references [4-7].

\section{Classification of Components of Uncertainty}

2.1 In general, the result of a measurement is only an approximation or estimate of the value of the specific quantity subject to measurement, that is, the measurand, and thus the result is complete only when accompanied by a quantitative statement of its uncertainty.

2.2 The uncerainty of the result of a measurement generally consists of several components which, in the CIPM approach, may be grouped into two categories according to the method used to estimate their numerical values:

A. those which are evaluated by statistical methods,

B. those which are evaluared by other means.

2.3 There is not always a simple correspondence between the classification of uncertainty components into categories $A$ and $B$ and the commonly used classification of uncertainty components as "random" and "systematic." The nature of an uncertainty component is conditioned by the use made of the corresponding quantity, that is, on how that 
quantity appears in the mathematical model that describes the measurement process. When the corresponding quantity is used in a different way, a "random" component may become a "systematic" component and vice versa. Thus the terms "random uncertainty" and "systematic uncertainty" can be misleading when generally applied. An alternative nomenclature that might be used is

"component of uncertainty arising from a random effect,"

"component of uncertainty arising from a systematic effect,"

where a random effect is one that gives rise to a possible random error in the current measurement process and a systematic effect is one that gives rise to a possible systematic error in the current measurement process. In principle, an uncertainty component arising from a systematic effect may in some cases be evaluated by method A while in other cases by method B (see subsection 2.2), as may be an uncertainty component arising from a random effect.

NOTE - The difference between error and uncertainty should always be borne in mind. For example, the result of a measurement after correction (see subsection 5.2) can unknowably be very close to the unknown value of the measurand, and thus have negligible error, even though it may have a large uncertainty (see the Guide [2]).

2.4 Basic to the CIPM approach is representing each component of uncertainty that contributes to the uncertainty of a measurement result by an estimated standard deviation, termed standard uncertainty with suggested symbol $u_{i}$, and equal to the positive square root of the estimated variance $u_{i}^{2}$.

2.5 It follows from subsections 2.2 and 2.4 that an uncertainty component in category $\mathrm{A}$ is represented by a statistically estimated standard deviation $s_{i}$, equal to the positive square root of the statistically estimated variance $s_{i}^{2}$, and the associated number of degrees of freedom $v_{i}$. For such a component the standard uncertainty is $u_{i}=s_{i}$.

The evaluation of uncertainty by the statistical analysis of series of observations is termed a Type A evaluation (of uncertainty).

2.6 In a similar manner, an uncertainty component in category $\mathrm{B}$ is represented by a quantity $u_{j}$, which may be considered an approximation to the corresponding standard deviation; it is equal to the positive square root of $u_{j}^{2}$, which may be considered an approximation to the corresponding variance and which is obtained from an assumed probability distribution based on all the available information (see section 4). Since the quantity $u_{j}^{2}$ is treated like a variance and $u_{j}$ like a standard deviation, for such a component the standard uncertainty is simply $u_{j}$.

The evaluation of uncertainty by means other than the statistical analysis of series of observations is termed a Type B evaluation (of uncertainty).

2.7 Correlations between components (of either category) are characterized by estimated covariances [see Appendix A, Eq. (A-3)] or estimated correlation coefficients.

\section{Type A Evaluation of Standard Uncertainty}

A Type A evaluation of standard uncertainty may be based on any valid statistical method for treating data. Examples are calculating the standard deviation of the mean of a series of independent observations [see Appendix A, Eq. (A-5)]; using the method of least squares to fit a curve to data in order to estimate the parameters of the curve and their standard deviations; and carrying out an analysis of variance (ANOVA) in order to identify and quantify random effects in certain kinds of measurements. If the measurement situarion is especially complicated, one should consider obtaining the guidance of a staristician. The NIST staff can consult and collaborate in the development of statistical experiment designs, analysis of data, and other aspects of the evaluation of measurements with the Statistical Engineering Division, Computing and Applied Mathematics Laboratory. Inasmuch as this Technical Note does not attempt to give detailed statistical techniques for carrying out Type A evaluations, references [4-7], and reference [8] in which a general approach to quality control of measurement systems is set forth, should be consulted for basic principles and additional references.

\section{Type B Evaluation of Standard Uncertainty}

4.1 A Type B evaluarion of standard uncertainty is usually based on scientific judgment using all the relevant information available, which may include

- previous measurement data,

- experience with, or general knowledge of, the behavior and property of relevant materials and instruments,

- manufacturer's specifications,

- data provided in calibration and other reports, and

- uncertainties assigned to reference data taken from handbooks. 
Some examples of Type B evaluations are given in subsections 4.2 to 4.6 .

4.2 Convert a quoted uncertainty that is a stated multiple of an estimated standard deviation to a standard uncertainty by dividing the quoted uncertainty by the multiplier.

4.3 Convert a quoted uncertainty that defines a "confidence interval" having a stated level of confidence (see subsection 5.5), such as 95 or 99 percent, to a standard uncertainty by treating the quoted uncertainty as if a normal distribution had been used to calculate it (unless otherwise indicated) and dividing it by the appropriate factor for such a distribution. These factors are 1.960 and 2.576 for the two level's of confidence given (see also the last line of Table B.1 of Appendix B).

4.4 Model the quantity in question by a normal distribution and estimate lower and upper limits $a_{-}$and $a_{+}$ such that the best estimated value of the quantity is $\left(a_{+}+a_{-}\right) / 2$ (i.e., the center of the limits) and there is 1 chance out of 2 (i.e., a 50 percent probability) that the value of the quantity lies in the interval $a_{-}$to $a_{+}$. Then $u_{j} \approx 1.48 a$, where $a=\left(a_{+}-a_{-}\right) / 2$ is the half-width of the interval.

4.5 Model the quantity in question by a normal distribution and estimate lower and upper limits $a_{-}$and $a_{+}$ such that the best estimated value of the quantity is $\left(a_{+}+a_{-}\right) / 2$ and there is about a 2 out of 3 chance (i.e., a 67 percent probability) that the value of the quantity lies in the interval $a_{-}$to $a_{+}$. Then $u_{j} \approx a$, where $a=\left(a_{+}-a_{-}\right) / 2$.

4.6 Estimate lower and upper limits $a_{-}$and $a_{+}$for the value of the quantity in question such that the probability that the value lies in the interval $a_{-}$to $a_{+}$is, for all practical purposes, 100 percent. Provided that there is no contradictory information, treat the quantity as if it is equally probable for its value to lie anywhere within the interval $a_{-}$to $a_{+}$; that is, model it by a uniform or rectangular probability distribution. The best estimate of the value of the quantity is then $\left(a_{+}+a_{-}\right) / 2$ with $u_{j}=a / \sqrt{3}$, where $a=\left(a_{+}-a_{-}\right) / 2$.

If the distribution used to model the quantity is triangular rather than rectangular, then $u_{j}=a / \sqrt{6}$.

If the quantity in question is modeled by a normal distribution as in subsections 4.4 and 4.5 , there are no finite limits that will contain 100 percent of its possible values. However, plus and minus 3 standard deviations about the mean of a normal distribution corresponds to
99.73 percent limits. Thus, if the limits $a_{-}$and $a_{+}$of a normally distributed quantity with mean $\left(a_{+}+a_{-}\right) / 2$ are considered to contain "almost all" of the possible values of the quantity, that is, approximately 99.73 percent of them, then $u_{j} \approx a / 3$, where $a=\left(a_{+}-a_{-}\right) / 2$.

The rectangular distribution is a reasonable default model in the absence of any other information. But if it is known that values of the quantity in question near the center of the limits are more likely than values close to the limits, a triangular or a normal distribution may be a better model.

4.7 Because the reliability of evaluations of components of uncertainty depends on the quality of the information available, it is recommended that all parameters upon which the measurand depends be varied to the fullest extent practicable so that the evaluations are based as much as possible on observed data. Whenever feasible, the use of empirical models of the measurement process founded on long-term quantitative data, and the use of check standards and control charts that can indicate if a measurement process is under statistical control, should be part of the effort to obtain reliable evaluations of components of uncertainty [8]. Type A evaluations of uncertainty based on limited data are not necessarily more reliable than soundly based Type B evaluations.

\section{Combined Standard Uncertainty}

5.1 The combined standard uncertainty of a measurement result, suggested symbol $u_{\mathrm{c}}$, is taken to represent the estimated standard deviation of the result. It is obtained by combining the individual standard uncertainties $u_{i}$ (and covariances as appropriate), whether arising from a Type $\mathrm{A}$ evaluation or a Type B evaluation, using the usual method for combining standard deviations. This method, which is summarized in Appendix A [Eq. (A-3)], is often called the law of propagation of uncertainty and in common parlance the "root-sum-of-squares" (square root of the sum-of-thesquares) or "RSS" method of combining uncertainty components estimated as standard deviations.

NOTE - The NIST policy also allows the use of established and documented methods equivalent to the "RSS" method, such as the numerically based "bootstrap" (see Appendix C).

5.2 It is assumed that a correction (or correction factor) is applied to compensate for each recognized systematic effect that significantly influences the measurement result and that every effort has been made to identify such effects. The relevant uncertainty to associate with each recognized systematic effect is then the standard uncertainty of the 
applied correction. The correction may be either positive, negative, or zero, and its standard uncertainty may in some cases be obtained from a Type A evaluation while in other cases by a Type B evaluation.

\begin{abstract}
NOTES
1 The uncertainty of a correction applied to a measurement result to compensate for a systematic effect is not the systematic error in the measurement result due to the effect. Rather, it is a measure of the uncertainty of the result due to incomplete knowledge of the required value of the correction. The terms "error" and "uncertainty" should not be confused (see also the note of subsection 2.3).

2 Although it is strongly recommended that corrections be applied for all recognized significant systematic effects, in some cases it may not be practical because of limited resources. Nevertheless, the expression of uncertainty in such cases should conform with these guidelines to the fullest possible extent (see the Guide [2]).
\end{abstract}

5.3 The combined standard uncertainty $u_{\mathrm{c}}$ is a widely employed measure of uncertainty. The NIST policy on expressing uncertainty states that (see Appendix C):

Commonly, $u_{c}$ is used for reporting results of determinations of fundamental constants, fundamental metrological research, and international comparisons of realizations of SI units.

Expressing the uncertainty of NIST's primary cesium frequency standard as an estimated standard deviation is an example of the use of $u_{\mathrm{c}}$ in fundamental metrological research. It should also be noted that in a 1986 recommendation [9], the CIPM requested that what is now termed combined standard uncertainty $u_{c}$ be used "by all participants in giving the results of all international comparisons or other work done under the auspices of the CIPM and Comités Consultatifs."

5.4 In many practical measurement situations, the probability distribution characterized by the measurement result $y$ and its combined standard uncertainty $u_{c}(y)$ is approximately normal (Gaussian). When this is the case and $u_{c}(y)$ itself has negligible uncertainty (see Appendix B), $u_{\mathrm{c}}(y)$ defines an interval $y-u_{\mathrm{c}}(y)$ to $y+u_{\mathrm{c}}(y)$ about the measurement result $y$ within which the value of the measurand $Y$ estimated by $y$ is believed to lie with a level of confidence of approximately 68 percent. That is, it is believed with an approximate level of confidence of 68 percent that $y-u_{\mathrm{c}}(y) \leq Y \leq y+u_{\mathrm{c}}(y)$, which is commonly written as $Y=y \pm u_{c}(y)$.

The probability distribution characterized by the measurement result and its combined standard uncertainty is approximately normal when the conditions of the Central Limit Theorem are met. This is the case, often encountered in practice, when the estimate $y$ of the measurand $Y$ is not determined directly but is obtained from the estimated values of a significant number of other quantities [see Appendix A, Eq. (A-1)] describable by well-behaved probability distributions, such as the normal and rectangular distributions; the standard uncertainties of the estimates of these quantities contribute comparable amounts to the combined standard uncertainty $u_{\mathrm{c}}(y)$ of the measurement result $y$; and the linear approximation implied by Eq. (A-3) in Appendix $\mathrm{A}$ is adequate.

NOTE - If $u_{c}(y)$ has non-negligible uncertainty, the level of confidence will differ from 68 percent. The procedure given in Appendix B has been proposed as a simple expedient for approximating the level of confidence in these cases.

5.5 The term "confidence interval" has a specific definition in statistics and is only applicable to intervals based on $u_{\mathrm{c}}$ when certain conditions are met, including that all components of uncertainty that contribute to $u_{c}$ be obtained from Type A evaluations. Thus, in these guidelines, an interval based on $u_{c}$ is viewed as encompassing a fraction $p$ of the probability distribution characterized by the measurement result and its combined standard uncertainty, and $p$ is the coverage probability or level of confidence of the interval.

\section{Expanded Uncertainty}

6.1 Although the combined standard uncertainty $u_{\mathrm{c}}$ is used to express the uncertainty of many NIST measurement results, for some commercial, industrial, and regulatory applications of NIST results (e.g., when health and safety are concerned), what is often required is a measure of uncertainty that defines an interval about the measurement result $y$ within which the value of the measurand $Y$ is confidently believed to lie. The measure of uncertainty intended to meet this requirement is termed expanded uncertainty, suggested symbol $U$, and is obtained by multiplying $u_{c}(y)$ by a coverage factor, suggested symbol $k$. Thus $U=k u_{\mathrm{c}}(y)$ and it is confidently believed that $y-U \leq Y \leq y+U$, which is commonly written as $Y=y \pm U$.

It is to be understood that subsection 5.5 also applies to the interval defined by expanded uncertainty $U$.

6.2 In general, the value of the coverage factor $k$ is chosen on the basis of the desired level of confidence to be associated with the interval defined by $U=k u_{\mathrm{c}}$. Typically, $k$ is in the range 2 to 3 . When the normal distribution applies and $u_{\mathrm{c}}$ has negligible uncertainty (see subsection 5.4), $U=2 u_{\mathrm{c}}$ (i.e., $k=2$ ) defines an interval having a 
level of confidence of approximately 95 percent, and $U=3 u_{c}$ (i.e., $k=3$ ) defines án interval having a level of confidence greater than 99 percent.

NOTE - For a quantity $z$ described by a normal distribution with expectation $\mu_{2}$ and standard deviation $\sigma$, the interval $\mu_{2} \pm k \sigma$ encompasses $68.27,90,95.45,99$, and 99.73 percent of the distribution for $k=1, k=1.645, k=2, k=2.576$, and $k=3$, respectively (see the last line of Table B.1 of Appendix B).

6.3 Ideally, one would like to be able to choose a specific value of $k$ that produces an interval corresponding to a welldefined level of confidence $p$, such as 95 or 99 percent; equivalently, for a given value of $k$, one would like to be able to state unequivocally the level of confidence associated with that interval. This is difficult to do in practice because it requires knowing in considerable detail the probability distribution of each quantity upon which the measurand depends and combining those distributions to obtain the distribution of the measurand.

NOTE - The more thorough the investigation of the possible existence of non-trivial systematic effects and the more complete the data upon which the estimates of the corrections for such effects are based, the closer one can get to this ideal (see subsections 4.7 and 5.2).

6.4 The CIPM approach does not specify how the relation between $k$ and $p$ is to be established. The Guide [2] and Dietrich [10] give an approximate solution to this problem (see Appendix B); it is possible to implement others which also approximate the result of combining the probability distributions assumed for each quantity upon which the measurand depends, for example, solutions based on numerical methods.

6.5 In light of the discussion of subsections 6.1-6.4, and in keeping with the practice adopted by other national standards laboratories and several metrological organizations, the stated NIST policy is (see Appendix C):

Use expanded uncertainty $U$ to report the results of all NIST measurements other than those for which $u_{c}$ has traditionally been employed. To be consistent with current international practice, the value of $k$ to be used at NIST for calculating $U$ is, by convention, $k=2$. Values of $k$ other than 2 are only to be used for specific applications dictated by established and documented requirements.

An example of the use of a value of $k$ other than 2 is taking $k$ equal to a $t$-factor obtained from the $t$-distribution when $u_{\mathrm{c}}$ has low degrees of freedom in order to meet the dictated requirement of providing a value of $U=k u_{c}$ that defines an interval having a level of confidence close to 95 percent.
(See Appendix B for a discussion of how a value of $k$ that produces such a value of $U$ might be approximated.)

6.6 The NIST policy provides for exceptions as follows (see Appendix C):

It is understood that any valid statistical method that is technically justified under the existing circumstances may be used to determine the equivalent of $u_{i}, u_{c}$, or $U$. Further, it is recognized that international, national, or contractual agreements to which NIST is a party may occasionally require deviation from NIST policy. In both cases, the report of uncertainty must document what was done and why.

\section{Reporting Uncertainty}

7.1 The stated NIST policy regarding reporting uncertainty is (see Appendix C):

Report $U$ together with the coverage factor $k$ used to obtain it, or report $u_{\mathrm{c}}$.

When reporting a measurement result and its uncertainty, include the following information in the report itself or by referring to a published document:

- A list of all components of standard uncertainty, together with their degrees of freedom where appropriate, and the resulting value of $u_{\mathrm{c}}$. The components should be identified according to the method used to estimate their numerical values:

A. those which are evaluated by statistical methods,

B. those which are evaluated by other means.

- A detailed description of how each component of standard uncertainty was evaluated.

- A description of how $k$ was chosen when $k$ is not taken equal to 2 .

It is often desirable to provide a probability interpretation, such as a level of confidence, for the interval defined by $U$ or $u_{\mathrm{c}}$. When this is done, the basis for such a statement must be given.

7.2 The NIST requirement that a full description of what was done be given is in keeping with the generally accepted view that when reporting a measurement result and its uncertainty, it is preferable to err on the side of providing 
too much information rather than too little. However, when such details are provided to the úsers of NIST measurement results by referring to published documents, which is often the case when such results are given in calibration and test reports and certificates, it is imperative that the referenced documents be kept up-to-date so that they are consistent with the measurement process in current use.

7.3 The last paragraph of the NIST policy on reporting uncertainty (see subsection 7.1 above) refers to the desirability of providing a probability interpretation, such as a level of confidence, for the interval defined by $U$ or $u_{c}$. The following examples show how this might be done when the numerical result of a measurement and its assigned uncertainty is reported, assuming that the published detailed description of the measurement provides a sound basis for the statements made. (In each of the three cases, the quantity whose value is being reported is assumed to be a nominal $100 \mathrm{~g}$ standard of mass $m_{\mathrm{s}}$.)

$m_{\mathrm{s}}=(100.02147 \pm 0.00070) \mathrm{g}$, where the number following the symbol \pm is the numerical value of an expanded uncertainty $U=k u_{\mathrm{c}}$, with $U$ determined from a combined standard uncertainty (i.e., estimated standard deviation) $u_{\mathrm{c}}=0.35 \mathrm{mg}$ and a coverage factor $k=2$. Since it can be assumed that the possible estimated values of the standard are approximately normally distributed with approximate standard deviation $u_{c}$, the unknown value of the standard is believed to lie in the interval defined by $U$ with a level of confidence of approximately 95 percent.

$m_{\mathrm{s}}=(100.02147 \pm 0.00079) \mathrm{g}$, where the number following the symbol \pm is the numerical value of an expanded uncertainty $U=k u_{c}$, with $U$ determined from a combined standard uncertainty (i.e., estimated standard deviation) $u_{\mathrm{c}}=0.35 \mathrm{mg}$ and a coverage factor $k=2.26$ based on the $t$-distribution for $v=9$ degrees of freedom, and defines an interval within which the unknown value of the standard is believed to lie with a level of confidence of approximately 95 percent.

$m_{\mathrm{s}}=100.02147 \mathrm{~g}$ with a combined standard uncertainty (i.e., estimated standard deviation) of $u_{c}=0.35 \mathrm{mg}$. Since it can be assumed that the possible estimated values of the standard are approximately normally distributed with approximate standard deviation $u_{c}$, the unknown value of the standard is believed to lie in the interval $m_{\mathrm{s}} \pm u_{\mathrm{c}}$ with a level of confidence of approximately 68 percent.

When providing such probability interpretations of the intervals defined by $U$ and $u_{c}$, subsection 5.5 should be recalled. In this regard, the interval defined by $U$ in the second example might be a conventional confidence interval (at least approximately) if all the components of uncertainty are obtained from Type A evaluations.

7.4 Some users of NIST measurement results may automatically interpret $U=2 u_{\mathrm{c}}$ and $u_{\mathrm{c}}$ as quantities that define intervals having levels of confidence corresponding to those of a normal distribution, namely, 95 percent and 68 percent, respectively. Thus, when reporting either $U=2 u_{\mathrm{c}}$ or $u_{\mathrm{c}}$, if it is known that the interval which $U=2 u_{\mathrm{c}}$ or $u_{\mathrm{c}}$ defines has a level of confidence that differs significantly from 95 percent or 68 percent, it should be so stated as an aid to the users of the measurement result. In keeping with the NIST policy quoted in subsection 6.5 , when the measure of uncertainty is expanded uncertainty $U$, one may use a value of $k$ that does lead to a value of $U$ that defines an interval having a level of confidence of 95 percent if such a value of $U$ is necessary for a specific application dictated by an established and documented requirement.

7.5 In general, it is not possible to know in detail all of the uses to which a particular NIST measurement result will be put. Thus, it is usually inappropriate to include in the uncertainty reported for a NIST result any component that arises from a NIST assessment of how the result might be employed; the quoted uncertainty should normally be the actual uncertainty obtained at NIST.

7.6 It follows from subsection 7.5 that for standards sent by customers to NIST for calibration, the quoted uncertainty should not normally include estimates of the uncertainties that may be introduced by the return of the standard to the customer's laboratory or by its use there as a reference standard for other measurements. Such uncertainties are due, for example, to effects arising from transportation of the standard to the customer's laboratory, including mechanical damage; the passage of time; and differences between the environmental conditions at the customer's laboratory and at NIST. A caution may be added to the reported uncertainty if any such effects are likely to be significant and an additional uncertainty for them may be estimated and quoted. If, for the convenience of the customer, this additional uncertainty is combined with the uncertainty obtained at NIST, a clear statement should be included explaining that this has been done.

Such considerations are also relevant to the uncertainties assigned to certified devices and materials sold by NIST. However, well-justified, normal NIST practices, such as including a component of uncertainty to account for the 
instability of the device or material when it is known to be significant, are clearly necessary if the assigned uncertainties are to be meaningful.

\section{References}

[1] CIPM, BIPM Proc.-Verb. Com. Int. Poids et Mesures 49, 8-9, 26 (1981) (in French); P. Giacomo, "News from the BIPM," Metrologia 18, 41-44 (1982).

[2] ISO, Guide to the Expression of Uncertainty in Measurement (International Organization for Standardization, Geneva, Switzerland, 1993). This Guide was prepared by ISO Technical Advisory Group 4 (TAG 4), Working Group 3 (WG 3). ISO/TAG 4 has as its sponsors the BIPM, IEC, IFCC (International Federation of Clinical Chemistry), ISO, IUPAC (International Union of Pure and Applied Chemistry), IUPAP (International Union of Pure and Applied Physics), and OIML. Although the individual members of WG 3 were nominated by the BIPM, IEC, ISO, or OIML, the Guide is published by ISO in the name of all seven organizations. NIST staff members may obtain a single copy of the Guide from the NIST Calibration Program.

[3] R. Kaarls, "Rapport du Groupe de Travail sur l'Expression des Incertitudes au Comité International des Poids et Mesures," Proc.-Verb. Com. Int. Poids et Mesures 49, A1-A12 (1981) (in French); P. Giacomo, "News from the BIPM," Metrologia 17, 69-74 (1981). (Note that the final English-language version of Recommendation INC-1 (1980), published in an internal BIPM report, differs slightly from that given in the latter reference but is consistent with the authoritative French-language version given in the former reference.)

[4] C. Eisenhart, "Realistic Evaluation of the Precision and Accuracy of Instrument Calibration Systems," J. Res. Natl. Bur. Stand. (U.S.) 67C, 161-187 (1963). Reprinted, with corrections, in Precision Measurement and Calibration: Statistical Concepts and Procedures, NBS Special Publication 300, Vol. I, H. H. Ku, Editor (U.S. Government Printing Office, Washington, DC, 1969), pp. 21-48.

[5] J. Mandel, The Statistical Analysis of Experimental Data (Interscience-Wiley Publishers, New York, NY, 1964, out of print; corrected and reprinted, Dover Publishers, New York, NY, 1984).
[6] M. G. Natrella, Experimental Statistics, NBS Handbook 91 (U.S. Government Printing Office, Washington, DC, 1963; reprinted October 1966 with corrections).

[7] G. E. P. Box, W. G. Hunter, and J. S. Hunter, Statistics for Experimenters (John Wiley \& Sons, New York, NY, 1978).

[8] C. Croarkin, Measurement Assurance Programs, Part II: Development and Implementation, NBS Special Publication 676-II (U.S. Government Printing Office, Washington, DC, 1985).

[9] CIPM, BIPM Proc.-Verb. Com. Int. Poids et Mesures 54, 14, 35 (1986) (in French); P. Giacomo, "News from the BIPM," Metrologia 24, 45-51 (1987).

[10] C. F. Dietrich, Uncertainty, Calibration and Probability, second edition (Adam Hilger, Bristol, U.K., 1991), chapter 7.

\section{Appendix A}

Law of Propagation of Uncertainty

A.1 In many cases a measurand $Y$ is not measured directly, but is determined from $N$ other quantities $X_{1}, X_{2}, \ldots, X_{N}$ through a functional relation $f$ :

$$
Y=f\left(X_{1}, X_{2}, \ldots, X_{N}\right) .
$$

Included among the quantities $X_{i}$ are corrections (or correction factors) as described in subsection 5.2, as well as quantities that take into account other sources of variability, such as different observers, instruments, samples, laboratories, and times at which observations are made (e.g., different days). Thus the function $f$ of Eq. (A-1) should express not simply a physical law but a measurement process, and in particular, it should contain all quantities that can contribute a significant uncertainty to the measurement result.

A.2 An estimate of the measurand or output quantity $Y$, denoted by $y$, is obtained from Eq. (A-1) using input estimates $x_{1}, x_{2}, \ldots, x_{N}$ for the values of the $N$ input quantities $X_{1}, X_{2}, \ldots, X_{N}$. Thus the output estimate $y$, which is the result of the measurement, is given by

$$
y=f\left(x_{1}, x_{2}, \ldots, x_{N}\right) .
$$


A.3 The combined standard uncertainty of the measurement result $y$, designated by $u_{c}(y)$ and taken to represent the estimated standard deviation of the result, is the positive square root of the estimated variance $u_{c}^{2}(y)$ obtained from

$$
\begin{aligned}
u_{\mathrm{c}}^{2}(y)= & \sum_{i=1}^{N}\left[\frac{\partial f}{\partial x_{i}}\right]^{2} u^{2}\left(x_{i}\right) \\
& +2 \sum_{i=1}^{N-1} \sum_{j=i+1}^{N} \frac{\partial f}{\partial x_{i}} \frac{\partial f}{\partial x_{j}} u\left(x_{i}, x_{j}\right) .
\end{aligned}
$$

Equation (A-3) is based on a first-order Taylor series approximation of $Y=f\left(X_{1}, X_{2}, \ldots, X_{N}\right)$ and is conveniently referred to as the law of propagation of uncertainty. The partial derivatives $\partial f / \partial x_{i}$ (often referred to as sensitivity coefficients) are equal to $\partial f / \partial X_{i}$ evaluated at $X_{i}=x_{i} ; u\left(x_{i}\right)$ is the standard uncertainty associated with the input estimate $x_{i}$; and $u\left(x_{i}, x_{j}\right)$ is the estimated covariance associated with $x_{i}$ and $x_{j}$.

A.4 As an example of a Type A evaluation, consider an input quantity $X_{i}$ whose value is estimated from $n$ independent observations $X_{i, k}$ of $X_{i}$ obtained under the same conditions of measurement. In this case the input estimate $x_{i}$ is usually the sample mean

$$
x_{i}=\bar{X}_{i}=\frac{1}{n} \sum_{k=1}^{n} X_{i, k},
$$

and the standard uncertainty $u\left(x_{i}\right)$ to be associated with $x_{i}$ is the estimated standard deviation of the mean

$$
\begin{aligned}
u\left(x_{i}\right) & =s\left(\bar{X}_{i}\right) \\
& =\left[\frac{1}{n(n-1)} \sum_{k=1}^{n}\left(X_{i, k}-\bar{X}_{i}\right)^{2}\right]^{1 / 2} .
\end{aligned}
$$

A.5 As an example of a Type B evaluation, consider an input quantity $X_{i}$ whose value is estimated from an assumed rectangular probability distribution of lower limit $a_{-}$and upper limit $a_{+}$. In this case the input estimate is usually the expectation of the distribution

$$
x_{i}=\left(a_{+}+a_{-}\right) / 2 \text {, }
$$

and the standard uncertainty $u\left(x_{i}\right)$ to be associated with $x_{i}$ is the positive square root of the variance of the distribution

$$
u\left(x_{i}\right)=a / \sqrt{3},
$$

where $a=\left(a_{+}-a_{-}\right) / 2$ (see subsection 4.6).

NOTE - When $x_{i}$ is obcained from an assumed distribution, the associated variance is appropriately written as $u^{2}\left(X_{i}\right)$ and the associated standard uncertainty as $u\left(X_{i}\right)$, but for simpliciry, $u^{2}\left(x_{i}\right)$ and $u\left(x_{i}\right)$ are used. Similar considerations apply to the symbols $u_{c}^{2}(y)$ and $u_{c}(y)$.

\section{Appendix B}

\section{Coverage Factors}

B.1 This appendix summarizes a conventional procedure, given by the Guide [2] and Dietrich [10], intended for use in calculating a coverage factor $k$ when the conditions of the Central Limit Theorem are met (see subsection 5.4) and (1) a value other than $k=2$ is required for a specific application dictated by an established and documented requirement; and (2) that value of $k$ must provide an interval having a level of confidence close to a specified value. More specifically, it is intended to yield a coverage factor $k_{p}$ that produces an expanded uncertainty $U_{p}=k_{p} u_{c}(y)$ that defines an interval $y-U_{p} \leq Y \leq$ $y+U_{p}$, which is commonly written as $Y=y \pm U_{p}$, having an approximate level of confidence $p$.

The four-step procedure is included in these guidelines because it is expected to find broad acceptance internationally, due in part to its computational convenience, in much the same way that $k=2$ has become the conventional coverage factor. However, although the procedure is based on a proven approximation, it should not be interpreted as being rigourous because the approximation is extrapolated to situations where its applicability has yet to be fully investigated.

B.2 To estimate the value of such a coverage factor requires taking into account the uncertainty of $u_{c}(y)$, that is, how well $u_{\mathrm{c}}(y)$ estimates the standard deviation associated with the measurement result. For an estimate of the standard deviation of a normal distribution, the degrees of freedom of the estimate, which depends on the size of the sample on which the estimate is based, is a measure of its uncertainty. For a combined standard uncertainty $u_{\mathrm{c}}(y)$, the "effective degrees of freedom" $v_{\text {eff }}$ of $u_{\mathrm{c}}(y)$, which is approximated by appropriately combining the degrees of freedom of its components, is a measure of its uncertainty. Hence $v_{\text {eff }}$ is a key factor in determining $k_{p}$. For example, if $v_{\text {eff }}$ is less than about 11 , simply assuming that the uncertainty of $u_{\mathrm{c}}(y)$ is negligible and taking $k=2$ may be inadequate if an expanded uncertainty $U=k u_{c}(y)$ that defines an interval having a level of confidence close to 95 percent is required for a specific application. More 
specifically, according to Table B.1 (to be discussed below), if $v_{\text {eff }}=8, k_{95}=2.3$ rather than 2.0. In this case, and in other similar cases where $v_{\text {eff }}$ of $u_{c}(y)$ is comparatively small and an interval having a level of confidence close to a specified level is required, it is unlikely that the uncertainty of $u_{\mathrm{c}}(y)$ would be considered negligible. Instead, the small value of $v_{\text {eff }}$, and thus the uncertainty of $u_{\mathrm{c}}(y)$, would probably be taken into account when determining $k_{p}$.

B.3 The four-step procedure for calculating $k_{p}$ is as follows:

1) Obtain $y$ and $u_{c}(y)$ as indicated in Appendix A.

2) Estimate the effective degrees of freedom $v_{\text {eff }}$ of $u_{c}(y)$ from the Welch-Satterthwaite formula

$$
v_{\mathrm{eff}}=\frac{u_{\mathrm{c}}^{4}(y)}{\sum_{i=1}^{N} \frac{c_{i}^{4} u^{4}\left(x_{i}\right)}{v_{i}}}
$$

where $c_{i} \equiv \partial f / \partial x_{i}$, all of the $u\left(x_{i}\right)$ are mutually statistically independent, $v_{i}$ is the degrees of freedom of $u\left(x_{i}\right)$, and

$$
v_{\text {eff }} \leq \sum_{i=1}^{N} v_{i} .
$$

The degrees of freedom of a standard uncertainty $u\left(x_{i}\right)$ obtained from a Type A evaluation is determined by appropriate statistical methods [7]. In the common case discussed in subsection A.4 where $x_{i}=\bar{X}_{i}$ and $u\left(x_{i}\right)=$ $s\left(\bar{X}_{i}\right)$, the degrees of freedom of $u\left(x_{i}\right)$ is $v_{i}=n-1$. If $m$ parameters are estimated by fitting a curve to $n$ data points by the method of least squares, the degrees of freedom of the standard uncertainty of each paramter is $n-m$.

The degrees of freedom to associate with a standard uncertainty $u\left(x_{i}\right)$ obtained from a Type B evaluation is more problematic. However, it is common practice to carry out such evaluations in a manner that ensures that an underestimation is avoided. For example, when lower and upper limits $a_{-}$and $a_{+}$are set as in the case discussed in subsection A.5, they are usually chosen in such a way that the probability of the quantity in question lying outside these limits is in fact extremely small. Under the assumption that this practice is followed, the degrees of freedom of $u\left(x_{i}\right)$ may be taken to be $v_{i} \rightarrow \infty$.

NOTE - See the Guide [2] for a possible way to estimate $\psi_{i}$ when this assumption is not justified.

3) Obtain the $t$-factor $t_{p}\left(v_{\text {eff }}\right)$ for the required level of confidence $p$ from a table of values of $t_{p}(v)$ from the $t$-distribution, such as Table B. 1 of this Appendix. If $v_{\text {eff }}$ is not an integer, which will usually be the case, either interpolate or truncate $v_{\text {eff }}$ to the next lower integer.

4) Take $k_{p}=t_{p}\left(v_{\text {eff }}\right)$ and calculate $U_{p}=k_{p} u_{c}(y)$. 
Table B.1 - Value of $t_{p}(v)$ from the $t$-distribution for degrees of freedom $v$ that defines an interval $-t_{p}(v)$ to $+t_{p}(v)$ that encompasses the fraction $p$ of the distribution

\begin{tabular}{|c|c|c|c|c|c|c|}
\hline \multirow{2}{*}{$\begin{array}{c}\text { Degrees of } \\
\text { freedom } \\
v\end{array}$} & \multicolumn{6}{|c|}{ Fraction $p$ in percent } \\
\hline & $68.27^{(\mathrm{a})}$ & 90 & 95 & $95.45^{(a)}$ & 99 & $99.73^{(a)}$ \\
\hline 1 & 1.84 & 6.31 & 12.71 & 13.97 & 63.66 & 235.80 \\
\hline 2 & 1.32 & 2.92 & 4.30 & 4.53 & 9.92 & 19.21 \\
\hline 3 & 1.20 & 2.35 & 3.18 & 3.31 & 5.84 & 9.22 \\
\hline 4 & 1.14 & 2.13 & 2.78 & 2.87 & 4.60 & 6.62 \\
\hline 5 & 1.11 & 2.02 & 2.57 & 2.65 & 4.03 & 5.51 \\
\hline 6 & 1.09 & 1.94 & 2.45 & 2.52 & 3.71 & 4.90 \\
\hline 7 & 1.08 & 1.89 & 2.36 & 2.43 & 3.50 & 4.53 \\
\hline 8 & 1.07 & 1.86 & 2.31 & 2.37 & 3.36 & 4.28 \\
\hline 9 & 1.06 & 1.83 & 2.26 & 2.32 & 3.25 & 4.09 \\
\hline 10 & 1.05 & 1.81 & 2.23 & 2.28 & 3.17 & 3.96 \\
\hline 11 & 1.05 & 1.80 & 2.20 & 2.25 & 3.11 & 3.85 \\
\hline 12 & 1.04 & 1.78 & 2.18 & 2.23 & 3.05 & 3.76 \\
\hline 13 & 1.04 & 1.77 & 2.16 & 2.21 & 3.01 & 3.69 \\
\hline 14 & 1.04 & 1.76 & 2.14 & 2.20 & 2.98 & 3.64 \\
\hline 15 & 1.03 & 1.75 & 2.13 & 2.18 & 2.95 & 3.59 \\
\hline 16 & 1.03 & 1.75 & 2.12 & 2.17 & 2.92 & 3.54 \\
\hline 17 & 1.03 & 1.74 & 2.11 & 2.16 & 2.90 & 3.51 \\
\hline 18 & 1.03 & 1.73 & 2.10 & 2.15 & 2.88 & 3.48 \\
\hline 19 & 1.03 & 1.73 & 2.09 & 2.14 & 2.86 & 3.45 \\
\hline 20 & 1.03 & 1.72 & 2.09 & 2.13 & 2.85 & 3.42 \\
\hline 25 & 1.02 & 1.71 & 2.06 & 2.11 & 2.79 & 3.33 \\
\hline 30 & 1.02 & 1.70 & 2.04 & 2.09 & 2.75 & 3.27 \\
\hline 35 & 1.01 & 1.70 & 2.03 & 2.07 & 2.72 & 3.23 \\
\hline 40 & 1.01 & 1.68 & 2.02 & 2.06 & 2.70 & 3.20 \\
\hline 45 & 1.01 & 1.68 & 2.01 & 2.06 & 2.69 & 3.18 \\
\hline 50 & 1.01 & 1.68 & 2.01 & 2.05 & 2.68 & 3.16 \\
\hline 100 & 1.005 & 1.660 & 1.984 & 2.025 & 2.626 & 3.077 \\
\hline$\infty$ & 1.000 & 1.645 & 1.960 & 2.000 & 2.576 & 3.000 \\
\hline
\end{tabular}


Appendix C

NIST Technical Communications Program

\section{APPENDIX E \\ STATEMENTS OF UNCERTAINTY ASSOCIATED WITH MEASUREMENT RESULTS}

\begin{abstract}
A measurement result is complete only when accompanied by a quantitative statement of its uncertainty. This policy requires that NIST measurement results be accompanied by such statements and that a uniform approach to expressing measurement uncertainty be followed.
\end{abstract}

\section{Background}

Since the early 1980s, an international consensus has been developing on a uniform approach to the expression of uncertainty in measurement. Many of NIST's sister national standards laboratories as well as a number of important metrological organizations, including the Western European Calibration Cooperation (WECC) and EUROMET, have adopted the approach recommended by the International Committee for Weights and Measures (CIPM) in 1981 [1] and reaffirmed by the CIPM in 1986 [2].

Equally important, the CIPM approach has come into use in a significant number of areas at NIST and is also becoming accepted in U.S. industry. For example, the National Conference of Standards Laboratories (NCSL) is using it to develop a Recommended Practice on measurement uncertainty for NCSL member laboratories.

The CIPM approach is based on Recommendation INC-1 (1980) of the Working Group on the Statement of Uncertainties [3]. This group was convened in 1980 by the International Bureau of Weights and Measures (BIPM) in response to a request by the CIPM. More recently, at the request of the CIPM, a joint BIPM/IEC/ISO/OIML working group developed a comprehensive reference document on the general application of the CIPM approach titled Guide to the Expression of Uncertainty in Measurement [4] (IEC: International Electrotechnical
Commission; ISO: International Organization for Standardization; OIML: International Organization of Legal Metrology). The development of the Guide is providing further impetus to the worldwide adoption of the CIPM approach.

\section{Policy}

All NIST measurement results are to be accompanied by quantitative statements of uncertainty. To ensure that such statements are consistent with each other and with present international practice, this NIST policy adopts in substance the approach to expressing measurement uncertainty recommended by the International Committee for Weights and Measures (CIPM). The CIPM approach as adapted for use by NIST is:

1) Standard Uncertainty: Represent each component of uncertainty that contributes to the uncertainty of the measurement result by an estimated standard deviation $u_{i}$, termed standard uncertainty, equal to the positive square root of the estimated variance $u_{i}^{2}$.

2) Combined Standard Uncertainty: Determine the combined standard uncertainty $u_{\mathrm{c}}$ of the measurement result, taken to represent the estimated standard deviation of the result, by combining the individual standard uncertainties $u_{i}$ (and covariances as appropriate) using the usual "root-sum-of-squares" method, or equivalent established and documented methods.

Commonly, $u_{\mathrm{c}}$ is used for reporting results of determinations of fundamental constants, fundamental metrological research, and international comparisons of realizations of SI units. 
3) Expanded Uncertainty: Determine an expanded uncertainty $U$ by multiplying $u_{c}$ by a coverage factor $k$ : $U=k u_{\mathrm{c}}$. The purpose of $U$ is to provide an interval $y-U$ to $y+U$ about the result $y$ within which the value of $Y$, the specific quantity subject to measurement and estimated by $y$, can be asserted to lie with a high level of confidence. Thus one can confidently assert that $y-U \leq Y \leq y+U$, which is commonly written as $Y=y \pm U$.

Use expanded uncertainty $U$ to report the results of all NIST measurements other than those for which $u_{c}$ has traditionally been employed. To be consistent with current international practice, the value of $k$ to be used at NIST for calculating $U$ is, by convention, $k=2$. Values of $k$ other than 2 are only to be used for specific applications dictated by established and documented requirements.

4) Reporting Uncertainty: Report $U$ together with the coverage factor $k$ used to obtain it, or report $u_{\mathrm{c}}$.

When reporting a measurement result and its uncertainty, include the following information in the report itself or by referring to a published document:

- A list of all components of standard uncertainty, together with their degrees of freedom where appropriate, and the resulting value of $u_{c}$. The components should be identified according to the method used to estimate their numerical values:
A. those which are evaluated by statistical methods,
B. those which are evaluated by other means.

- A detailed description of how each component of standard uncertainty was evaluated.
- A description of how $k$ was chosen when $k$ is not taken equal to 2 .

It is often desirable to provide a probability interpretation, such as a level of confidence, for the interval defined by $U$ or $u_{\mathrm{c}}$. When this is done, the basis for such a statement must be given.
Additional guidance on the use of the CIPM approach at NIST may be found in Guidelines for Evaluating and Expressing the Uncertainty of NIST Measurement Results [5]. A more detailed discussion of the CIPM approach is given in the Guide to the Expression of Uncertainty in Measurement [4]. Classic expositions of the statistical evaluation of measurement processes are given in references [6-8].

\section{Responsibilities}

a. Operating Unit Directors are responsible for compliance with this policy.

b. The Statistical Engineering Division, Computing and Applied Mathematics Laboratory, is responsible for providing technical advice on statistical methods for evaluating and expressing the uncertainty of NIST measurement results.

c. NIST Editorial Review Boards are responsible for ensuring that statements of measurement uncertainty are included in NIST publications and other technical outputs under their jurisdiction which report measurement results and that such statements are in conformity with this policy.

d. The Calibrations Advisory Group is responsible for ensuring that calibration and test reports and other technical outputs under its jurisdiction are in compliance with this policy.

e. The Standard Reference Materials and Standard Reference Data programs are responsible for ensuring that technical outputs under their jurisdiction are in compliance with this policy.

f. Authors, as part of the process of preparing manuscripts and other technical outputs, are responsible for formulating measurement uncertainty statements consistent with this policy. These statements must be present in drafts submitted for NIST review and approval. 


\section{Exceptions}

It is understood that any valid statistical method that is technically justified under the existing circumstances may be used to determine the equivalent of $u_{i}, u_{\mathrm{c}}$, or $U$. Further, it is recognized that international, national, or contractual agreements to which NIST is a party may occasionally require deviation from this policy. In both cases, the report of uncertainty must document what was done and why.

\section{References Cited}

[1] CIPM, BIPM Proc. Verb. Com. Int. Poids et Mesures 49, 8-9, 26 (1981) (in French); P. Giacomo, “News from the BIPM,” Metrologia 18, 41-44 (1982).

[2] CIPM, BIPM Proc.-Verb. Com. Int. Poids et Mesures 54, 14, 35 (1986) (in French); P. Giacomo, “News from the BIPM," Metrologia 24, 45-51 (1987).

[3] R. Kaarls, "Rapport du Groupe de Travail sur l'Expression des Incertitudes au Comité International des Poids et Mesures," Proc.-Verb. Com. Int. Poids et Mesures 49, A1-A12 (1981) (in French); P. Giacomo, "News from the BIPM," Metrologia 17, 69-74 (1981). (Note that the final English-language version of Recommendation INC1 (1980), published in an internal BIPM report, differs slightly from that given in the latter reference but is consistent with the authoritative French-language version given in the former reference.)

[4] ISO, Guide to the Expression of Uncertainty in Measurement, prepared by ISO Technical Advisory Group 4 (TAG 4), Working Group 3 (WG 3),
October 1993. ISO/TAG 4 has as its sponsors the BIPM, IEC, IFCC (International Federation of Clinical Chemistry), ISO, IUPAC (International Union of Pure and Applied Chemistry), IUPAP (International Union of Pure and Applied Physics), and OIML. Although the individual members of WG 3 were nominated by the BIPM, IEC, ISO, or OIML, the Guide is published by ISO in the name of all seven organizations. NIST staff members may obtain a single copy of the Guide from the NIST Calibration Program.

[5] B. N. Taylor and C. E. Kuyatt, Guidelines for Evaluating and Expressing the Uncertainty of NIST Measurement Results, NIST Technical Note 1297, prepared under the auspices of the NIST Ad Hoc Committee on Uncertainty Statements (U.S. Government Printing Office, Washington, DC, January 1993).

[6] C. Eisenhart, "Realistic Evaluation of the Precision and Accuracy of Instrument Calibration Systems," J. Res. Natl. Bur. Stand. (U.S.) 67C, 161-187 (1963). Reprinted, with corrections, in Precision Measurement and Calibration: Statistical Concepts and Procedures, NBS Special Publication 300, Vol. I, H. H. Ku, Editor (U.S. Government Printing Office, Washington, DC, 1969), pp. 21-48.

[7] J. Mandel, The Statistical Analysis of Experimental Data (Interscience-Wiley Publishers, New York, NY, 1964, out of print; corrected and reprinted, Dover Publishers, New York, NY, 1984).

[8] M. G. Natrella, Experimental Statistics, NBS Handbook 91 (U.S. Government Printing Office, Washington, DC, 1963; reprinted October 1966 with corrections). 


\section{Appendix D}

\section{Clarification and Additional Guidance}

As indicated in our Preface to this second (1994) edition of TN 1297, Appendix D has been added to clarify and provide additional guidance on a number of topics. It was prepared in response to questions asked since the publication of the first (1993) edition.

\section{D.1 Terminology}

D.1.1 There are a number of terms that are commonly used in connection with the subject of measurement uncertainty, such as accuracy of measurement, reproducibility of results of measurements, and correction. One can avoid confusion by using such terms in a way that is consistent with other international documents.

Definitions of many of these terms are given in the International Vocabulary of Basic and General Terms in Metrology [D.1], the title of which is commonly abbreviated VIM. The VIM and the Guide may be viewed as companion documents inasmuch as the VIM, like the Guide, was developed by ISO Technical Advisory Group 4 (TAG 4), in this case by its Working Group 1 (WG 1); and the VIM, like the Guide, was published by ISO in the name of the seven organizations that participate in the work of TAG 4. Indeed, the Guide contains the VIM definitions of 24 relevant terms. For the convenience of the users of TN 1297, the definitions of eight of these terms are included here.

NOTE - In the following definitions, the use of parentheses around certain words of some terms means that the words may by omitted if this is unlikely to cause confusion. The VIM identification number for a particular term is shown in brackets after the term.

\section{D.1.1.1 accuracy of measurement [VIM 3.5]}

closeness of the agreement between the result of a measurement and the value of the measurand

\author{
NOTES \\ 1 "Accuracy" is a qualitative concept. \\ 2 The term precision should not be used for "accuracy."
}

\section{TN 1297 Comments:}

1 The phrase "a true value of the measurand" (or sometimes simply "a true value"), which is used in the VIM definition of this and other terms, has been replaced here and elsewhere with the phrase "the value of the measurand." This has been done to reflect the view of the Guide, which we share, that "a true value of a measurand" is simply the value of the measurand. (See subclause D.3.5 of the Guide for further discussion.)

2 Because "accuracy" is a qualitative concept, one should not use it quantitatively, that is, associate numbers with it; numbers should be associated with measures of uncertainty instead. Thus one may write "the standard uncertainty is $2 \mu \Omega$ " but not "the accuracy is $2 \mu \Omega$."

3 To avoid confusion and the proliferation of undefined, qualitative terms, we recommend that the word "inaccuracy" not be used.

4 The VIM does not give a definition for "precision" because of the many definitions that exist for this word. For a discussion of precision, see subsection D.1.2.

\section{D.1.1.2 repeatability (of results of measurements) [VIM} 3.6]

closeness of the agreement between the results of successive measurements of the same measurand carried out under the same conditions of measurement

\section{NOTES}

1 These conditions are called repeatability conditions

2 Repeatability conditions include:

- the same measurement procedure

- the same observer

- the same measuring inscrument, used under the same conditions

- the same location

- repetition over a shor period of time.

3 Repeatability may be expressed quantitatively in terms of the dispersion characteristics of the results.

\section{D.1.1.3 reproducibility (of results of measurements)} [VIM 3.7]

closeness of the agreement between the results of measurements of the same measurand carried out under changed conditions of measurement

\section{NOTES}

1 A valid statement of reproducibility requires specification of the conditions changed.

2 The changed condinions may include:

- principle of measurement

- method of measurement

- observer 
- measuring instrument

- reference standard

- location

- conditions of use

- time.

3 Reproducibility may be expressed quantitatively in terms of the dispersion characteristics of the results.

4 Results are here usually understood to be corrected results.

\section{D.1.1.4 error (of measurement) [VIM 3.10]}

result of a measurement minus the value of the measurand

\section{NOTES}

1 Since the value of the measurand cannot be determined, in practice a conventional value is [sometimes] used (see [VIM] 1.19 and 1.20).

2 When it is uecessary to distinguish "error" from "relative error," the former is sometimes called absolute error of measurement. This should not be confused with absolute value of error, which is the modulus of the error.

\section{TN 1297 Comments:}

1 As pointed out in the Guide, if the result of a measurement depends on the values of quantities other than the measurand, the errors of the measured values of these quantities contribute to the error of the result of the measurement.

2 In general, the error of measurement is unknown because the value of the measurand is unknown. However, the uncertainty of the result of a measurement may be evaluated.

3 As also pointed out in the Guide, if a device (taken to include measurement standards, reference materials, etc.) is tested through a comparison with a known reference standard and the uncertainties associated with the standard and the comparison procedure can be assumed to be negligible relative to the required uncertainty of the test, the comparison may be viewed as determining the error of the device.

\section{D.1.1.5 random error [VIM 3.13]}

result of a measurement minus the mean that would result from an infinite number of measurements of the same measurand carried out under repeatability conditions

\section{NOTES}

1 Random error is equal to error minus systematic error.
2 Because only a finite number of measurements can be made, it is possible to determine only an estimate of random error.

TN 1297 Comment:

The concept of random error is also often applied when the conditions of measurement are changed (see subsection D.1.1.3). For example, one can conceive of obtaining measurement results from many different observers while holding all other conditions constant, and then calculating the mean of the results as well as an appropriate measure of their dispersion (e.g., the variance or standard deviation of the results).

\section{D.1.1.6 systematic error [VIM 3.14]}

mean that would result from an infinite number of measurements of the same measurand carried out under repeatability conditions minus the value of the measurand

\section{NOTES}

1 Systematic error is equal to error minus random error.

2 Like the value of the measurand, systematic error and its causes cannot be completely known.

3 For a measuring instrument, see "bias" ([VIM] 5.25).

\section{TN 1297 Comments:}

1 As pointed out in the Guide, the error of the result of a measurement may often be considered as arising from a number of random and systematic effects that contribute individual components of error to the error of the result.

2 Although the term bias is often used as a synonym for the term systematic error, because systematic error is defined in a broadly applicable way in the VIM while bias is defined only in connection with a measuring instrument, we recommend the use of the term systematic error.

\section{D.1.1.7 correction [VIM 3.15]}

value added algebraically to the uncorrected result of a measurement to compensate for systematic error

\section{NOTES}

1 The correction is equal to the negative of the estimated systematic error.

2 Since the systematic error cannot be known perfectly, the compensation cannot be complete.

\section{D.1.1.8 correction factor [VIM 3.16]}

numerical factor by which the uncorrected result of a measurement is multiplied to compensate for systematic error 
NOTE - Since the systematic error cannot be known perfectly, the compensation cannot be complete,

\section{D.1.2 As indicated in subsection D.1.1.1, TN 1297} comment 4 , the VIM does not give a definition for the word "precision." However, ISO 3534-1 [D.2] defines precision to mean "the closeness of agreement between independent test results obtained under stipulated conditions." Further, it views the concept of precision as encompassing both repeatability and reproducibility (see subsections D.1.1.2 and D.1.1.3) since it defines repeatability as "precision under repeatability conditions," and reproducibility as "precision under reproducibility conditions." Nevertheless, precision is often taken to mean simply repeatability..

The term precision, as well as the terms accuracy, repeatability, reproducibility, variability, and uncertainty, are examples of terms that represent qualitative concepts and thus should be used with care. In particular, it is our strong recommendation that such terms not be used as synonyms or labels for quantitative estimates. For example, the statement "the precision of the measurement results, expressed as the standard deviation obtained under repeatability conditions, is $2 \mu \Omega^{n}$ is acceptable, but the statement "the precision of the measurement results is 2 $\mu \Omega$ " is not. (See also subsection D.1.1.1, TN 1297 comment 2.)

Although reference [D.2] states that "The measure of precision is usually expressed in terms of imprecision and computed as a standard deviation of the test results," we recommend that to avoid confusion, the word "imprecision" not be used; standard deviation and standard uncertainty are preferred, as appropriate (see subsection D.1.5).

It should also be borne in mind that the NIST policy on expressing the uncertainty of measurement results normally requires the use of the terms standard uncertainty, combined standard uncertainty, expanded uncertainty, or their "relative" forms (see subsection D.1.4), and the listing of all components of standard uncertainty. Hence the use of terms such as accuracy, precision, and bias should normally be as adjuncts to the required terms and their relationship to the required terms should be made clear. This situation is similar to the NIST policy on the use of units that are not part of the SI: the SI units must be stated first, with the units that are not part of the SI in parentheses (see subsection D.6.2).

D.1.3 The designations " $\mathrm{A}$ " and " $\mathrm{B}$ " apply to the two distinct methods by which uncertainty components may be evaluated. However, for convenience, a standard uncertainty obtained from a Type A evaluation may be called a Type $A$ standard uncertainty; and a standard uncertainty obtained from a type B evaluation may be called a Type $B$ standard uncertainty. This means that:

(1) " $A$ " and " $B$ " have nothing to do with the traditional terms "random" and "systematic";

(2) there are no "Type A errors" or "Type B errors"; and

(3) "Random uncertainty" (i.e., an uncertainty component that arises from a random effect) is not a synonym for Type A standard uncertainty; and "systematic uncertainty" (i.e., an uncertainty component that arises from a correction for a systematic error) is not a synonym for Type B standard uncertainty.

In fact, we recommend that the terms "random uncertainty" and "systematic uncertainty" be avoided because the adjectives "random" and "systematic," while appropriate modifiers for the word "error," are not appropriate modifiers for the word "uncertainty" (one can hardly imagine an uncertainty component that varies randomly or that is systematic).

D.1.4 If $u\left(x_{i}\right)$ is a standard uncertainty, then $u\left(x_{i}\right) /\left|x_{i}\right|$, $x_{i} \neq 0$, is the corresponding relative standard uncertainty; if $u_{\mathrm{c}}(y)$ is a combined standard uncertainty, then $u_{\mathrm{c}}(y) /|y|$, $y \neq 0$, is the corresponding relative combined standard uncertainty; and if $U=k u_{c}(y)$ is an expanded uncertainty, then $U /|y|, y \neq 0$, is the corresponding relative expanded uncertainty. Such relative uncertainties may be readily indicated by using a subscript " $\mathrm{r}$ " for the word "relative." Thus $u_{\mathrm{r}}\left(x_{i}\right) \equiv u\left(x_{i}\right) /\left|x_{i}\right|, u_{\mathrm{c}, \mathrm{r}}(y) \equiv u_{\mathrm{c}}(y) /|y|$, and $U_{\mathrm{r}} \equiv U /|y|$.

D.1.5 As pointed out in subsection D.1.2, the use of the terms standard uncertainty, combined standard uncertainty, expanded uncertainty, or their equivalent "relative" forms (see subsection D.1.4), is normally required by NIST policy. Alternate terms should therefore play a subsidiary role in any NIST publication that reports the result of a measurement and its uncertainty. However, since it will take some time before the meanings of these terms become well known, they should be defined at the beginning of a paper or when first used. In the latter case, this may be done by writing, for example, "the standard uncertainty (estimated standard deviation) is $u(R)=2 \mu \Omega$ "; or "the expanded uncertainty (coverage factor $k=2$ and thus a twostandard-deviation estimate) is $U=4 \mu \Omega$. $^{n}$

It should also be recognized that, while an estimated standard deviation that is a component of uncertainty of a 
measurement result is properly called a "standard uncertainty," not every estimated standard deviation is necessarily a standard uncertainty.

D.1.6 Words such as "estimated" or "limits of" should normally not be used to modify "standard uncertainty," "combined standard uncertainty," "expanded uncertainty," the "relative" forms of these terms (see subsection D.1.4), or more generally "uncertainty." The word "uncertainty," by its very nature, implies that the uncertainty of the result of a measurement is an estimate and generally does not have well-defined limits.

D.1.7 The phrase "components of uncertainty that contribute to the uncertainty of the measurement result ${ }^{n}$ can have two distinct meanings. For example, if the input estimates $x_{i}$ are uncorrelated, Eq. (A-3) of Appendix A may be written as

$$
u_{c}^{2}=\sum_{i=1}^{N}\left[c_{i} u\left(x_{i}\right)\right]^{2} \equiv \sum_{i=1}^{N} u_{i}^{2}(y)
$$

where $c_{i} \equiv \partial f / \partial x_{i}$ and $u_{i}(y) \equiv\left|c_{i}\right| u\left(x_{i}\right)$.

In Eq. (D-1), both $u\left(x_{i}\right)$ and $u_{i}(y)$ can be considered components of uncertainty of the measurement result $y$. This is because the $u\left(x_{i}\right)$ are the standard uncertainties of the input estimates $x_{i}$ on which the output estimate or measurement result $y$ depends; and the $u_{i}(y)$ are the standard uncertainties of which the combined standard uncertainty $u_{\mathrm{c}}(y)$ of the measurement result $y$ is composed. In short, both $u\left(x_{i}\right)$ and $u_{i}(y)$ can be viewed as components of uncertainty that give rise to the combined standard uncertainty $u_{c}(y)$ of the measurement result $y$. This implies that in subsections 2.4 to $2.6,4.4$ to 4.6 , and 6.6 ; in 1 ) and 2) of section 2 of Appendix $C$; and in section 4 of Appendix $\mathrm{C}$, the symbols $u_{i}$, $s_{i}$, or $u_{j}$ may be viewed as representing either $u\left(x_{i}\right)$ or $u_{i}(y)$.

When one gives the components of uncertainty of a result of a measurement, it is recommended that one also give the standard uncertainties $u\left(x_{i}\right)$ of the input estimates $x_{i}$, the sensitivity coefficients $c_{i} \equiv \partial f / \partial x_{i}$, and the standard uncertainties $u_{i}(y)=\left|c_{i}\right| u\left(x_{i}\right)$ of which the combined standard uncertainty $u_{\mathrm{c}}(y)$ is composed (so-called standard uncertainty components of combined standard uncertainty).

D.1.8 The VIM gives the name "experimental standard deviation of the mean" to the quantity $s\left(\bar{X}_{i}\right)$ of Eq. (A-5) of Appendix A of this Technical Note, and the name "experimental standard deviation" to the quantity $s\left(X_{i, k}\right)=$ $\sqrt{n} s\left(\bar{X}_{i}\right)$. We believe that these are convenient, descriptive terms, and therefore suggest that NIST authors consider using them.

\section{D.2 Identification of uncertainty components}

D.2.1 The NIST policy on expressing measurement uncertainty states that all components of standard uncertainty "should be identified according to the method used to estimate their numerical values: $\mathrm{A}$. those which are evaluated by statistical methods, B. those which are evaluated by other means."

Such identification will usually be readily apparent in the "detailed description of how each component of standard uncertainty was evaluated" that is required by the NIST policy. However, such identification can also be given in a table which lists the components of standard uncertainty. Tables D.1 and D.2, which are based on the end-gauge

Table D.1 - Uncertainty Budget: End-Gauge Calibration

\begin{tabular}{|c||c|}
\hline \multicolumn{1}{|c||}{$\begin{array}{c}\text { Source of } \\
\text { uncertainty }\end{array}$} & $\begin{array}{c}\text { Standard } \\
\text { uncertainty } \\
(\mathrm{nm})\end{array}$ \\
\hline \hline $\begin{array}{c}\text { Calibration of standard end } \\
\text { gauge }\end{array}$ & $25(\mathrm{~B})$ \\
\hline $\begin{array}{c}\text { Measured difference between } \\
\text { end gauges: } \\
\text { repeated observations } \\
\text { random effects of } \\
\text { comparator }\end{array}$ & $5.8(\mathrm{~A})$ \\
\hline $\begin{array}{c}\text { systematic effects of } \\
\text { comparator }\end{array}$ & 3.9 (A) \\
\hline $\begin{array}{c}\text { Thermal expansion of } \\
\text { standard end gauge }\end{array}$ & 6.7 (B) \\
\hline $\begin{array}{c}\text { Temperature of test bed: } \\
\text { mean temperature of bed } \\
\text { cyclic variation of } \\
\text { temperature of room }\end{array}$ & $16.6(\mathrm{~B})$ \\
\hline $\begin{array}{c}\text { Difference in expansion } \\
\text { coefficients of end gauges }\end{array}$ & $5.8(\mathrm{~A})$ \\
\hline $\begin{array}{c}\text { Difference in temperatures of } \\
\text { end gauges }\end{array}$ & $10.2(\mathrm{~B})$ \\
\hline \hline Combined standard uncertainty: $u_{\mathrm{c}}(l)=34 \mathrm{~nm}$ \\
\hline
\end{tabular}




\section{Table D.2 - Uncertainty Budget: End-Gauge Calibration}

\begin{tabular}{|c|c|c|c|c|}
\hline \multirow[t]{2}{*}{$\begin{array}{l}\text { Source of } \\
\text { uncertainty }\end{array}$} & \multicolumn{2}{|c|}{$\begin{array}{c}\text { Standard uncertainties } \\
\text { from random effects } \\
\text { in the current measurement process } \\
(\mathrm{nm})\end{array}$} & \multicolumn{2}{|c|}{$\begin{array}{l}\text { Standard uncertainties } \\
\text { from systematic effects } \\
\text { in the current measurement process } \\
(\mathrm{nm})\end{array}$} \\
\hline & $\begin{array}{l}\text { Type A } \\
\text { evaluation }\end{array}$ & $\begin{array}{l}\text { Type B } \\
\text { evaluation }\end{array}$ & $\begin{array}{l}\text { Type A } \\
\text { evaluation }\end{array}$ & $\begin{array}{l}\text { Type B } \\
\text { evaluation }\end{array}$ \\
\hline Calibration of standard end gauge & & & & 25 \\
\hline $\begin{array}{l}\text { Measured difference between end } \\
\text { gauges: } \\
\text { repeated observations } \\
\text { random effects of comparator } \\
\text { systematic effects of } \\
\text { comparator }\end{array}$ & 5.8 & & 3.9 & 6.7 \\
\hline $\begin{array}{l}\text { Thermal expansion of standard } \\
\text { end gauge }\end{array}$ & & & & 1.7 \\
\hline $\begin{array}{l}\text { Temperature of test bed: } \\
\text { mean temperature of bed } \\
\text { cyclic variation of temperature } \\
\text { of room }\end{array}$ & 5.8 & & & 10.2 \\
\hline $\begin{array}{l}\text { Difference in expansion } \\
\text { coefficients of end gauges }\end{array}$ & & & & 2.9 \\
\hline $\begin{array}{l}\text { Difference in temperatures of end } \\
\text { gauges }\end{array}$ & & 16.6 & & \\
\hline
\end{tabular}

calibration example of the Guide (subclause H.1), are two examples of such tables.

D.2.2 In Table D.1, the method used to evaluate a particular standard uncertainty is shown in parentheses. In Table D.2, the method is indicated by using different columns. The latter table also shows how one can indicate whether a component arose from a random effect in the current measurement process or from a systematic effect in the current measurement process, assuming that such information is believed to be useful to the reader.

If a standard uncertainty is obtained from a source outside of the current measurement process and the nature of its individual components are unknown (which will often be the case), it may be classified as having been obtained from a Type $\mathrm{B}$ evaluation. If the standard uncertainty from an outside source is known to be composed of components obtained from both Type A and Type B evaluations but the magnitudes of the individual components are unknown, then one may indicate this by using $(A, B)$ rather than $(B)$ in a table such as D.1.

On the other hand, a standard uncertainty known to be composed of components obtained from Type A evaluations alone should be classified as a Type A standard uncertainty, while a standard uncertainty known to be composed of components obtained from Type B evaluations alone should be classified as a Type B standard uncertainty.

In this same vein, if the combined standard uncertainty $u_{c}(y)$ of the measurement result $y$ is obtained from Type A standard uncertainties (and covariances) only, it too may be considered Type A, even though no direct observations 
were made of the measurand $Y$ of which the measurement result $y$ is an estimate. Similarly, if a combined standard uncertainty is obtained from Type B standard uncertainties (and covariances) only, it too may be considered Type B.

\section{D.3 Equation (A-2)}

D.3.1 In the most general sense, Eq. (A-2) of Appendix A of this Technical Note,

$$
y=f\left(x_{1}, x_{2}, \ldots, x_{N}\right),
$$

is a symbolic representation of the procedure (or algorithm) used to obtain the output estimate $y$, which is the result of the measurement, from the individual input estimates $x_{i}$. For example, some of the $x_{i}$ may themselves depend on additional input estimates:

$$
\begin{aligned}
& x_{1}=g_{1}\left(w_{1}, w_{2}, \ldots, w_{K}\right) \\
& x_{2}=g_{2}\left(z_{1}, z_{2}, \ldots, z_{L}\right)
\end{aligned}
$$

etc.

Or the output estimate $y$ may be expressible simply as

$$
y=x+C_{1}+C_{2}+\ldots+C_{M},
$$

where the $C_{i}$ are corrections, for example, for the operator, for the ambient temperature, for the laboratory, etc. Some or all of the $C_{i}$ may be estimated to be near zero based on the available information, but they can still have standard uncertainties that are large enough to contribute significantly to the combined standard uncertainty of the measurement result and which therefore must be evaluated.

NOTE - In some situations, a correction for a particular effect and its standard uncertainty are estimated to be negligible relative to the required combined standard uncertainty of the measurement result, and for added confidence, an experimental test is carried out that confirms the estimate but the standard uncertainty of the test result is not negligible. In such cases, if other evidence indicates that the estimate is in fact reliable, the standard uncertainty of the test result need not be included in the uncertainty budget and both the correction and its standard uncertainty can be taken as negligible.

D.4 Measurand defined by the measurement method; characterization of test methods; simple calibration

D.4.1 The approach to evaluating and expressing the uncertainty of a measurement result on which the NIST policy and this Technical Note are based is applicable to evaluating and expressing the uncertainty of the estimated value of a measurand that is defined by a standard method of measurement. In this case, the uncertainty depends not only on the repeatability and reproducibility of the measurement results (see subsections D.1.1.2 and D.1.1.3), but also on how well one believes the standard measurement method has been implemented. (See example H.6 of the Guide.)

When reporting the estimated value and uncertainty of such a measurand, one should always make clear that the measurand is defined by a particular method of measurement and indicate what that method is. One should also give the measurand a name which indicates that it is defined by a measurement method, for example, by adding a modifier such as "conventional." (See also subsection D.6.1)

D.4.2 There are national as well as international standards that discuss the characterization of test methods by interlaboratory comparisons. Execution of test methods according to these standards, both in the characterization stage and in subsequent measurement programs, often calls for the expression of uncertainties in terms of defined measures of repeatability and reproducibility. When NIST authors participate in such characterization or measurement programs, NIST policy allows for the results to be expressed as required by the relevant standards (see Appendix C, section 4). However, when NIST authors document work according to such standards, they should consider making the resulting publication understandable to a broad audience. This might be achieved in part by giving definitions of the terms used, perhaps in a footnote. If possible, NIST authors should relate these terms to those of this Technical Note and of the Guide.

If a test method is employed at NIST to obtain measurement results for reasons other than those described above, it is expected that the uncertainties of these measurement results will be evaluated and reported according to section 2 of the NIST policy (see Appendix C). This would be the case, for example, if measurement results from a characterized test method are compared to those from a new method of measurement which has not been characterized by interlaboratory comparisons.

D.4.3 When an unknown standard is calibrated in terms of a known reference standard at lower levels of the measurement hierarchy, the uncertainty of the result of calibration may have as few as two components: a single Type A standard uncertainty evaluated from the pooled experimental standard deviation that characterizes the calibration process; and a single Type B (or possibly 
Type A) standard uncertainty obtained from the calibration certificate of the known reference standard.

NOTE - The possibility of unsuspected systematic effects in the calibration process used to calibrate the unknown standard should, however, not be overlooked.

\section{D.5 $t_{p}$ and the quantile $t_{1-\alpha}$}

D.5.1 As pointed out in the Guide, the $t$-distribution is often tabulated in quantiles. That is, values of the quantile $t_{l-\alpha}$ are given, where $1-\alpha$ denotes the cumulative probability and the relation

$$
1-\alpha=\int_{-\infty}^{t_{1}-\alpha} f(t, v) \mathrm{d} t
$$

defines the quantile, where $f$ is the probability density function of $t$. Thus $t_{p}$ of this Technical Note and of the Guide and $t_{1-\alpha}$ are related by $p=1-2 \alpha$. For example, the value of the quantile $t_{0.975}$, for which $1-\alpha=0.975$ and $\alpha=0.025$, is the same as $t_{p}(v)$ for $p=0.95$. It should be noted, however, that in reference [D.2] the symbol $p$ is used for the cumulative probability $1-\alpha$, and the resulting $t_{p}(v)$ is called the "quantile of order $p$ of the $t$ variable with $v$ degrees of freedom." Clearly, the values of $t_{p}(v)$ defined in this way differ from the values of $t_{p}(v)$ defined as in this Technical Note and in the Guide, and given in Table B.1 (which is of the same form as that given in reference [10]). Thus, one must use tables of tabulated values of $t_{p}(v)$ with some care.

\section{D.6 Uncertainty and units of the SI; proper use of the} SI and quantity and unit symbols

D.6.1 As pointed out in the Guide, the result of a measurement is sometimes expressed in terms of the adopted value of a measurement standard or in terms of a conventional reference value rather than in terms of the relevant unit of the SI. (This is an example of a situation in which all significant components of uncertainty are not taken into account.) In such cases the magnitude of the uncertainty ascribable to the measurement result may be significantly smaller than when that result is expressed in the relevant SI unit. This practice is not disallowed by the NIST policy, but it should always be made clear when the practice is being followed. In addition, one should always give some indication of the values of the components of uncertainty not taken into account. The following example is taken from the Guide. (See also subsection D.4.1.)

EXAMPLE - A high-qualiry Zener volage standard is calibrated by comparison with a Josephson effect voltage reference based on the conventional value of the Josephson constant recommended for international use by the CIPM. The relative combined standard uncertainty $u_{c}\left(V_{S}\right) / V_{S}$ of the calibrated potential difference $V_{S}$ of the Zener standard is $2 \times 10^{-8}$ when $V_{S}$ is reported in terms of the conventional value, but $\mu_{\mathrm{S}}\left(V_{\mathrm{S}}\right) / V_{\mathrm{S}}$ is $4 \times 10^{-7}$ when $V_{\mathrm{S}}$ is reported in terms of the SI unit of potennial difference, the voit $(V)$, because of the additional uncertainty associated with the SI value of the Josephson constant.

D.6.2 NIST Special Publication 811, 1995 Edition [D.3], gives guidance on the use of the SI and on the rules and style conventions regarding quantity and unit symbols. In particular, it elaborates upon the NIST policy regarding the SI and explains why abbreviarions such as ppm and ppb and terms such as normality and molarity should not be used. NIST authors should consult NIST SP 811 if they have any questions concerning the proper way to express the values of quantities and their uncertainties.

\section{D.7 References}

[D.1] ISO, International Vocabulary of Basic and General Terms in Metrology, second edition (International Organization for Standardization. Geneva, Switzerland, 1993). This document (abbreviated VIM) was prepared by ISO Technical Advisory Group 4 (TAG 4), Working Group 1 (WG 1). ISO/TAG 4 has as its sponsors the BIPM, IEC, IFCC (International Federation of Clinical Chemistry), ISO, IUPAC (International Union of Pure and Applied Chemistry), IUPAP (International Union of Pure and Applied Physics), and OIML. The individual members of WG 1 were nominated by BIPM, IEC, IFCC, ISO, IUPAC IUPAP, or OIML, and the document is published by ISO in the name of all seven organizations. NIST staff members may obtain a single copy of the VIM from the NIST Calibration Program.

[D.2] ISO 3534-1:1993, Statistics - Vocabulary and symbols - Part 1: Probability and general statistical terms (International Organizarion for Standardization, Geneva, Switzerland, 1993).

[D.3] B. N. Taylor, Guide for the Use of the International System of Units (SI), NIST Special Publication 811, 1995 Edition (U.S. Government Printing Office, Washington, DC, April 1995). 


Journal of Research of the National Institute of Standards and Technology-Reports NIST research and development in those disciplines of the physical and engineering sciences in which the Institute is active. These include physics, chemistry, engineering, mathematics, and computer sciences. Papers cover a broad range of subjects, with major emphasis on measurement methodology and the basic technology underlying standardization. Also included from time to time are survey articles on topics closely related to the Institute's technical and scientific programs. Issued six times a year.

\section{Nonperiodicals}

Monographs-Major contributions to the technical literature on various subjects related to the Institute's scientific and technical activities.

Handbooks-Recommended codes of engineering and industrial practice (including safety codes) developed in cooperation with interested industries, professional organizations, and regulatory bodies.

Special Publications-Include proceedings of conferences sponsored by NIST, NIST annual reports, and other special publications appropriate to this grouping such as wall charts, pocket cards, and bibliographies.

National Standard Reference Data Series-Provides quantitative data on the physical and chemical properties of materials, compiled from the world's literature and critically evaluated. Developed under a worldwide program coordinated by NIST under the authority of the National Standard Data Act (Public Law 90-396). NOTE: The Journal of Physical and Chemical Reference Data (JPCRD) is published bimonthly for NIST by the American Chemical Society (ACS) and the American Institute of Physics (AIP). Subscriptions, reprints, and supplements are available from ACS, 1155 Sixteenth St., NW, Washington, DC 20056.

Building Science Series-Disseminates technical information developed at the Institute on building materials, components, systems, and whole structures. The series presents research results, test methods, and performance criteria related to the structural and environmental functions and the durability and safety characteristics of building elements and systems.

Technical Notes-Studies or reports which are complete in themselves but restrictive in their treatment of a subject. Analogous to monographs but not so comprehensive in scope or definitive in treatment of the subject area. Often serve as a vehicle for final reports of work performed at NIST under the sponsorship of other government agencies.

Voluntary Product Standards-Developed under procedures published by the Department of Commerce in Part 10, Title 15, of the Code of Federal Regulations. The standards establish nationally recognized requirements for products, and provide all concerned interests with a basis for common understanding of the characteristics of the products. NIST administers this program in support of the efforts of private-sector standardizing organizations.

Order the following NIST publications-FIPS and NISTIRs—from the National Technical Information Service, Springfield, VA 22161.

Federal Information Processing Standards Publications (FIPS PUB)-Publications in this series collectively constitute the Federal Information Processing Standards Register. The Register serves as the official source of information in the Federal Government regarding standards issued by NIST pursuant to the Federal Property and Administrative Services Act of 1949 as amended, Public Law 89-306 (79 Stat. 1127), and as implemented by Executive Order 11717 (38 FR 12315, dated May 11, 1973) and Part 6 of Title 15 CFR (Code of Federal Regulations).

NIST Interagency or Internal Reports (NISTIR) - The series includes interim or final reports on work performed by NIST for outside sponsors (both government and nongovernment). In general, initial distribution is handled by the sponsor; public distribution is handled by sales through the National Technical Information Service, Springfield, VA 22161, in hard copy, electronic media, or microf iche form. NISTIR's may also report results of NIST projects of transitory or limited interest, including those that will be published subsequently in more comprehensive form. 


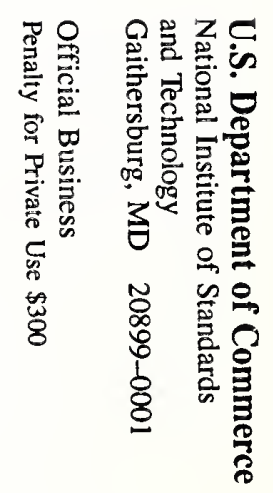

